\title{
Appropriate Use Criteria for PET Myocardial Perfusion Imaging
}

\author{
Thomas H. Schindler ${ }^{1}$, Timothy M. Bateman ${ }^{1}$, Daniel S. Berman ${ }^{1}$, Panithaya Chareonthaitawee ${ }^{1,2}$, Lorraine E. De \\ Blanche $^{3}$, Vasken Dilsizian ${ }^{1,2}$, Sharmila Dorbala ${ }^{1}$, Robert J. Gropler ${ }^{1}$, Leslee Shaw ${ }^{4,5}$, Prem Soman ${ }^{1,4}$, David E. Winchester ${ }^{6}$, \\ Hein Verberne ${ }^{7}$, Sukhjeet Ahuja ${ }^{1}$, Rob S. Beanlands ${ }^{1,2,4,8,9}$, Marcelo F. Di Carli ${ }^{1,10}$, Venkatesh L. Murthy ${ }^{1}$, \\ Terrence D. Ruddy ${ }^{1,8,9}$, and Ronald G. Schwartz ${ }^{1}$ \\ ${ }^{1}$ Society of Nuclear Medicine and Molecular Imaging, Reston, Virginia; ${ }^{2}$ American Society of Nuclear Cardiology, Fairfax, Virginia; \\ ${ }^{3}$ American College of Nuclear Medicine, Reston, Virginia; ${ }^{4}$ American College of Cardiology, Washington, D.C.; ${ }^{5}$ Society of \\ Cardiovascular Computed Tomography, Arlington, Virginia; ${ }^{6}$ American College of Physicians, Philadelphia, Pennsylvania; \\ ${ }^{7}$ European Association of Nuclear Medicine, Vienna, Austria; ${ }^{8}$ Canadian Society of Cardiovascular Nuclear and CT Imaging, Ottawa, \\ Ontario, Canada; ${ }^{9}$ Canadian Cardiovascular Society, Ottawa, Ontario, Canada; and ${ }^{10}$ American Heart Association, Dallas, Texas
}

\section{EXECUTIVE SUMMARY}

In the last decade, myocardial perfusion imaging (MPI) with PET has emerged to play a pivotal role in the clinical routine process for the detection of hemodynamically significant obstructive coronary artery disease (CAD) and cardiovascular risk stratification (1-5). The high spatial and contrast resolution in concert with photon attenuation-free images of PET have led to high image quality associated with the highest sensitivity and specificity of PET/CT perfusion imaging in the detection and characterization of CAD $(1,2,6,7)$. In addition, the noninvasive evaluation and quantification of global and regional myocardial blood flow (MBF) in milliliters per gram per minute during hyperemic stress and at rest, as well as the calculation of the resulting myocardial flow reserve (MFR), extends the scope of standard MPI from the detection of advanced and flow-limiting epicardial CAD to a comprehensive assessment of ischemic burden. This improved scope results not only from the traditionally sought significant left main or multivessel disease, but also from the more recently appreciated cardiac effects of nonobstructive CAD and coronary microvascular disease (CMD), which conveys important diagnostic and incremental prognostic information (1,2,4-11).

The increased availability and high sensitivity of PET MPI in concert with concerns about missed diagnoses, however, may at times lead to an inappropriate application of this technology. Thus, to avoid unnecessary financial burden on the health-care system and, in some cases, unnecessary exposure of patients to ionizing radiation, we have established a consensus document that outlines the most appropriate and cost-effective use of PET MPI. It is hoped that this expert guidance will help to render the use of PET MPI more consistent and will improve health-care outcomes for the targeted patient population while minimizing unnecessary imaging costs. The goal of this document is to describe the appropriate use of PET MPI in patients with suspected or known CAD and in patients with suspected microvascular angina. Through these recommendations, it is expected that PET MPI will be applied to benefit these patients in the most cost-effective manner.

Representatives from the Society of Nuclear Medicine and Molecular Imaging (SNMMI), the American College of Cardiology

Received Mar. 31, 2020; revision accepted Mar. 31, 2020. COPYRIGHT (c) 2020 by the Society of Nuclear Medicine and Molecular Imaging. DOI: 10.2967/jnumed.120.246280
(ACC), the American Society of Nuclear Cardiology (ASNC), the Canadian Cardiovascular Society (CCS), the Canadian Society of Cardiovascular Nuclear and CT Imaging (CSCNCTI), the Society of Cardiovascular CT (SCCT), the American Heart Association (AHA), the American College of Physicians (ACP), and the European Association of Nuclear Medicine (EANM) assembled as an autonomous workgroup to develop the following appropriate use criteria (AUC). This process was performed in accordance with the Protecting Access to Medicare Act of 2014. This legislation requires that all referring physicians consult AUC by using a clinical decision support mechanism before ordering any advanced diagnostic imaging services. Such services are defined as diagnostic MRI, CT, nuclear medicine procedures (including PET), and others as specified by the Secretary of Health and Human Services in consultation with physician specialty organizations and other stakeholders (12). The AUC in this paper are intended to aid referring medical practitioners in the appropriate use of PET MPI for the most common scenarios encountered in patients with suspected or known $\mathrm{CAD}$ as well as in patients with suspected microvascular angina.

\section{INTRODUCTION}

This document describes the appropriate use of MPI and concurrent MBF assessment with PET (PET MPI). The authors have strived to include the most common clinical scenarios for PET MPI in patients with suspected $\mathrm{CAD}$ or $\mathrm{CMD}$, or with known $\mathrm{CAD}$, in order to assist health-care practitioners in the cardiovascular field who are considering PET MPI. It is important to keep in mind, however, that a patient may have variations of the clinical scenarios covered in the current document, or signs or symptoms not described, for which PET MPI may still be indicated. Because each patient is unique, as is each patient's clinical presentation, this document is not intended to replace clinical judgment. PET MPI is also applied in conjunction with ${ }^{18}$ F-FDG PET for myocardial viability and cardiac sarcoidosis assessment, for which myocardial perfusion flow characterization is integral to diagnosis and, thus, patient management.

PET affords not only the assessment of relative differences in myocardial perfusion, but also, in conjunction with tracer kinetic modeling, the calculation of regional and global MBF of the left ventricle in absolute terms in milliliters per gram per minute $(1,2,6,7)$. This enables the calculation of MBF during pharmacologically induced hyperemic flow and at rest in milliliters per gram 
per minute. The assessment of hyperemic MBF and MFR (MFR = MBF during hyperemia/MBF at rest), therefore, allows the assessment of CMD as a potential substrate for angina symptoms or shortness of breath in the absence of obstructive CAD $(3,13)$. In this respect, the noninvasive evaluation and quantification of hyperemic MBF and MFR expand the scope of conventional MPI from detection of end-stage, advanced, flow-limiting epicardial CAD to early stages of atherosclerosis or CMD. It is important to keep in mind that the identification of stress-induced myocardial perfusion deficits with conventional MPI indicates hemodynamically obstructive CAD that carries important diagnostic and prognostic information for the evaluation of patients with suspected or known CAD. Conversely, the assessment of only the "relative" distribution of the radiotracer uptake in the left ventricular (LV) myocardium with standard MPI commonly identifies the culprit or most advanced CAD lesion, and the hemodynamic effects of less severe lesions may be missed in multivessel CAD disease $(2,8-10)$. Adding the concurrently PET-determined regional hyperemic MBF and MFR aids in the unraveling of the flow-limiting effects of the remaining CAD lesions of less or intermediate severity. Another advantage can also be seen in the identification of diffuse or balanced myocardial ischemia because of significant left main or 3-vessel disease with PET. Pronounced reductions in hyperemic MBF and MFR in all 3 major coronary artery distributions, in conjunction with transient ischemic cavity dilation at peak stress versus rest on PET imaging, identifies diffuse ischemia, otherwise likely to be missed by MPI alone $(2,8-$ 10). From a methodologic point of view, the high spatial and contrast resolution of the photon attenuation-free images of PET in conjunction with the superior properties of positron-emission blood flow radiotracers, such as ${ }^{13} \mathrm{~N}$-ammonia or ${ }^{82} \mathrm{Rb}$, affords several unique advantages of cardiac PET perfusion and flow assessment. The principle of coincidence detection, together with the superior properties of MBF radiotracers, has resulted in an increase in spatial and contrast resolution of PET MPI, explaining, at least in part, the high sensitivity (mean $\approx 92 \%$ ) and specificity (mean $\approx 90 \%$ ) in the detection of hemodynamically obstructive CAD lesions $(1,2)$.

A systematic review of the literature for this AUC document demonstrated that evidence was strongest for diagnostic accuracy and predictive utility of PET MPI perfusion defects in signifying hemodynamic effects of flow-limiting obstructive CAD. In addition, there was good evidence to support the diagnostic accuracy and predictive utility of abnormal MBF or flow quantification, as well as the predictive utility of reduced LV systolic function. Taken together, the evidence is excellent to good that findings on PET MPI perfusion and flow quantification precisely identify persons with $\mathrm{CAD}$ and, at the same time, find persons at risk for mortality and future cardiovascular events. Although the assessment of CMD in obstructive and nonobstructive $\mathrm{CAD}$ has incremental and independent prognostic value, further evidence is needed to clarify the diagnostic accuracy of PET for diagnosing CMD and the optimal threshold to define abnormal hyperemic MBF increases or MFR. Only a few studies describe the effects of PET MPI perfusion and flow quantification on the clinical decision-making process and clinical outcome (14-18), which thus warrants further evaluation in well-designed and large-scale clinical trials. In principle, further evidence is also needed to define the role of PET MPI perfusion and flow quantification in asymptomatic adults and children, leaving the decision of its use in these populations for the time being to best clinical judgment.
From the evidence of this systematic review, outcome data, clinical expertise, and standard clinical practice, we evaluated a total of 210 clinical scenarios for PET MPI and covered them in the following 11 sections: section 1: Symptomatic Patients with Suspected or Known CAD; section 2: Asymptomatic Patients (Without Symptoms or Ischemic Equivalent); section 3: Diagnosed Heart Failure (Resting LV Function Previously Assessed but No Prior CAD Evaluation); section 4: Evaluation of Patients with Known or Suspected Cardiac Sarcoidosis; section 5: Evaluation of Arrhythmias Without Ischemic Equivalent (No Prior Cardiac Evaluation); section 6: Syncope Without Ischemic Equivalent; section 7: Assessment of CMD in Symptomatic Patients; section 8: Specific Populations; section 9: Prior Testing or Procedures; section 10: Preoperative Evaluation for Noncardiac Surgery; and section 11: Determination of Exercise Level Before Initiation of Exercise Prescription or Cardiac Rehabilitation. This comprehensive list of elaborated clinical scenarios aims to guide health-care practitioners to an appropriate use of PET MPI, but the document does not claim to cover all potential clinical scenarios or to substitute for clinical judgment in an individual clinical scenario.

\section{METHODOLOGY}

\section{Expert Workgroup Selection}

The experts of this AUC workgroup were convened by the SNMMI to represent a multidisciplinary panel of health-care providers with substantive knowledge in the use of PET MPI. In addition to SNMMI members, representatives from the ACC, ASNC, CCS, CSCNCTI, SCCT, AHA, ACP, and EANM were included in the workgroup. Eighteen physician members were ultimately selected to participate and contribute to the AUC. A complete list of workgroup participants and external reviewers can be found in Appendix A. Appendix $B$ is a summary of definitions of terms and acronyms, Appendix $\mathrm{C}$ provides the disclosures and conflicts-of-interest statements, and Appendix D describes the solicitation of public commentary.

\section{AUC Development}

The process for AUC (11) development was modeled after the RAND/UCLA Appropriateness Method $(19,20)$ and included the development of a list of common indications for the use of PET in MPI, a systematic review of evidence related to these indications, and the development of an appropriateness score for each indication by using a modified Delphi process. This process strove to adhere to the standards of the Institute of Medicine of the National Academies for developing trustworthy clinical guidance (21). The process included a systematic synthesis of available evidence, individual and group ratings of the clinical indications by using a formal consensus process, and AUC recommendations based on final group ratings and discussions.

\section{Scope and Development of Clinical Scenarios}

To begin this process, the workgroup discussed various potential clinical scenarios for the appropriate use of MPI and concurrent MBF assessment with PET. For clinical scenarios, some of the relevant populations of interest were patients who had known or suspected CAD; who were obese; who were symptomatic; who had prior equivocal or discordant studies, suspected microvascular angina, prior revascularization, or failed medical therapy; and who were of all genders, ages, races, and geographic locations.

The workgroup identified 210 clinical scenarios for PET MPI that are evaluated and covered in 11 sections. The scenarios are intended to be as representative of the relevant patient population as possible 
for development of AUC. The resulting AUC are based on evidence and expert opinion regarding diagnostic accuracy and effects on clinical outcomes and clinical decision making as applied to each scenario. Other factors affecting the AUC recommendations were potential harm-including long-term harm that may be difficult to capture-costs, availability, and patient preferences.

\section{Systematic Review}

To inform the workgroup, a systematic review of the relevant evidence was commissioned by an independent group, the Pacific Northwest Evidence-Based Practice Center of Oregon Health and Science University (22). The primary purpose of the systematic review was to synthesize evidence on PET MPI for the detection of CAD and CMD, the prediction of future cardiovascular events, the effects on clinical decision making, and the effects on clinical outcomes in symptomatic adults and children. The workgroup selected the following key questions to guide the review:

1. In adults and children undergoing evaluation for symptoms of potential CAD, what is the accuracy of PET MPI for diagnosis of CAD or CMD?

2. In symptomatic adults and children, what is the utility of PET MPI for predicting future cardiovascular events?

3. In adults and children undergoing PET MPI for evaluation of symptoms of potential CAD, what are the effects on clinical decision making (use of treatments, subsequent testing)?

4. In adults and children undergoing PET MPI for evaluation of symptoms of potential CAD, what are the effects on clinical outcomes?

5. In adults without symptoms of CAD, what is the utility of PET MPI for predicting future cardiovascular events?

6. In asymptomatic adults who undergo PET MPI, what are the effects on clinical decision making (use of treatments, subsequent testing)?

Potential modifiers of effects of interest were demographic and clinical characteristics of the populations studied, use of stress techniques (pharmacologic or exercise), and use of alternative tracers. The inclusion and exclusion criteria for papers for this review were based on the study parameters established by the workgroup, using the PICOTS (population, intervention, comparisons, outcomes, timing, and setting) approach. Searches for relevant studies and systematic reviews were conducted in the following databases: Cochrane Central Register of Controlled Trials, Cochrane Database of Systematic Reviews, and Ovid MEDLINE (through November 2017). These searches were supplemented by reviewing the reference lists of relevant publications and suggestions from SNMMI workgroup members.

Two investigators independently reviewed abstracts and fulltext articles against prespecified eligibility criteria, as defined by PICOTS. For diagnostic accuracy, we included studies of symptomatic adults and children undergoing imaging with PET MPI or PET/CT MPI with any tracer for suspicion of or confirmation of CAD or CMD. Studies had to assess diagnostic accuracy against a reference standard of coronary angiography, usually a $>50 \%$ or $>70 \%$ reduction in luminal diameter for an anatomic diagnosis of $\mathrm{CAD}$, with or without a functional measure of obstruction (usually a fractional flow reserve of $<0.8$ ). The reference standard in the only study that assessed CMD was epicardial artery vasomotor dysfunction during cold pressor testing on coronary angiography; in the 2 studies that assessed cardiac allograft vasculopathy (CAV), it was the International Society for Heart and Lung Transplantation criteria, including angiographic stenosis and evidence of allograft dysfunction. For predictive utility, we included longitudinal studies of symptomatic and asymptomatic adults and children regarding the association between PET MPI findings and death and future cardiovascular events, as well as clinical decision making (use of treatments, subsequent testing) and clinical outcomes (e.g., mortality, morbidity, quality of life, and harms). PET findings included perfusion deficits at rest or with stress and measures of MBF, including response to cold pressor testing. For predictive utility, we also evaluated PET measures of left ventricular ejection fraction (LVEF). Non-English language articles and studies published only as conference abstracts were excluded.

Two investigators independently assessed the quality (risk of bias) of each study as "good," "fair," or "poor" by using predefined criteria that were specific for each study design. Specifically, AMSTAR (A MeaSurement Tool to Assess systematic Reviews) (23) was used for systematic reviews (except diagnostic accuracy), adapted by the US Preventive Services Task Force criteria for randomized trials and cohort studies, and the QUADAS-2 (Quality Assessment of Diagnostic Accuracy Studies-2) (24) for primary studies and systematic reviews of diagnostic accuracy. Discrepancies were resolved through a consensus process. The strength of overall evidence was graded as high, moderate, low, or very low by using GRADE (Grading of Recommendations Assessment, Development and Evaluation) methods on the basis of quality of evidence, consistency, directness, precision, and reporting bias.

Database searches resulted in 1,189 potentially relevant articles. After a dual review of abstracts and titles, we selected 206 articles for full-text dual review, and 76 studies (in 82 publications) were determined to meet inclusion criteria and were included in this review.

\section{Rating and Scoring}

In developing these AUC (11) for PET MPI, the workgroup members used the following definition of appropriateness to guide their considerations and group discussions: "The concept of appropriateness, as applied to health care, balances risk and benefit of a treatment, test, or procedure in the context of available resources for an individual patient with specific characteristics" (25).

At the beginning of the process, workgroup members convened via webinar/teleconference to develop the initial clinical indications. On evaluating the evidence summary of the systematic literature review, the workgroup further refined its draft clinical indications to ensure their accuracy and facilitate consistent interpretation when scoring each indication for appropriateness. Using the evidence summary, workgroup members were first asked individually to assess the appropriateness and provide a score for each identified indication. Workgroup members then convened in a group setting for several successive webinars to discuss each indication and associated scores from the first round of individual scoring. After deliberate discussion, a consensus score was determined and then assigned to the associated appropriate use indication. For this scoring round, the expert panel was encouraged to include their clinical expertise in addition to the available evidence in determining the final scores. All members contributed to the final discussion, and no one was forced into consensus. After the rating process was completed, the final appropriate use ratings were summarized in a format similar to that outlined by the RAND/UCLA Appropriateness Method.

The workgroup scored each indication as "appropriate," "may be appropriate," or "rarely appropriate" on a scale from 1 to 9 . Scores 7-9 indicate that the use of the procedure is appropriate for 
the specific clinical indication and is generally considered acceptable. Scores 4-6 indicate that the use of the procedure may be appropriate for the specific indication. This implies that more research is needed to classify the indication definitively. Scores 1-3 indicate that the use of the procedure is rarely appropriate for the specific indication and is generally not considered acceptable.

As stated by other societies that develop AUC, the division of these scores into 3 general levels of appropriateness is partially arbitrary, and the numeric designations should be viewed as a continuum. In addition, if there was a difference in clinical opinion for an indication such that workgroup members could not agree on a common score, that indication was given a "may be appropriate" rating to indicate a lack of agreement on appropriateness based on the available literature and the members' collective clinical opinion, indicating the need for additional research.

\section{SECTION 1: SYMPTOMATIC PATIENTS WITH SUSPECTED OR KNOWN CAD}

\section{Introduction and Background}

The accuracy and prognostic value of MPI (PET or SPECT) to define ischemia in order to detect CAD and to risk stratify patients is well established. A wealth of data for SPECT and now a large body of evidence for PET MPI support this concept, applying PET for diagnosis and prognostication. Driven by the Bayes' theorem, defining the pretest likelihood of disease must be a step in considering a decision to order PET MPI. This, along with knowing the impact of a positive or negative test result on decision making, helps the clinician and imager determine the need for PET MPI. Guidelines indicate a role for ischemia testing with MPI (SPECT and PET can both provide this role). However, the superiority of PET over SPECT for accuracy is now well established, as is the prognostic value of PET MPI. Although not explicitly mentioned in the case scenarios, the advantage of PET for flow quantification is implicit when considering PET for symptomatic patients, given its added value in the diagnosis of obstructive disease, in particular multivessel CAD, and in the detection of CMD or even nonresponsiveness to pharmacologic stress. Moreover, the incremental prognostic value of flow quantification beyond relative MPI further empowers PET as a risk assessment tool beyond the known utility of relative perfusion imaging alone.

\section{Clinical Scenarios and AUC Scores}

Table 1 presents the following clinical scenarios and final AUC scores for the use of stress-rest perfusion PET to assess the presence of flow-limiting obstructive CAD as a potential substrate for chest pain (or equivalent) symptoms, or for risk stratification and prognostic value, in symptomatic patients with suspected or known CAD.

Clinical Scenarios 1-15. The following scenarios address patients presenting a low pretest probability of CAD:

Scenario 1: Low pretest probability for CAD, with an interpretable electrocardiogram (ECG) and where the patient is able to undergo adequate exercise stress (Score 2 - Rarely Appropriate)

Scenario 2: Low pretest probability of CAD, ECG uninterpretable or unable to exercise (Score 3 - Rarely Appropriate)

Scenario 3: Low pretest probability for CAD, with an uninterpretable ECG (Score 3 - Rarely Appropriate)

Scenario 4: Low pretest probability for CAD, where the patient is unable to undergo adequate exercise stress (Score 3 - Rarely Appropriate)
The following scenarios address patients presenting an intermediate pretest probability of CAD:

Scenario 5: In symptomatic patients with intermediate pretest probability for $\mathrm{CAD}$, where ECG is interpretable and where the patient is able to undergo adequate exercise stress (Score 7 - Appropriate)

Scenario 6: Intermediate pretest probability for CAD, where ECG is uninterpretable (Score 9 - Appropriate)

Scenario 7: Intermediate pretest probability for CAD, where the patient is unable to undergo adequate exercise stress (Score 9 - Appropriate)

The following scenarios address patients presenting a high pretest probability of CAD:

Scenario 8: In symptomatic patients with high pretest probability for CAD, where ECG is interpretable and where the patient is able to undergo adequate exercise stress (Score 8 - Appropriate)

Scenario 9: High pretest probability for CAD, where ECG is uninterpretable (Score 9 - Appropriate)

Scenario 10: High pretest probability for CAD, where the patient is unable to undergo adequate exercise stress (Score $9-$ Appropriate)

The following scenarios address symptomatic patients presenting to the emergency department (ED) (or inpatients) with acute coronary syndrome (ACS) features:

Scenario 11: Patients presenting to the ED (or inpatients) with symptoms suspicious for CAD without (or with) known CAD who have features consistent with non-ST elevation myocardial infarction (non-STEMI)/ACS (increase in troponin (Tn) level(s) or dynamic ECG changes) (Score 1 - Rarely Appropriate)

Scenario 12: Patients presenting to the ED (or inpatients) with symptoms suspicious for CAD without (or with) known CAD who have features consistent with unstable angina/ACS (no increase in Tn level(s), no dynamic ECG changes) (Score 7 - Appropriate)

The following scenario addresses patients presenting to the ED (or inpatients) with chest pain or equivalent symptoms without (or with) known CAD who have equivocal Tn level(s) or a temporal Tn pattern not consistent with ACS:

Scenario 13: Patients presenting to the ED (or inpatients) with chest pain or equivalent symptoms without (or with) known CAD who have equivocal Tn level(s) or a temporal Tn pattern not consistent with ACS (Score 8 - Appropriate)

The following scenario addresses patients presenting to the ED (or inpatients) with symptoms of chest pain that may be due to ACS, with normal Tn level(s) and no dynamic ECG changes:

Scenario 14: Patients presenting to the ED (or inpatients) with symptoms of chest pain that may be due to ACS, normal Tn level(s) and no dynamic ECG changes (Score 8 - Appropriate)

The following scenario addresses patients presenting to the ED (or inpatients) with symptoms of chest pain that is unlikely to be due to ACS, with normal Tn level(s) and no dynamic ECG changes:

Scenario 15: Patients presenting to the ED (or inpatients) with symptoms of chest pain that is unlikely to be due to ACS, normal Tn level(s) and no dynamic ECG changes (Score 7 - Appropriate) 
TABLE 1

Clinical Scenarios for the Use of Stress-Rest Perfusion PET to Assess the Presence of Flow-Limiting Obstructive $\mathrm{CAD}$ as a Potential Substrate for Chest Pain (or Equivalent) Symptoms, or for Risk Stratification and Prognostic Value, in Symptomatic Patients with Suspected or Known CAD

\begin{tabular}{|c|c|c|c|}
\hline Scenario no. & Description & Appropriateness & Score \\
\hline \multicolumn{4}{|c|}{ Symptomatic patients with low pretest probability of CAD: interpretable ECG AND able to exercise } \\
\hline 1 & $\begin{array}{l}\text { Symptomatic patients with a low pretest probability for CAD, who have an interpretable } \\
\text { ECG and are able to undergo adequate exercise stress }\end{array}$ & $\begin{array}{l}\text { Rarely } \\
\text { Appropriate }\end{array}$ & 2 \\
\hline \multicolumn{4}{|c|}{ Symptomatic patients with low pretest probability of CAD: uninterpretable ECG OR unable to exercise } \\
\hline 2 & $\begin{array}{l}\text { Symptomatic patients with a low pretest probability of CAD, who have an uninterpretable } \\
\text { ECG or are unable to exercise }\end{array}$ & $\begin{array}{l}\text { Rarely } \\
\text { Appropriate }\end{array}$ & 3 \\
\hline 3 & $\begin{array}{l}\text { Symptomatic patients with a low pretest probability for CAD, who have an uninterpretable } \\
\text { ECG }\end{array}$ & $\begin{array}{l}\text { Rarely } \\
\text { Appropriate }\end{array}$ & 3 \\
\hline 4 & $\begin{array}{l}\text { Symptomatic patients with a low pretest probability for CAD, who are unable to undergo } \\
\text { adequate exercise stress }\end{array}$ & $\begin{array}{l}\text { Rarely } \\
\text { Appropriate }\end{array}$ & 3 \\
\hline \multicolumn{4}{|c|}{ Symptomatic patients with intermediate pretest probability of CAD: interpretable ECG AND able to exercise } \\
\hline 5 & $\begin{array}{l}\text { Symptomatic patients with an intermediate pretest probability for CAD, who have an } \\
\text { interpretable ECG and are able to undergo adequate exercise stress }\end{array}$ & Appropriate & 7 \\
\hline \multicolumn{4}{|c|}{ Symptomatic patients with intermediate pretest probability of CAD: uninterpretable ECG OR unable to exercise } \\
\hline 6 & $\begin{array}{l}\text { Symptomatic patients with an intermediate pretest probability for CAD, who have an } \\
\text { uninterpretable ECG }\end{array}$ & Appropriate & 9 \\
\hline 7 & $\begin{array}{l}\text { Symptomatic patients with an intermediate pretest probability for CAD, who are unable } \\
\text { to undergo adequate exercise stress }\end{array}$ & Appropriate & 9 \\
\hline \multicolumn{4}{|c|}{ Symptomatic patients with high pretest probability of CAD: interpretable ECG AND able to exercise } \\
\hline 8 & $\begin{array}{l}\text { Symptomatic patients with a high pretest probability for CAD, who have an interpretable } \\
\text { ECG and are able to undergo adequate exercise stress }\end{array}$ & Appropriate & 8 \\
\hline \multicolumn{4}{|c|}{ Symptomatic patients with high pretest probability of CAD: uninterpretable ECG OR unable to exercise } \\
\hline 9 & $\begin{array}{l}\text { Symptomatic patients with a high pretest probability for CAD, who have an uninterpretable } \\
\text { ECG }\end{array}$ & Appropriate & 9 \\
\hline 10 & $\begin{array}{l}\text { Symptomatic patients with a high pretest probability for CAD, who are unable to undergo } \\
\text { adequate exercise stress }\end{array}$ & Appropriate & 9 \\
\hline \multicolumn{4}{|c|}{ Patients who present to the ED (or inpatients) with ACS features } \\
\hline 11 & $\begin{array}{l}\text { Patients who present to the ED (or inpatients) with symptoms suspicious for CAD with or } \\
\text { without known CAD, who have features consistent with non-STEMI or ACS (increase in } \\
\text { Tn levels or dynamic ECG changes) }\end{array}$ & $\begin{array}{l}\text { Rarely } \\
\text { Appropriate }\end{array}$ & 1 \\
\hline 12 & $\begin{array}{l}\text { Patients who present to the ED (or inpatients) with symptoms suspicious for CAD with or } \\
\text { without known CAD, who have features consistent with unstable angina or ACS (but no } \\
\text { increase in Tn levels and no dynamic ECG changes) }\end{array}$ & Appropriate & 7 \\
\hline \multicolumn{4}{|c|}{$\begin{array}{l}\text { Patients who present to the ED (or inpatients) with chest pain or equivalent symptoms with or without known CAD, who have } \\
\text { equivocal Tn levels or a temporal Tn pattern not consistent with ACS }\end{array}$} \\
\hline 13 & $\begin{array}{l}\text { Patients who present to the ED (or inpatients) with chest pain or equivalent symptoms with } \\
\text { or without known CAD, who have equivocal Tn levels or a temporal Tn pattern not } \\
\text { consistent } \\
\text { with ACS }\end{array}$ & Appropriate & 8 \\
\hline \multicolumn{4}{|c|}{$\begin{array}{l}\text { Patients who present to the ED (or inpatients) with symptoms of chest pain that may be due to ACS, normal Tn levels, and no } \\
\text { dynamic ECG changes }\end{array}$} \\
\hline 14 & $\begin{array}{l}\text { Patients who present to the ED (or inpatients) with symptoms of chest pain that may be } \\
\text { due to ACS, with normal Tn levels and no dynamic ECG changes }\end{array}$ & Appropriate & 8 \\
\hline \multicolumn{4}{|c|}{$\begin{array}{l}\text { Patients who present to ED (or inpatients) with symptoms of chest pain that are unlikely to be due to ACS, normal Tn levels, } \\
\text { and no dynamic ECG changes }\end{array}$} \\
\hline 15 & $\begin{array}{l}\text { Patients who present to the ED (or inpatients) with symptoms of chest pain that are unlikely } \\
\text { to be due to ACS, with normal Tn levels and no dynamic ECG changes }\end{array}$ & Appropriate & 7 \\
\hline
\end{tabular}

The preceding scenarios evaluate the role of MPI PET in the management of symptomatic patients with suspected or known CAD (scenarios 1-10) or who present to the ED (scenarios 11-15).
In symptomatic patients with suspected or known CAD, scintigraphic MPI such as with PET (PET MPI) is best performed for risk stratification with a clinically intermediate risk of subsequent 
cardiac events $(1,4,5,26-28)$. This also relates to the optimal application of PET MPI to patients with an intermediate likelihood of having CAD. Critical determinants of outcome in patients with CAD can be determined by stress-related myocardial perfusion and function $(26,27,29,30)$. Although indices that determine the extent of LV dysfunction (LVEF, the extent of infarcted myocardium, transient ischemic dilation of the left ventricle, and increased radiotracer uptake in the lungs) are relevant predictors of cardiac mortality, parameters related to ischemia induction (exertional symptoms, ECG alterations, extent and severity of reversible perfusion deficits, and ventricular wall motion abnormalities) are independent predictors of acute ischemic syndromes $(26,28-32)$. PET MPI, in particular, affords the advantage of high diagnostic accuracy, concurrent evaluation of myocardial perfusion (radiotracer uptake) and regional MFR, and wall motion assessment at peak pharmacologic stress $(2,8,10,26,33-39)$. This enables the accurate identification and description of the extent and severity of stress-induced ischemia in individuals with suspected CAD and intermediate and high pretest probability. In addition, the concurrent assessment of regional and global hyperemic MBF and MFR enables the detection and characterization of flow-limiting effects of each single vessel in multivessel disease $(2,4-8,10,11,40-46)$, and reductions in global MFR also convey important and incremental prognostic information in patients with and without obstructive CAD (2,3,7,14,26,39,46-48).

In symptomatic patients with suspected CAD but low pretest probability in scenarios $1-4$, the application of PET MPI in general is rarely appropriate after standard clinical practice guidelines $(27,28)$. Conversely, for scenarios 5-7 in the category of intermediate pretest probability for CAD, the performance of PET MPI has been judged as appropriate in accordance with standard clinical practice in scintigraphic MPI $(27,28)$. In patients with high pretest probability for CAD, who have an uninterpretable ECG and are able or unable to exercise, PET MPI was seen as appropriate (scenarios $8-10)$ according to standard clinical practice in MPI $(27,28)$.

In symptomatic patients who present to the ED (or inpatients) with ACS features that are consistent with non-STEMI or ACS (increase in Tn levels or dynamic ECG changes) (scenario 11), MPI PET is not indicated, as these patients are at high risk and, thus, they are commonly referred to further triage with invasive coronary angiography and potential intervention according to standard clinical practice $(49,50)$. In scenario 12 , this group of patients with some type of unstable angina or ACS and with positive Tn results but with neither an increase in Tn levels later nor dynamic ECG changes, PET MPI was seen as appropriate for further cardiovascular risk stratification and triage of the patient $(27,28,32,49-54)$.

Scenarios 13-15 address the role of PET MPI in patients who present to the ED (or inpatients) with chest pain or equivalent symptoms with or without known CAD who have equivocal Tn levels or a temporal Tn pattern not consistent with ACS (scenario 13); patients who present to the ED (or inpatients) with symptoms of chest pain that may be due to ACS, with normal Tn levels and no dynamic ECG changes (scenario 14); and patients who present to the ED (or inpatients) with symptoms of chest pain that are unlikely to be due to ACS, with normal Tn levels and no dynamic ECG changes (scenario 15). Given the clinical constellation and standard clinical practice $(27,28,32,49-54)$, these scenarios were seen as appropriate.

\section{Summary of Recommendations}

Rest-stress PET MPI is considered an appropriate test in patients with or without known CAD who have symptoms with an intermediate-to-high pretest likelihood of disease regardless of whether the patient has normal ECG results or can (or cannot) exercise. Both diagnostic accuracy and prognostic value are considerations. PET MPI is not appropriate when patients have a low pretest likelihood of disease. For patients in the ED or for inpatients with chest pain or symptoms related potentially to ischemia, when there are clear markers or high risk (elevated Tn levels, dynamic ECG changes), PET MPI is rarely appropriate. On the other hand, when such markers are not present, PET MPI is generally considered appropriate.

\section{SECTION 2: ASYMPTOMATIC PATIENTS (WITHOUT SYMPTOMS OR ISCHEMIC EQUIVALENT)}

\section{Background}

Confirmation of the effectiveness of systematic screening for CAD in asymptomatic patients has been elusive. The evidence to support the use of stress testing and imaging in general and PET MPI in particular is limited. The guidelines suggest that only highrisk subjects should be considered for further noninvasive or invasive testing. The clinical cardiovascular risk evaluation is commonly based on the patient's 10-y atherosclerotic cardiovascular disease (ASCVD) risk estimate according to the ACC/AHA guidelines (low ASCVD risk $<7.5 \%$, intermediate ASCVD risk $\geq 7.5 \%-20 \%$, high ASCVD risk $>20 \%$ ) (55).

Although the data are limited on how to manage asymptomatic patients who have a positive test result, the principles of risk stratification used in other patient groups described in this document also apply to asymptomatic individuals. The scenarios identified below represent common clinical situations in which imaging tests are often considered. In all of these categories, the use of quantitative $\mathrm{MBF}$ and flow reserve in combination with semiquantitative PET MPI has generally been considered to be a unique advantage of PET MPI over SPECT MPI. The combination of PET MPI and flow quantification can help improve the sensitivity for detecting obstructive $\mathrm{CAD}$ or $\mathrm{CMD}$ and refine the cardiovascular risk assessment.

\section{Clinical Scenarios and AUC Scores}

Table 2 presents the following clinical scenarios and final AUC scores for the use of PET MPI in asymptomatic patients (without symptoms or ischemic equivalent).

Clinical Scenarios 16-25: Asymptomatic Patients (Without Symptoms or Ischemic Equivalent) by Pretest CAD Risk.

Scenario 16: Asymptomatic patients with a low ASCVD risk $(<7.5 \%)$ (Score 1 - Rarely Appropriate)

Scenario 17: Asymptomatic patients with an intermediate ASCVD risk (7.5\%-20\%), who have an interpretable resting ECG and are able to exercise (Score 2 - Rarely Appropriate)

Scenario 18: Asymptomatic patients with an intermediate ASCVD risk $(7.5 \%-20 \%)$, who have an uninterpretable resting ECG or are unable to exercise (Score 5 - May be Appropriate)

Scenario 19: Asymptomatic patients with a high ASCVD risk $(>20 \%)$ (Score 5 - May be Appropriate)

Scenario 20: Asymptomatic patients with an intermediate ASCVD risk (7.5\%-20\%), who have a calcium score of 400-1,000 (Score 6 - May be Appropriate)

Scenario 21: Asymptomatic patients with a high ASCVD risk $(>20 \%$ ), who have a calcium score of 400-1,000 (Score 8 - Appropriate) 
TABLE 2

Clinical Scenarios for the Use of PET MPI in Asymptomatic Patients (Without Symptoms or Ischemic Equivalent)

\begin{tabular}{|c|c|c|c|}
\hline Scenario no. & Description & Appropriateness & Score \\
\hline \multicolumn{4}{|c|}{ Asymptomatic patients by pretest CAD risk } \\
\hline 16 & Asymptomatic patients with a low pretest global CAD risk $(<7.5 \%)$ & Rarely Appropriate & 1 \\
\hline 17 & $\begin{array}{l}\text { Asymptomatic patients with an intermediate pretest global CAD risk }(7.5 \%-20 \%) \text {, } \\
\text { who have an interpretable resting ECG and are able to exercise }\end{array}$ & Rarely Appropriate & 2 \\
\hline 18 & $\begin{array}{l}\text { Asymptomatic patients with an intermediate pretest global CAD risk }(7.5 \%-20 \%) \text {, } \\
\text { who have an uninterpretable resting ECG or are unable to exercise }\end{array}$ & May be Appropriate & 5 \\
\hline 19 & Asymptomatic patients with a high pretest probability of CAD $(>20 \%)$ & May be Appropriate & 5 \\
\hline 20 & $\begin{array}{l}\text { Asymptomatic patient with an intermediate pretest CAD risk }(7.5 \%-20 \%) \text {, who } \\
\text { have a calcium score of } 400-1,000\end{array}$ & May be Appropriate & 6 \\
\hline 21 & $\begin{array}{l}\text { Asymptomatic patients with a high pretest CAD risk ( }>20 \%) \text {, who have a calcium } \\
\text { score of } 400-1,000\end{array}$ & Appropriate & 8 \\
\hline 22 & Asymptomatic patients who have a calcium score of $>1,000$ & Appropriate & 8 \\
\hline 23 & Asymptomatic patients with peripheral vascular disease & May be Appropriate & 5 \\
\hline 24 & Asymptomatic patients with a family history of premature CAD & Rarely Appropriate & 3 \\
\hline 25 & Asymptomatic patients with familial hyperlipidemia & May be Appropriate & 5 \\
\hline \multicolumn{4}{|c|}{ Asymptomatic patients with equivocal or abnormal prior test results } \\
\hline 26 & $\begin{array}{l}\text { Asymptomatic patients with equivocal or abnormal prior test results from CCTA } \\
\text { or ICA }\end{array}$ & Appropriate & 8 \\
\hline 27 & $\begin{array}{l}\text { Asymptomatic patients with recent }(<90 \mathrm{~d} \text { ) equivocal or abnormal prior test } \\
\text { results from stress testing (imaging or nonimaging) }\end{array}$ & May be Appropriate & 6 \\
\hline 28 & Asymptomatic patients with regional or global LV systolic dysfunction & Appropriate & 8 \\
\hline 29 & Asymptomatic patients with new LBBB & Appropriate & 8 \\
\hline 30 & Asymptomatic patients with AF & May be Appropriate & 5 \\
\hline 31 & $\begin{array}{l}\text { Asymptomatic patients with abnormal resting ECG results that show abnormal } \\
\text { or pathologic } Q \text { waves }\end{array}$ & May be Appropriate & 6 \\
\hline 32 & $\begin{array}{l}\text { Asymptomatic patients with abnormal resting ECG results that show ST-T } \\
\text { segment abnormalities }\end{array}$ & May be Appropriate & 5 \\
\hline 33 & Asymptomatic patients with known stable CAD without prior revascularization & May be Appropriate & 6 \\
\hline \multicolumn{4}{|c|}{ Asymptomatic patients with a history of revascularization (PCI or CABG) } \\
\hline 34 & Asymptomatic patients with a history of $\mathrm{PCl}$ of $<2 \mathrm{y}$ & Rarely Appropriate & 2 \\
\hline 35 & Asymptomatic patients with a history of $\mathrm{PCl}$ of $>2 \mathrm{y}$ & May be Appropriate & 6 \\
\hline 36 & Asymptomatic patients with a prior CABG of $<5 \mathrm{y}$ & Rarely Appropriate & 2 \\
\hline 37 & Asymptomatic patients with a prior CABG of $>5 \mathrm{y}$ & May be Appropriate & 6 \\
\hline \multicolumn{4}{|c|}{ Asymptomatic patients with prior heart transplantation } \\
\hline 38 & Evaluation for CAV in asymptomatic patients with prior heart transplantation & Appropriate & 8 \\
\hline \multicolumn{4}{|c|}{ Asymptomatic patients being considered for solid organ transplantation (kidney, lung, liver) } \\
\hline 39 & $\begin{array}{l}\text { Evaluation for CAD in patients being considered for solid organ transplantation } \\
\text { (e.g., kidney, lung, liver) }\end{array}$ & Appropriate & 8 \\
\hline \multicolumn{4}{|c|}{ Asymptomatic patients undergoing cancer treatment } \\
\hline 40 & $\begin{array}{l}\text { Asymptomatic patients during or after chemotherapy or radiation therapy } \\
\text { for cancer who have a reduced LVEF }\end{array}$ & May be Appropriate & 5 \\
\hline 41 & $\begin{array}{l}\text { Asymptomatic patients during or after chemotherapy or radiation therapy } \\
\text { for cancer }\end{array}$ & Rarely Appropriate & 1 \\
\hline \multicolumn{4}{|c|}{ Asymptomatic patients with a history of coronary vasculitis or high-risk coronary anomalies } \\
\hline 42 & $\begin{array}{l}\text { Asymptomatic patients with a history of coronary vasculitis and evidence of } \\
\text { structurally abnormal coronary arteries (e.g., aneurysms) }\end{array}$ & Appropriate & 7 \\
\hline 43 & Asymptomatic patients with a history of high-risk coronary anomalies & May be Appropriate & 5 \\
\hline
\end{tabular}


Scenario 22: Asymptomatic patients who have a calcium score of $>1,000$ (Score 8 - Appropriate)

Scenario 23: Asymptomatic patients with peripheral vascular disease (Score 5 - May be Appropriate)

Scenario 24: Asymptomatic patients with a family history of premature CAD (Score 3 - Rarely Appropriate)

Scenario 25: Asymptomatic patients with familial hyperlipidemia (Score 5 - May be Appropriate)

PET was rated as rarely appropriate in asymptomatic patients with a low $(<7.5 \%)$ or intermediate ASCVD risk $(7.5 \%-20 \%)$ if they have an interpretable resting ECG (e.g., left bundle branch block [LBBB], paced rhythm, Wolff-Parkinson-White changes) and are able to exercise (scenarios 16 and 17). In keeping with other modalities, the use of PET was rated as may be appropriate in patients with an intermediate ASCVD risk who have an uninterpretable resting ECG or who are unable to exercise, as well as in those with a high ASCVD risk (>20\%) (scenarios 18 and 19). Regarding asymptomatic patients with prior coronary artery calcium (CAC) scoring, the use of PET was rated as may be appropriate in patients with an intermediate ASCVD risk and a CAC score of 400-1,000 (scenario 20). However, PET MPI was rated as appropriate in patients with a high ASCVD risk $(>20 \%)$ and a CAC score of $400-1,000$ or a very high CAC score $(>1,000)$ regardless of clinical risk (scenarios 21 and 22). This rating was based on the fact that the presence of an elevated CAC score $(>400)$ increases the likelihood of obstructive CAD and risk of events, especially in patients with multiple coronary risk factors with a high ASCVD risk. The use of PET in patients with a history of peripheral vascular disease (e.g., stroke) or familial hyperlipidemia was rated as may be appropriate because their clinical ASCVD risk is high (scenarios 23 and 25). However, a family history of premature CAD without any other risk qualifier was rated as rarely appropriate (scenario 24) (28,56-66).

Clinical Scenarios 26-33: Asymptomatic Patients with Equivocal or Abnormal Prior Test Results.

Scenario 26: Asymptomatic patients with equivocal or abnormal prior test results from coronary CT angiography (CCTA) or invasive coronary angiography (ICA) (Score 8 - Appropriate)

Scenario 27: Asymptomatic patients with recent $(<90$ d) equivocal or abnormal prior test results from stress testing (imaging or nonimaging) (Score 6 - May be Appropriate)

Scenario 28: Asymptomatic patients with regional or global LV systolic dysfunction (Score 8 - Appropriate)

Scenario 29: Asymptomatic patients with new LBBB (Score 8 Appropriate)

Scenario 30: Asymptomatic patients with atrial fibrillation (AF) (Score 5 - May be Appropriate)

Scenario 31: Asymptomatic patients with abnormal resting ECG results that show abnormal or pathologic Q waves (Score 6 - May be Appropriate)

Scenario 32: Asymptomatic patients with abnormal resting ECG results that show ST-T segment abnormalities (Score 5 - May be Appropriate)

Scenario 33: Asymptomatic patients with known stable CAD without prior revascularization (Score 6 - May be Appropriate)
The use of PET was considered appropriate in patients with equivocal or abnormal coronary angiography (CCTA or ICA) results, regional or global LV dysfunction, or new LBBB (scenarios 26, 28 , and 29). PET imaging was rated as may be appropriate in asymptomatic patients with recent $(<90 \mathrm{~d})$ abnormal or equivocal stress testing with or without imaging, as well as in those with atrial fibrillation (AF), abnormal resting ECG results showing pathologic Q waves or ST-T segment abnormalities, or stable CAD without prior revascularization (scenarios 27, 30-33) (28,67-71).

Clinical Scenarios 34-37: Asymptomatic Patients with Prior Revascularization (Percutaneous Coronary Intervention [PCI] or Coronary Artery Bypass Graft [CABG]). The following scenarios address asymptomatic patients with a history of PCI:

Scenario 34: Asymptomatic patients with a history of PCI of $<2$ y (Score 2 - Rarely Appropriate)

Scenario 35: Asymptomatic patients with a history of PCI of $>2$ y (Score 6 - May be Appropriate)

The following scenarios address asymptomatic patients with a prior CABG.

Scenario 36: Asymptomatic patients with a prior CABG of $<5 \mathrm{y}$ (Score 2 - Rarely Appropriate)

Scenario 37: Asymptomatic patients with a prior CABG of $>5 \mathrm{y}$ (Score 6 - May be Appropriate)

In keeping with the prior multimodality AUC document, the use of PET in asymptomatic patients with a history of prior PCI $(<2$ y) or CABG $(<5$ y) was considered rarely appropriate. However, PET MPI was rated as may be appropriate for asymptomatic patients $>2$ y after PCI or $>5$ y after CABG (scenarios 34-37) $(28,61)$.

Clinical Scenario 38: Asymptomatic Patients with Prior Heart Transplantation.

Scenario 38: Evaluation for CAV in asymptomatic patients with prior heart transplantation (Score 8 - Appropriate)

Annual surveillance to assess the presence and severity of CAV is the current standard of care after orthotopic heart transplantation. Although the current standard for CAV screening is ICA, it is considered inadequate because of its low yield and limited sensitivity to detect diffuse CAV. In addition, it is a costly, invasive procedure with a low but measurable risk for complications that is associated with relative contraindications in many patients transferred from outside the hospital because of the high prevalence of renal dysfunction. Noninvasive imaging modalities such as dobutamine stress echocardiography and SPECT MPI are relatively insensitive for the detection of CAV. Recent evidence supports a unique role for quantitative PET imaging for CAV screening with high accuracy. In keeping with this evidence, the workgroup rated the use of PET MPI for CAV screening as appropriate (scenario 38) (68,72-75).

Clinical Scenario 39: Asymptomatic Patients Being Considered for Solid Organ Transplantation (Kidney, Lung, Liver).

Scenario 39: Evaluation for CAD in patients being considered for solid organ transplantation (e.g., kidney, lung, liver) (Score 8 - Appropriate)

Although coronary revascularization before noncardiac surgery has not been shown to reduce the risk of cardiac events, preoperative noninvasive screening for obstructive CAD is commonly 
performed among patients who are candidates for solid organ transplantation. The rationale for such testing in these candidates includes the need to determine the perioperative risk and whether the patient's cardiovascular risk is high enough that organ transplantation would be futile and lead to an inappropriate use of a scarce organ. Given the high sensitivity of quantitative PET MPI for detection of obstructive $\mathrm{CAD}$, the workgroup rated its use in this group of patients as appropriate (scenario 39) (76).

Clinical Scenarios 40 and 41: Asymptomatic Patients Undergoing Cancer Treatment.

Scenario 40: Asymptomatic patients during or after chemotherapy or radiation therapy for cancer who have a reduced LVEF (Score 5 - May be Appropriate)

Scenario 41: Asymptomatic patients during or after chemotherapy or radiation therapy for cancer (Score 1 - Rarely Appropriate)

The use of PET MPI in patients with cancer before or during chemotherapy or radiation therapy was rated as may be appropriate in asymptomatic patients with new LV dysfunction (scenario 40). This was predicated on the fact that radiotherapy and newer chemotherapeutic drugs may cause accelerated progression of atherosclerosis. However, its use in patients with normal LV function was rated as rarely appropriate (scenario 41) (68).

Clinical Scenarios 42 and 43: Asymptomatic Patients with a History of Coronary Vasculitis or High-Risk Coronary Anomalies.

Scenario 42: Asymptomatic patients with a history of coronary vasculitis and evidence of structurally abnormal coronary arteries (e.g., aneurysms) (Score 7 - Appropriate)

Scenario 43: Asymptomatic patients with a history of high-risk coronary anomalies (Score 5 - May be Appropriate)

The use of PET MPI in asymptomatic patients with a history of coronary vasculitis (e.g., Kawasaki disease) and evidence of structurally abnormal coronary arteries (e.g., coronary obstruction or aneurysms) was rated as appropriate (scenario 42). The use of PET MPI in patients with high-risk coronary anomalies was rated as may be appropriate, especially if it can be performed with exercise stress testing (scenario 43) (77-79).

\section{Summary of Recommendations}

The evidence to support the use of stress-rest PET MPI in asymptomatic patients is commonly limited to indications such as an intermediate-to-high probability of the presence of CAD, an uninterpretable resting ECG, coronary artery calcifications of $\geq 400 \mathrm{HU}$, or the presence of familial hyperlipidemia in the appropriate clinical setting. Further, in asymptomatic patients with equivocal or abnormal prior test results, further testing with PET MPI is commonly needed for risk stratification and to further triage the patient's care. In asymptomatic patients with prior revascularization procedures, the recommendation is to follow the SPECT guidelines with potential application of PET MPI with a history of PCI of $>2 \mathrm{y}$ and CABG of $>5 \mathrm{y}$. Other important indications for PET MPI are seen in asymptomatic patients with CAV who are being considered for solid organ transplantation, who have cancer and LV dysfunction during or after chemotherapy or radiation therapy, who have a history of coronary vasculitis and evidence of structurally abnormal coronary arteries (e.g., aneurysms), or who have a history of high-risk coronary anomalies.

\section{SECTION 3: DIAGNOSED HEART FAILURE (RESTING LV FUNCTION PREVIOUSLY ASSESSED BUT NO PRIOR CAD EVALUATION)}

\section{Introduction}

Heart failure is a common and highly morbid condition affecting $20 \%$ of Americans over their lifetimes (71). CAD is among the most common causes of heart failure. Consequently, evaluation for CAD is an important part of the workup of most patients who are diagnosed with heart failure. Because surgical revascularization of patients with ischemic heart failure with reduced ejection fraction is associated with improved survival (80), it is critical to identify this modifiable cause of heart failure. Depending on clinical risk factors and symptoms, suspicion of CAD may be sufficiently high that traditional noninvasive testing modalities, such as SPECT MPI, may have insufficient negative predictive value to avoid further testing in many patients. Conversely, because of the greater sensitivity and specificity of PET MPI, a PET-based testing strategy may be sufficient to avoid invasive angiography. Selection of appropriate patients for such a strategy requires assessment of the clinical risk of CAD on the basis of symptoms, history, and risk factors, as well as consideration of whether the patient has reduced or preserved ejection fraction. Furthermore, although conflicting clinical data have been reported for other viability testing modalities (81), most evidence supports the use of PET MPI combined with ${ }^{18}$ F-FDG PET viability assessment for selecting patients for whom the benefits of revascularization outweigh the risks $(82,83)$.

\section{Clinical Scenarios and AUC Scores}

Table 3 presents the following clinical scenarios and final AUC scores for the use of stress and rest perfusion PET in patients with diagnosed heart failure (resting LV function previously assessed but no prior CAD evaluation).

Clinical Scenarios 44-46: Patients with Diagnosed Heart Failure with Reduced Ejection Fraction.

Scenario 44: Patients with diagnosed heart failure with reduced ejection fraction, no established history of CAD, and a low clinical risk of CAD: stress and rest perfusion PET (Score 7 - Appropriate)

Scenario 45: Patients with diagnosed heart failure with reduced ejection fraction, no established history of CAD, and an intermediate clinical risk of CAD: stress and rest perfusion PET (Score 9 - Appropriate)

Scenario 46: Patients with diagnosed heart failure with reduced ejection fraction, no established history of CAD, and a high clinical risk of CAD: stress and rest perfusion PET (Score 8 - Appropriate)

Ischemic heart disease is an important cause of heart failure with reduced ejection fraction. Patients without established CAD may, in many cases, warrant workup for ischemic causes, although this will vary depending on the clinical risk of CAD, which includes the presence of angina (71). In some cases, stress testing has been felt to be insufficiently sensitive to rule out ischemic causes for heart failure in patients with a higher clinical risk (i.e., insufficient negative predictive value). However, the greater sensitivity of stress perfusion PET, which is always combined with some form of attenuation correction, leading to a higher specificity, led the panel to rate this test as appropriate across the entire 
TABLE 3

Clinical Scenarios for the Use of Rest or Stress Perfusion PET in Patients with Diagnosed Heart Failure (Resting LV Function Previously Assessed but No Prior CAD Evaluation)

\begin{tabular}{|c|c|c|c|}
\hline Scenario no. & Description & Appropriateness & Score \\
\hline \multicolumn{4}{|c|}{ Patients with diagnosed heart failure with reduced ejection fraction } \\
\hline 44 & $\begin{array}{l}\text { Patients with diagnosed heart failure with reduced ejection fraction, no established } \\
\text { history of } C A D \text {, and a low clinical risk of } C A D\end{array}$ & Appropriate & 7 \\
\hline 45 & $\begin{array}{l}\text { Patients with diagnosed heart failure with reduced ejection fraction, no established } \\
\text { history of } C A D \text {, and an intermediate clinical risk of CAD }\end{array}$ & Appropriate & 9 \\
\hline 46 & $\begin{array}{l}\text { Patients with diagnosed heart failure with reduced ejection fraction, no established } \\
\text { history of } C A D \text {, and a high clinical risk of CAD }\end{array}$ & Appropriate & 8 \\
\hline \multicolumn{4}{|c|}{ Patients with diagnosed HFpEF } \\
\hline 47 & $\begin{array}{l}\text { Patients with diagnosed HFpEF, no established history of CAD, and a low clinical } \\
\text { risk of CAD }\end{array}$ & May be Appropriate & 5 \\
\hline 48 & $\begin{array}{l}\text { Patients with diagnosed HFpEF, no established history of } C A D \text {, and an intermediate } \\
\text { clinical risk of CAD }\end{array}$ & Appropriate & 9 \\
\hline 49 & $\begin{array}{l}\text { Patients with diagnosed HFpEF, no established history of CAD, and a high clinical } \\
\text { risk of CAD }\end{array}$ & Appropriate & 9 \\
\hline \multicolumn{4}{|c|}{ Patients with diagnosed heart failure undergoing assessment of viability and hibernation } \\
\hline 50 & $\begin{array}{l}\text { Patients with diagnosed heart failure who are undergoing assessment of viability } \\
\text { and hibernation with }{ }^{18} \mathrm{~F}-\mathrm{FDG} \text { PET: rest perfusion PET }\end{array}$ & Appropriate & 9 \\
\hline 51 & $\begin{array}{l}\text { Patients with diagnosed heart failure who are undergoing assessment of viability } \\
\text { and hibernation: rest and stress perfusion PET }\end{array}$ & Appropriate & 9 \\
\hline
\end{tabular}

spectrum of risk. Notably, the addition of quantification of MBF and flow reserve will further improve the sensitivity and negative predictive value.

Clinical Scenarios 47-49: Patients with Newly Diagnosed Heart Failure with Preserved Ejection Fraction (HFpEF).

Scenario 47: Patients with diagnosed HFpEF, no established history of CAD, and a low clinical risk of CAD: stress and rest perfusion PET (Score 5 - May be Appropriate)

Scenario 48: Patients with diagnosed HFpEF, no established history of CAD, and an intermediate clinical risk of CAD: stress and rest perfusion PET (Score 9 - Appropriate)

Scenario 49: Patients with diagnosed HFpEF, no established history of CAD, and a high clinical risk of CAD: stress and rest perfusion PET (Score 9 - Appropriate)

HFpEF is a complex syndrome with contributions from myocardial ischemia, LV hypertrophy and stiffening, vascular stiffening, renal dysfunction, and altered pulmonary and skeletal muscle function, among other systemic alterations. Several previous studies have demonstrated a link between abnormalities in microvascular function and myocardial strain, as well as with clinical HFpEF $(84,85)$. In patients with low clinical risk of $\mathrm{CAD}$, the yield of stress PET will generally be suboptimal, although in select patients, testing may be helpful. Conversely, in patients with intermediate to high clinical risk, evaluation for myocardial ischemia as a cause of or contributor to heart failure symptoms is appropriate.

Clinical Scenarios 50 and 51: Patients with Diagnosed Heart Failure Who Are Undergoing Assessment of Viability and Hibernation.

Scenario 50: Patients with diagnosed heart failure who are undergoing assessment of viability and hibernation with ${ }^{18} \mathrm{~F}$ FDG PET: rest perfusion PET (Score 9 - Appropriate)
Scenario 51: Patients with diagnosed heart failure who are undergoing assessment of viability and hibernation: rest or stress perfusion PET (Score 9 - Appropriate)

A detailed discussion of the role of rest and stress perfusion PET in the assessment of viability and hibernation can be found in scenario 164.

\section{Summary of Recommendations}

Rest-stress PET MPI is an appropriate test for the evaluation of CAD in many patients with heart failure, although careful consideration of clinical risk and ejection faction are warranted to optimize testing efficiency and minimize complications.

\section{SECTION 4: EVALUATION OF PATIENTS WITH KNOWN OR SUSPECTED CARDIAC SARCOIDOSIS}

\section{Introduction}

This section addresses the value of rest and stress PET MPI, along with ${ }^{18}$ F-FDG PET imaging, for the evaluation of patients with known or suspected cardiac sarcoidosis.

\section{Background}

${ }^{18}$ F-FDG PET imaging has emerged as a powerful tool for the evaluation of myocardial inflammation in patients with known or suspected cardiac sarcoidosis. MPI is an integral part of the study protocol for ${ }^{18}$ F-FDG PET imaging for cardiac sarcoidosis. An abnormal resting myocardial perfusion pattern in a typical or atypical location, in conjunction with ${ }^{18} \mathrm{~F}$-FDG PET, increases diagnostic accuracy and enhances the accuracy of clinical outcome prediction in these patients. Adding stress to rest perfusion imaging may be indicated to identify flow-limiting CAD lesions in symptomatic patients with known or suspected CAD, as outlined in section 1. Although 2 recent multisocietal consensus documents $(86,87)$ discussed the 
clinical indications and procedural aspects of ${ }^{18} \mathrm{~F}-\mathrm{FDG}$ PET for cardiac sarcoidosis, the appropriate use of MPI in the context of ${ }^{18} \mathrm{~F}-\mathrm{FDG}$ PET for cardiac sarcoidosis has not been previously described.

\section{Clinical Scenarios and AUC Scores}

Table 4 presents the following clinical scenarios and final AUC scores for the use of rest or stress perfusion PET in the evaluation of patients with known or suspected cardiac sarcoidosis.

Clinical Scenarios 52-55: Evaluation of Patients with Known or Suspected Cardiac Sarcoidosis.

Scenario 52: Patients undergoing assessment of myocardial inflammation with ${ }^{18}$ F-FDG PET: rest perfusion PET (Score 9 - Appropriate)

Scenario 53: Patients with suspected cardiac sarcoidosis who have not been previously evaluated for CAD, in whom myocardial inflammation imaging with ${ }^{18} \mathrm{~F}$-FDG PET is planned: stress and rest perfusion PET (Score 5 - May be Appropriate)

Scenario 54: Patients with suspected cardiac sarcoidosis who have been previously evaluated for CAD in whom myocardial inflammation imaging with ${ }^{18} \mathrm{~F}$-FDG PET is planned: stress and rest perfusion PET (Score 1 - Rarely Appropriate)

Scenario 55: Patients undergoing reevaluation for response to therapy or recurrent inflammation with ${ }^{18} \mathrm{~F}-\mathrm{FDG}$ PET: rest perfusion PET (Score 9 - Appropriate)

Rest MPI along with ${ }^{18} \mathrm{~F}$-FDG PET imaging is essential for the evaluation of cardiac sarcoidosis. A pattern of perfusion metabolic mismatch may be seen in active myocardial sarcoidosis, as well as in hibernating myocardium. Hence, knowledge of the presence or absence of coronary artery obstruction is essential to the appropriate interpretation of the perfusion and metabolic patterns in patients with suspected sarcoidosis. For diagnosing CAD, imaging of the coronary arteries with either invasive or CT-based imaging is preferred over stress perfusion imaging; stress-rest MPI is an alternative to angiography. Some experts have expressed concern over same-day ${ }^{18} \mathrm{~F}$-FDG and stress testing, as ischemia may promote glycolysis in noninflamed myocardium. Others, however, felt that this effect could be surmounted by use of vasodilator stress, careful comparison of inducible perfusion defects with ${ }^{18}$ F-FDG uptake, or temporal separation of stress testing and ${ }^{18} \mathrm{~F}$-FDG injection.
Because of the variable success rates in the suppression of physiologic ${ }^{18}$ F-FDG uptake by normal myocardium, rest perfusion imaging is helpful and may improve specificity, especially during reevaluation for response to therapy. When myocardial ${ }^{18} \mathrm{~F}$ FDG uptake is present on the follow-up scan (persistent inflammation), a decrease in perfusion defect size may indicate partial response. When myocardial ${ }^{18} \mathrm{~F}$-FDG uptake is absent on the follow-up scan, the presence of a perfusion defect may indicate fibrosis (worsening), whereas the absence of a perfusion defect may indicate improvement with resolution of myocardial inflammation.

Detailed discussion of the role of rest and stress perfusion PET in the assessment of cardiac sarcoidosis can be found in scenarios 162 and 163.

\section{Summary of Recommendations}

Rest PET MPI was rated by the experts as appropriate in patients undergoing assessment of myocardial inflammation with ${ }^{18}$ F-FDG PET at baseline and during reevaluation for response to therapy or recurrent inflammation. In contrast, stress MPI was rated as may be appropriate in the evaluation of patients with suspected sarcoidosis who have not been previously evaluated for CAD, and as rarely appropriate in patients with suspected sarcoidosis who have been previously evaluated for CAD.

\section{SECTION 5: EVALUATION OF ARRHYTHMIAS WITHOUT ISCHEMIC EQUIVALENT (NO PRIOR CARDIAC EVALUATION)}

\section{Introduction}

Ischemia is a critical causal factor in a relatively small subset of arrhythmias. Most common arrhythmias such as AF are not believed to primarily be a consequence of myocardial ischemia. Nonetheless, evaluation for ischemia is often clinically necessary for patients with AF accompanied by ischemic symptoms, in patients treated with antiarrhythmic drugs, and in patients being considered for some invasive therapies. Furthermore, ischemia is a primary cause of ventricular fibrillation (VF), and prior infarctions may provide a substrate for ventricular tachycardia (VT) and premature ventricular contractions (PVCs). Consequently, many patients with arrhythmia may be referred for evaluation of CAD either invasively or noninvasively. Some of these patients may be appropriately evaluated with PET MPI, depending on clinical stability, clinical risk of CAD, and type of arrhythmia.

TABLE 4

Clinical Scenarios for the Use of Rest or Stress Perfusion PET in the Evaluation of Patients with Known or Suspected Cardiac Sarcoidosis

\begin{tabular}{|c|c|c|c|}
\hline Scenario no. & Description & Appropriateness & Score \\
\hline 52 & $\begin{array}{l}\text { Patients undergoing assessment of myocardial inflammation with }{ }^{18} \mathrm{~F}-\mathrm{FDG} \mathrm{PET} \text { : } \\
\text { rest perfusion PET }\end{array}$ & Appropriate & 9 \\
\hline 53 & $\begin{array}{l}\text { Evaluation of patients with suspected cardiac sarcoidosis who have not been } \\
\text { previously evaluated for CAD in whom myocardial inflammation imaging with } \\
{ }^{18} \mathrm{~F}-\mathrm{FDG} \text { PET is planned: stress and rest perfusion PET }\end{array}$ & May be Appropriate & 5 \\
\hline 54 & $\begin{array}{l}\text { Evaluation of patients with suspected cardiac sarcoidosis who have been } \\
\text { previously evaluated for CAD in whom myocardial inflammation imaging } \\
\text { with }{ }^{18} \mathrm{~F}-\mathrm{FDG} \text { PET is } \\
\text { planned: stress and rest perfusion PET }\end{array}$ & Rarely Appropriate & 1 \\
\hline 55 & $\begin{array}{l}\text { Patients undergoing reevaluation for response to therapy or recurrent } \\
\text { inflammation with }{ }^{18} \mathrm{~F} \text {-FDG PET: rest perfusion PET }\end{array}$ & Appropriate & 9 \\
\hline
\end{tabular}


TABLE 5

Clinical Scenarios for the Use of Stress Perfusion PET in the Evaluation of Arrhythmias Without Ischemic Equivalent (No Prior Cardiac Evaluation)

\begin{tabular}{|c|c|c|c|}
\hline Scenario no. & Description & Appropriateness & Score \\
\hline \multicolumn{4}{|c|}{ Clinically stable patients with sustained VT } \\
\hline 56 & $\begin{array}{l}\text { Clinically stable patients with an episode of sustained VT who have a low } \\
\text { global clinical risk of CAD }\end{array}$ & May be Appropriate & 6 \\
\hline 57 & $\begin{array}{l}\text { Clinically stable patients with an episode of sustained VT who have an } \\
\text { intermediate clinical risk of CAD }\end{array}$ & Appropriate & 9 \\
\hline 58 & $\begin{array}{l}\text { Clinically stable patients with an episode of sustained VT who have a high } \\
\text { clinical risk of CAD }\end{array}$ & Appropriate & 8 \\
\hline 59 & Clinically unstable patients with an episode of sustained VT & Rarely Appropriate & 1 \\
\hline \multicolumn{4}{|c|}{ Patients with a recent episode of VF } \\
\hline 60 & $\begin{array}{l}\text { Clinically stable patients with a recent episode of VF who have a low clinical } \\
\text { risk of CAD }\end{array}$ & Rarely Appropriate & 1 \\
\hline 61 & Clinically unstable patients with a recent episode of VF & Rarely Appropriate & 1 \\
\hline \multicolumn{4}{|c|}{ Patients with exercise-induced VT or nonsustained VT } \\
\hline 62 & Patients with nonsustained VT who have a low clinical risk of CAD & May be Appropriate & 4 \\
\hline 63 & Patients with exercise-induced VT who have a low clinical risk of CAD & May be Appropriate & 5 \\
\hline 64 & Patients with nonsustained VT who have an intermediate clinical risk of CAD & Appropriate & 7 \\
\hline 65 & Patients with exercise-induced VT who have an intermediate clinical risk of CAD & Appropriate & 8 \\
\hline 66 & Patients with nonsustained VT who have a high clinical risk of CAD & Appropriate & 8 \\
\hline 67 & Patients with exercise-induced VT who have a high clinical risk of CAD & May be Appropriate & 4 \\
\hline \multicolumn{4}{|c|}{ Patients with frequent PVCs } \\
\hline 68 & Patients with frequent PVCs who have a low clinical risk of CAD & Appropriate & 7 \\
\hline 69 & Patients with frequent PVCs who have an intermediate clinical risk of CAD & Appropriate & 8 \\
\hline 70 & Patients with frequent PVCs who have a high clinical risk of CAD & Rarely Appropriate & 2 \\
\hline \multicolumn{4}{|c|}{ Patients with infrequent PVCs } \\
\hline 71 & Patients with infrequent PVCs who have a low clinical risk of CAD & Rarely Appropriate & 1 \\
\hline 72 & Patients with infrequent PVCs who have an intermediate clinical risk of CAD & May be Appropriate & 5 \\
\hline 73 & Patients with infrequent PVCs who have a high clinical risk of CAD & May be Appropriate & 5 \\
\hline \multicolumn{4}{|c|}{ Patients with new-onset AF } \\
\hline 74 & Patients with new-onset $A F$ who have a low global clinical risk of $C A D$ & Rarely Appropriate & 2 \\
\hline 75 & Patients with new-onset $A F$ who have an intermediate global clinical risk of $C A D$ & May be Appropriate & 5 \\
\hline 76 & Patients with new-onset $A F$ who have a high global clinical risk of CAD & May be Appropriate & 6 \\
\hline \multicolumn{4}{|c|}{ Evaluation of patients before the initiation of antiarrhythmic medications } \\
\hline 77 & Patients with a low global CAD risk before initiation of antiarrhythmic medications & May be Appropriate & 5 \\
\hline 78 & $\begin{array}{l}\text { Patients with an intermediate global CAD risk before initiation of antiarrhythmic } \\
\text { medications }\end{array}$ & May be Appropriate & 6 \\
\hline 79 & Patients with a high global CAD risk before initiation of antiarrhythmic medications & Appropriate & 7 \\
\hline
\end{tabular}

\section{Clinical Scenarios and AUC Scores}

Table 5 presents the following clinical scenarios and final AUC scores for the use of stress perfusion PET in the evaluation of patients with arrhythmias without an ischemic equivalent (no prior cardiac evaluation).

Clinical Scenarios 56-59: Clinically Stable Patients with Sustained VT.

Scenario 56: Clinically stable patients with an episode of sustained VT who have a low global clinical risk of CAD (Score 6 - May be Appropriate)

Scenario 57: Clinically stable patients with an episode of sustained VT who have an intermediate clinical risk of CAD (Score 9 - Appropriate)
Scenario 58: Clinically stable patients with an episode of sustained VT who have a high clinical risk of CAD (Score 8 Appropriate)

Scenario 59: Clinically unstable patients with an episode of sustained VT (Score 1 - Rarely Appropriate)

Transient myocardial ischemia is more commonly a trigger of polymorphic rather than monomorphic sustained VT. Monomorphic VT in the setting of prior myocardial infarction (MI) is typically due to scar-related reentry and not to acute ischemia. For patients suspected of having myocardial ischemia, stress testing or coronary angiography along with subsequent revascularization should be performed before catheter ablation when possible to 
avoid significant ischemia during VT induction, mapping, and ablation. In clinically stable patients with an episode of sustained VT, the use of stress perfusion PET is thought to be dependent on the global clinical risk of CAD in the patient. Patients with a low global clinical risk are given an AUC score of 6, may be appropriate. However, in patients considered to be at intermediate or high clinical risk, stress perfusion PET is considered appropriate. It is important to realize that in clinically unstable patients, stress perfusion PET may occasionally contribute to assessment but is more likely to delay definitive clinical care. Consequently, it was considered rarely appropriate, irrespective of the clinical risk of CAD.

Clinical Scenarios 60 and 61: Patients with a Recent Episode of VF.

Scenario 60: Clinically stable patients with a recent episode of VF who have a low clinical risk of CAD (Score 1 - Rarely Appropriate)

Scenario 61: Clinically unstable patients with a recent episode of VF (Score 1 - Rarely Appropriate)

In clinically stable and unstable patients with a recent episode of VF who have a low clinical risk of CAD, stress perfusion PET is thought to be rarely appropriate, as it may result in delay of definitive therapy and will only rarely change the clinical management.

Clinical Scenarios 62-67: Patients with Exercise-Induced VT or Nonsustained VT.

Scenario 62: Patients with nonsustained VT who have a low clinical risk of CAD (Score 4 - May be Appropriate)

Scenario 63: Patients with exercise-induced VT who have a low clinical risk of CAD (Score 5 - May be Appropriate)

Scenario 64: Patients with nonsustained VT who have an intermediate clinical risk of CAD (Score 7 - Appropriate)

Scenario 65: Patients with exercise-induced VT who have an intermediate clinical risk of CAD (Score 8 - Appropriate)

Scenario 66: Patients with nonsustained VT who have a high clinical risk of CAD (Score 8 - Appropriate)

Scenario 67: Patients with exercise-induced VT who have a high clinical risk of CAD (Score 4 - May be Appropriate)

Exercise-induced VT rarely occurs in the absence of structural heart disease. In its absence, idiopathic monomorphic VT has a relatively benign prognosis. The main aspect of the risk stratification process is recognizing subtle forms of organic heart disease such as arrhythmogenic right ventricular cardiomyopathy. Exercise-induced polymorphic VT is often malignant and may be related to myocardial ischemia. Exercise-induced polymorphic VT has also been seen in mitral valve prolapse (88). Patients with stable coronary disease may have short bursts of polymorphic VT during exercise tests that are not reproducible during repeated testing and that have unknown long-term clinical significance. Therefore, the appropriateness of stress perfusion PET increases with increasing risk of clinical CAD. However, among those with high clinical risk and exercise-induced VT, appropriateness is somewhat lower, as noninvasive testing may result in delay of definitive therapy.

Clinical Scenarios 68-70: Patients with Frequent PVCs.

Scenario 68: Patients with frequent PVCs who have a low clinical risk of CAD (Score 7 - Appropriate)
Scenario 69: Patients with frequent PVCs who have an intermediate clinical risk of CAD (Score 8 - Appropriate)

Scenario 70: Patients with frequent PVCs who have a high clinical risk of CAD (Score 2 - Rarely Appropriate)

PVCs are the most common ventricular arrhythmia. Their prognostic significance cannot be interpreted without considering the presence or absence of any associated underlying cardiac condition. In the absence of structural heart disease, PVCs were generally considered to be benign. In the 1970s and 1980s, it was postulated that frequent PVCs could be a trigger for VT, VF, and sudden cardiac death after an MI, and therefore PVC suppression was thought to be warranted in this context. In a normal healthy population, PVCs have been observed in up to $75 \%$ of subjects during 48-h Holter monitoring (89), with $>60 \mathrm{PVCs} / \mathrm{h}$ detected in up to $4 \%$ of individuals $(90)$. This latter prevalence increases progressively with age, comorbidity burden, and duration of monitoring, ranging from $1 \%$ to $69 \%(91,92)$. The adverse impact of frequent PVCs on the prognosis in patients with underlying or structural cardiac disease, such as a previous MI, is well established (93). In the late 1990s, Duffee et al. demonstrated that pharmacologic suppression of PVCs in patients with presumed idiopathic dilated cardiomyopathy subsequently improved LVEF (94). Recent studies have demonstrated the potential detrimental effects of frequent PVCs in patients with structurally normal hearts and the development and reversibility of PVC-induced cardiomyopathy $(95,96)$. Frequent PVCs can also worsen a preexisting cardiomyopathy, in which case PVC suppression may only lead to partial recovery of LV dysfunction (97). A PVC burden $>24 \%$ has been suggested to have the highest sensitivity and specificity (79\% and $78 \%$, respectively) in predicting the occurrence of PVC-induced cardiomyopathy (96). However, a recent study has shown that heart failure may be caused by a much lower PVC burden than that traditionally associated with PVC-induced cardiomyopathy (98). In the presence of LVEF impairment or regional wall motion abnormalities, stress imaging may be performed in patients with impaired LV systolic function, depending on their cardiovascular risk profile. Therefore, in patients with frequent PVCs with low or intermediate clinical risk of $\mathrm{CAD}$, stress perfusion is appropriate. Stress perfusion in patients with frequent PVCs and a high clinical risk of CAD is rarely appropriate, as it may delay definitive therapy.

Clinical Scenarios 71-73: Patients with Infrequent PVCs.

Scenario 71: Patients with infrequent PVCs who have a low clinical risk of CAD (Score 1 - Rarely Appropriate)

Scenario 72: Patients with infrequent PVCs who have an intermediate clinical risk of CAD (Score 5 - May be Appropriate)

Scenario 73: Patients with infrequent PVCs who have a high clinical risk of CAD (Score 5 - May be Appropriate)

As stated earlier, the prognostic significance of PVCs cannot be interpreted without considering the presence or absence of any associated underlying cardiac condition. However, a low PVC burden has no prognostic significance. Therefore, patients with infrequent PVCs are to be evaluated according their clinical risk of CAD; that is, stress PET perfusion in patients with infrequent PVCs and a low clinical risk of CAD is rarely appropriate, and stress PET perfusion in patients with an intermediate to high clinical risk may be appropriate in certain circumstances, depending on associated clinical factors. 
Clinical Scenarios 74-76: Patients with New-Onset AF.

Scenario 74: Patients with new-onset AF who have a low global clinical risk of CAD (Score 2 - Rarely Appropriate)

Scenario 75: Patients with new-onset AF who have an intermediate global clinical risk of CAD (Score 5 - May be Appropriate)

Scenario 76: Patients with new-onset AF who have a high global clinical risk of CAD (Score 6 - May be Appropriate)

$\mathrm{CAD}$ is only one of many risk factors associated with the development of AF. Patients with recent-onset AF should be evaluated according to their clinical risk of $\mathrm{CAD}$ and in relation to their LVEF. Therefore, in patients with new-onset AF who have a low global clinical risk of CAD, stress perfusion PET is rarely appropriate. However, in patients with new-onset AF who have an intermediate to high global clinical risk of $\mathrm{CAD}$, stress perfusion PET may be appropriate, depending on the presence of LV systolic or diastolic dysfunction (99).

Clinical Scenarios 77-79: Evaluation of Patients Before the Initiation of Antiarrhythmic Medications.

Scenario 77: Patients with a low global CAD risk before initiation of antiarrhythmic medications (Score 5 - May be Appropriate)

Scenario 78: Patients with an intermediate global CAD risk before initiation of antiarrhythmic medications (Score 5 - May be Appropriate)

Scenario 79: Patients with a high global CAD risk before initiation of antiarrhythmic medications (Score 7 - Appropriate)

The safety of antiarrhythmic drug therapy typically determines the initial choice of antiarrhythmic drugs. The 2016 ESC Guidelines for the Management of Atrial Fibrillation (99) emphasize the assessment of risks of harm from ventricular arrhythmogenesis before initiation of long-term rhythm control therapy, the goal being to improve symptoms in AF safely. Appropriate options are related to specific patient characteristics, including the global clinical risk of CAD among others, with appropriateness of stress perfusion PET increasing with increasing clinical CAD risk (99).

\section{Summary of Recommendations}

Appropriateness of PET MPI for the evaluation of CAD in patients with arrhythmias varies greatly, depending on the clinical risk of CAD, patient stability, and type of arrhythmia. Consequently, careful consideration is required for optimal patient selection.

\section{SECTION 6: SYNCOPE WITHOUT ISCHEMIC EQUIVALENT}

\section{Introduction}

In patients with syncope, it is important to differentiate cardiovascular causes (i.e., bradycardia, tachycardia, hypotension due to poor cardiac output, obstructions to blood flow, or arterial dissection) from noncardiac causes (e.g., volume depletion, blood loss, neurally mediated syncope) (100). Syncope that occurs in the setting of heart disease or during exertion is more likely to be of cardiovascular etiology. It is important to understand that many cardiovascular causes of syncope are serious and carry a high risk of life-threatening or life-altering complications. MI and ischemia are uncommon causes of syncope, especially outside of aortic dissection. Consequently, the appropriateness of stress perfusion PET varies with the patient's global clinical risk of CAD and other related clinical factors, such as whether the syncope occurred during exertion.

\section{Clinical Scenarios and AUC Scores}

Table 6 presents the following clinical scenarios and final AUC scores for the use of stress perfusion PET in patients with syncope without an ischemic equivalent.

Clinical Scenarios 80-82: Patients With Syncope Without an Ischemic Equivalent.

Scenario 80: Patients with syncope and a low global clinical risk of CAD (Score 2 - Rarely Appropriate)

Scenario 81: Patients with syncope and an intermediate global clinical risk of CAD (Score 5 - May be Appropriate)

Scenario 82: Patients with syncope and a high global clinical risk of CAD (Score 7 - Appropriate)

\section{Summary of Recommendations}

Outside of a massive MI, CAD is a rare cause of syncope and consequently the diagnostic focus should be elsewhere in patients with a low clinical risk of CAD. However, in subsets of patients with intermediate and higher clinical risk, PET MPI evaluation for ischemic contributions to patients with ischemia may be appropriate.

\section{SECTION 7: ASSESSMENT OF CMD IN SYMPTOMATIC PATIENTS}

\section{Introduction}

This section evaluates the role of PET MPI in the assessment of CMD in patients with angina or chest pain symptoms who have obstructive or nonobstructive hypertrophic cardiomyopathy (HCM), known LV hypertrophy, diabetes mellitus, obesity, or syndrome X. The role of PET MPI is also evaluated in the assessment of CMD in postmenopausal women with these symptoms.

ICA and noninvasive coronary angiography are commonly performed for the evaluation of obstructive CAD in patients with angina pectoris (chest tightness) or shortness of breath. In the United States, approximately 4 million invasive coronary angiograms are performed each year for diagnostic purposes (101). In these symptomatic patients undergoing invasive diagnostic coronary angiography, up to $60 \%$ may have no CAD or nonobstructive epicardial CAD (defined as a lesion with a stenosis diameter of $<50 \%$ ) $(101,102)$. A substantial portion of these symptomatic patients with nonobstructive $\mathrm{CAD}$ may have underlying $\mathrm{CMD}$ as the functional substrate of their symptoms (102-107). This large subgroup of patients with chest pain symptoms and CMD (microvascular angina or vasospastic angina) commonly presents a substantial increase in morbidity $(13,101,108)$ and impaired quality of life $(109)$ and thus poses an important health-care concern (110). Invasive testing of CMD, with intracoronary acetylcholine application, for example, is time-consuming and not risk free (111). For this reason, such an invasive approach is rarely applied in a busy catheterization laboratory and commonly reserved for research purposes. Cardiac PET with various positron-emitting flow radiotracers $\left({ }^{82} \mathrm{Rb}\right.$ or ${ }^{13} \mathrm{~N}$-ammonia) affords the unique advantage of concurrently assessing myocardial perfusion and MBF in milliliters per gram per minute (2). Assessing hyperemic MBF during pharmacologic vasodilation and at rest enables the calculation of the MFR (MFR = MBF hyperemia/MBF rest) and thus the noninvasive assessment of microvascular function. Notably, normal stress perfusion (or absence of 


\begin{tabular}{llll}
\hline Scenario no. & & Description & Appropriateness \\
\hline 80 & Patients with syncope and a low global clinical risk of CAD & Rarely Appropriate \\
81 & Patients with syncope and an intermediate global clinical risk of CAD & May be Appropriate & 2 \\
82 & Patients with syncope and a high global clinical risk of CAD & Appropriate & 7 \\
\hline
\end{tabular}

regional ischemia), as determined with PET, widely signifies the absence of obstructive epicardial CAD, and the concurrent determination of hyperemic MBF and MFR affords the evaluation of the CMD. Consequently, cardiac PET perfusion and flow assessment may be applied not only to patients without evidence of obstructive CAD on coronary angiography, but also directly to patients with a likelihood of CMD without having to undergo coronary angiography. In this respect, it is important to note that the identification and characterization of microvascular disease by PET flow studies has been recognized to carry important diagnostic and prognostic information that likely affects the treatment decision process and treatment options $(2,13,15,39,46,112,113)$.

\section{Background}

Given the wide variety of clinical manifestations in patients with microvascular angina pectoris, the Coronary Vasomotion Disorders International Study Group (COVADIS) recently established standardized criteria for microvascular angina pectoris related to $\mathrm{CMD}$ (102). According to COVADIS, the criteria for diagnosing microvascular angina pectoris requires the presence of (1) symptoms of myocardial ischemia, (2) absence of obstructive CAD, (3) objective evidence of myocardial ischemia, and (4) evidence of CMD. Proof of impaired hyperemic MBF or MFR, indicative of CMD, has therefore become an integral part of the diagnosis of microvascular angina pectoris in patients with chest pain syndrome. An important consideration is that CMD can be present either as a consequence of detrimental effects of classic cardiovascular risk factors, such as hypercholesterolemia, arterial hypertension, diabetes mellitus, or smoking, or as a result of myocardial disease, such as occurs in obstructive and nonobstructive HCM or secondary cardiomyopathy because of valvular dysfunction with hemodynamic alterations. In particular, LV hypertrophy or structural alterations, such as increased interstitial and perivascular fibrosis, decreased capillary density, and increased arterial stiffness, may be induced by arterial hypertension, diabetes mellitus, or obesity, potentially leading to CMD. Overall, PET assessment of CMD in these symptomatic patients has been demonstrated to convey important diagnostic and prognostic information about future outcomes $(3,16,29,39,46,48,70,102,104,105,114-120)$, which emphasizes the central role of a disturbance in microvascular function in affecting the cardiovascular outcome. As CMD is amenable to various treatment options $(2,3,121)$, its noninvasive detection in symptomatic patients seems to be pivotal in the treatment decision process, which not only may improve symptoms, but likely improves cardiovascular outcome as well, which warrants further clinical evaluation. A variety of medical treatment options are available to address symptomatic CMD $(2,3,114,121)$. In patients with traditional cardiovascular risk factors, starting treatment or enhancing its intensity or dose by using, for example, angiotensin-converting enzyme inhibitors (ACE-Is) or statins, or achieving tight glucose control in diabetic patients, may be the best first step in improving CMD and thereby flow, likely also improving symptoms (13). If these attempts are without success, ranolazine or calcium-channel blockers may offer additional treatment options. For more detailed information and recommendations, see reference 121 .

\section{Clinical Scenarios and AUC Scores}

Table 7 presents the following clinical scenarios and final AUC scores for the use of PET MPI in the assessment of CMD in symptomatic patients.

Clinical Scenarios 83-87: Symptomatic Patients with Known Obstructive or Nonobstructive HCM. In patients with obstructive or nonobstructive HCM (scenarios 83-87), CMD is frequently encountered that accounts for angina or chest pain symptoms and that also conveys important prognostic information $(13,102,104,105,115-117,119)$. PET-determined CMD is helpful here to identify those HCM patients who are likely to benefit, at least in part, from intensified medical treatment with vasoactive medications such as ACE-I, angiotensin type 2 receptor blocker (ARB), calcium-channel blockers, or ranolazine in order to reduce symptoms and improve outcome; this approach does, however, still require large-scale evaluation $(3,121-124)$. Commonly, classic CAD is less likely to be present in this relatively young population with a lower burden of cardiovascular risk factors. Nonetheless, obstructive CAD lesions may be present in up to $26 \%$ of these patients, which may manifest as regional perfusion deficit on PET MPI images and require further evaluation with either noninvasive coronary angiography or ICA $(125,126)$. Overall, from the available prevalence and outcome data for CMD in patients with HCM, and, in particular, from standard clinical practice regarding the clinical decision-making process and treatment options for controlling symptoms, these scenarios were commonly deemed appropriate.

The following scenarios address symptomatic patients with positive or negative results of an exercise ECG stress test.

Scenario 83: Symptomatic patients with positive results of an exercise ECG stress test (Score 8 - Appropriate)

Symptomatic patients with HCM and CMD have an increased risk for cardiac arrhythmia and for a worse outcome. Positive results of an ECG stress test are nonspecific because stress-related disturbances can be related not only to ischemia, but also to wall thickening or interstitial fibrosis. In these symptomatic patients with HCM, stress-rest perfusion PET can be performed to assess the presence of flow-limiting obstructive CAD and, at the same time, to identify CMD as a potential substrate for angina symptoms $(102,105)$. Such symptomatic patients without obstructive CAD but with CMD may benefit from ACE-I, ARB, or ranolazine to improve hyperemic flow and symptoms $(2,85,121-124,126,127)$. From the available outcome data for CMD in this population $(115,117,119)$ and, in particular, from the point of view of the clinical decisionmaking process and treatment options, this scenario was scored as appropriate (score 8). 
TABLE 7

Clinical Scenarios for the Use of PET MPI in the Assessment of CMD in Symptomatic Patients

\begin{tabular}{|c|c|c|c|}
\hline Scenario no. & Description & Appropriateness & Score \\
\hline \multicolumn{4}{|c|}{ Symptomatic patients with known obstructive or nonobstructive HCM } \\
\hline 83 & Symptomatic patients with positive results of an exercise ECG stress test & Appropriate & 8 \\
\hline 84 & Symptomatic patients with negative results of an exercise ECG stress test & Appropriate & 8 \\
\hline 85 & $\begin{array}{l}\text { Symptomatic patients with positive or negative results of an exercise ECG } \\
\text { stress test and exclusion of obstructive CAD by angiography }\end{array}$ & Appropriate & 8 \\
\hline 86 & $\begin{array}{l}\text { Symptomatic patients with positive or negative results of an exercise ECG } \\
\text { stress test and normal SPECT perfusion findings }\end{array}$ & Appropriate & 7 \\
\hline 87 & $\begin{array}{l}\text { Asymptomatic patients with positive or negative results of an exercise ECG } \\
\text { stress test }\end{array}$ & Rarely Appropriate & 3 \\
\hline \multicolumn{4}{|c|}{ Symptomatic patients with known LV hypertrophy related to arterial hypertension, diabetes mellitus, or obesity } \\
\hline \multicolumn{4}{|c|}{ Symptomatic patients with arterial hypertension } \\
\hline 88 & Symptomatic patients with positive results of an exercise ECG stress test & Appropriate & 9 \\
\hline 89 & Symptomatic patients with negative results of an exercise ECG stress test & Appropriate & 9 \\
\hline 90 & $\begin{array}{l}\text { Symptomatic patients with positive or negative results of an exercise ECG } \\
\text { stress test and without evidence of CAD by angiography }\end{array}$ & Appropriate & 9 \\
\hline 91 & Asymptomatic patients with positive results of an exercise ECG stress test & May be Appropriate & 4 \\
\hline 92 & Asymptomatic patients with negative results of an exercise ECG stress test & Rarely Appropriate & 1 \\
\hline 93 & $\begin{array}{l}\text { Symptomatic patients with positive or negative results of an exercise ECG } \\
\text { stress test and exclusion of coronary atherosclerosis by angiography }\end{array}$ & Rarely Appropriate & 2 \\
\hline 94 & $\begin{array}{l}\text { Symptomatic patients with positive or negative results of an exercise ECG stress } \\
\text { test who have atherosclerosis and nonobstructive CAD as shown by angiography }\end{array}$ & Appropriate & 8 \\
\hline \multicolumn{4}{|c|}{ Symptomatic patients with diabetes mellitus } \\
\hline 95 & Symptomatic patients with positive results of an exercise ECG stress test & Appropriate & 8 \\
\hline 96 & Symptomatic patients with negative results of an exercise ECG stress test & Appropriate & 8 \\
\hline 97 & $\begin{array}{l}\text { Symptomatic patients with positive or negative results of an exercise ECG stress } \\
\text { test who have atherosclerosis and nonobstructive CAD as shown by angiography }\end{array}$ & Appropriate & 8 \\
\hline 98 & $\begin{array}{l}\text { Symptomatic patients with positive or negative results of an exercise ECG } \\
\text { stress test and normal SPECT perfusion findings }\end{array}$ & Appropriate & 8 \\
\hline 99 & Asymptomatic patients with positive results of an exercise ECG stress test & Appropriate & 7 \\
\hline 100 & Asymptomatic patients with negative results of an exercise ECG stress test & Rarely Appropriate & 1 \\
\hline \multicolumn{4}{|c|}{ Symptomatic patients with obesity } \\
\hline 101 & Symptomatic patients with positive results of an exercise ECG stress test & Appropriate & 9 \\
\hline 102 & Symptomatic patients with negative results of an exercise ECG stress test & Appropriate & 9 \\
\hline 103 & $\begin{array}{l}\text { Symptomatic patients with positive or negative results of an exercise ECG stress } \\
\text { test who have atherosclerosis and nonobstructive CAD as shown by angiography }\end{array}$ & Appropriate & 8 \\
\hline 104 & $\begin{array}{l}\text { Symptomatic patients with positive or negative results of an exercise ECG stress } \\
\text { test who do not have atherosclerotic CAD as shown by angiography }\end{array}$ & Rarely Appropriate & 2 \\
\hline 105 & $\begin{array}{l}\text { Symptomatic patients with positive or negative exercise ECG stress test } \\
\text { and normal SPECT perfusion findings }\end{array}$ & Appropriate & 8 \\
\hline 106 & Asymptomatic patients with positive results of an exercise ECG stress test & May be Appropriate & 5 \\
\hline 107 & Asymptomatic patients with negative results of an exercise ECG stress test & Rarely Appropriate & 1 \\
\hline \multicolumn{4}{|c|}{ Symptomatic postmenopausal women } \\
\hline 108 & $\begin{array}{l}\text { Symptomatic postmenopausal women with positive results of an exercise } \\
\text { ECG stress test }\end{array}$ & Appropriate & 9 \\
\hline 109 & $\begin{array}{l}\text { Symptomatic postmenopausal women with negative results of an exercise } \\
\text { ECG stress test }\end{array}$ & Appropriate & 9 \\
\hline 110 & $\begin{array}{l}\text { Symptomatic postmenopausal women with positive or negative results of an } \\
\text { exercise ECG stress test and exclusion of coronary atherosclerosis } \\
\text { by angiography }\end{array}$ & Rarely Appropriate & 1 \\
\hline
\end{tabular}




\begin{tabular}{|c|c|c|c|}
\hline Scenario no. & Description & Appropriateness & Score \\
\hline 111 & $\begin{array}{l}\text { Symptomatic postmenopausal women with positive or negative results of an } \\
\text { exercise ECG stress test who have atherosclerosis and nonobstructive } \\
\text { CAD as shown by angiography }\end{array}$ & Appropriate & 9 \\
\hline 112 & $\begin{array}{l}\text { Symptomatic postmenopausal women with positive or negative results of } \\
\text { an exercise ECG stress test and normal SPECT perfusion findings }\end{array}$ & Appropriate & 9 \\
\hline 113 & $\begin{array}{l}\text { Asymptomatic postmenopausal women with positive results of an exercise } \\
\text { ECG stress test }\end{array}$ & May be Appropriate & 5 \\
\hline 114 & $\begin{array}{l}\text { Asymptomatic postmenopausal women with negative results of an exercise } \\
\text { ECG stress test }\end{array}$ & Rarely Appropriate & 1 \\
\hline \multicolumn{4}{|c|}{ Symptomatic patients with syndrome $X$ : no obstructive CAD but ongoing chest pain syndrome } \\
\hline 115 & $\begin{array}{l}\text { Patients with ongoing chest pain syndrome and positive results of an exercise } \\
\text { ECG stress test who have nonobstructive CAD as shown by coronary angiography }\end{array}$ & Appropriate & 9 \\
\hline 116 & $\begin{array}{l}\text { Patients with ongoing chest pain syndrome and negative results of an exercise } \\
\text { ECG stress test who have no evidence of obstructive CAD as shown by } \\
\text { coronary angiography }\end{array}$ & Appropriate & 8 \\
\hline 117 & $\begin{array}{l}\text { Patients with ongoing chest pain syndrome, positive results of an exercise } \\
\text { ECG stress test, and normal SPECT perfusion findings }\end{array}$ & Appropriate & 9 \\
\hline 118 & $\begin{array}{l}\text { Patients with ongoing chest pain syndrome, negative results of an exercise } \\
\text { ECG stress test, and normal SPECT perfusion findings }\end{array}$ & Appropriate & 8 \\
\hline
\end{tabular}

Scenario 84: Symptomatic patients with negative results of an exercise ECG stress test (Score 8 - Appropriate)

Symptomatic patients with HCM and CMD have an increased risk for cardiac arrhythmia and for a worse outcome. Negative results of an ECG stress test may not exclude ischemia or CMD in symptomatic patients with HCM. In these patients, stress-rest perfusion PET can be performed to assess the presence of flow-limiting obstructive CAD and, at the same time, to identify CMD as a potential substrate for angina symptoms $(102,105)$. Such symptomatic patients without obstructive CAD but with CMD may benefit from ACE-I, ARB, or ranolazine to improve hyperemic flow and symptoms $(2,85,121-124,126,127)$. From the available outcome data for CMD in this population $(115-117,119)$ and, in particular, from the point of view of the clinical decision-making process and treatment options, this scenario was scored as appropriate (score 8).

Scenario 85: Symptomatic patients with positive or negative results of an exercise ECG stress test and exclusion of obstructive CAD by angiography (Score 8 - Appropriate)

Symptomatic patients with HCM and CMD have an increased risk for cardiac arrhythmia and for a worse outcome. In symptomatic patients with positive or negative results of an exercise ECG stress test and exclusion of obstructive CAD by invasive or noninvasive angiography, stress-rest perfusion PET can be performed to identify $\mathrm{CMD}$ as a potential substrate for angina symptoms $(102,105)$. Such symptomatic patients without obstructive CAD but with CMD may benefit from ACE-I, ARB, or ranolazine to improve hyperemic flow and symptoms $(2,3,121-124,126,127)$. From the available outcome data for CMD in this population $(115-117,119)$ and, in particular, from the point of view of the clinical decision-making process and treatment options, this scenario was scored as appropriate (score 8).

Scenario 86: Symptomatic patients with positive or negative results of an exercise ECG stress test and normal SPECT perfusion findings (Score 7 - Appropriate)
Symptomatic patients with HCM and CMD have an increased risk for cardiac arrhythmia and for a worse outcome. In symptomatic patients with positive or negative results of an exercise ECG stress test and normal SPECT perfusion findings, stress-rest perfusion PET can be performed to identify CMD as a potential substrate for angina symptoms $(102,105)$. Such symptomatic patients without obstructive CAD but with CMD may benefit from ACE-I, ARB, or ranolazine to improve hyperemic flow and symptoms $(2,3,121-$ $124,126,127)$. From the available outcome data for CMD in this population $(115-117,119)$ and, in particular, from the point of view of the clinical decision-making process and treatment options, this scenario was scored as appropriate (score 7).

Scenario 87: Asymptomatic patients with positive or negative results of an exercise ECG stress test (Score 3 - Rarely Appropriate)

In asymptomatic patients with HCM and positive or negative results of an exercise ECG stress test, stress-rest perfusion PET can be performed to assess CMD for cardiovascular risk stratification. Nonetheless, no outcome studies are available and there is no diagnostic value with stress-rest myocardial perfusion studies in these patients. In certain high-risk constellations, for example, related to familial risk of sudden cardiac death, stress-rest perfusion PET may be indicated for cardiovascular risk stratification. Thus, this scenario was scored as rarely appropriate (score 3).

Clinical Scenarios 88-107: Symptomatic Patients with Known LV Hypertrophy Related to Arterial Hypertension, Diabetes Mellitus, or Obesity. In symptomatic patients with known LV hypertrophy related to adverse effects of arterial hypertension, diabetes mellitus, or obesity (scenarios 88-107), PET MPI affords concurrent assessment of the presence of hemodynamically obstructive $\mathrm{CAD}$ (perfusion imaging) and microvascular function (MBF quantification) $(2,3,102,104,105)$. In a substantial number of these patients without obstructive CAD (normal perfusion images), PET-determined CMD (reduced hyperemic MBF and MFR) 
may account for reported angina symptoms, which also conveys important prognostic information $(29,39,46,48,114,118,120)$. Thus, apart from the identification of hemodynamically obstructive CAD (stress-related regional perfusion deficit) with PET MPI, it also adds important diagnostic and prognostic information with the concurrent assessment of CMD (MBF quantification). Given the numerous outcome data on CMD in these individuals with cardiovascular risk, with the possibility of intensifying preventive medical care to improve or even normalize CMD with improved control of symptoms $(2,3,121)$ and likely improved outcome, these scenarios were commonly deemed appropriate.

The following scenarios address patients with arterial hypertension.

Scenario 88: Symptomatic patients with positive results of an exercise ECG stress test (Score 9 - Appropriate)

In symptomatic patients with positive results of an exercise ECG stress test, stress-rest perfusion PET can be performed to assess the presence of flow-limiting obstructive CAD and, at the same time, to identify CMD as a potential substrate for angina symptoms $(2,3,102,104,105)$. Positive results of an ECG stress test are nonspecific because stress-related disturbances can be related not only to ischemia, but also to wall thickening or interstitial fibrosis. The advantage of stress-rest perfusion PET is the concurrent evaluation of the presence of obstructive CAD (perfusion deficit) and of CMD. The concurrent assessment of CMD adds diagnostic and prognostic value in these high-risk populations $(3,29,39,48,114,118,120)$. Such symptomatic patients without obstructive CAD but with CMD may also benefit from ACE-I, $\mathrm{ARB}$, or ranolazine to improve hyperemic flow and symptoms $(2,3,121,123,124,126,127)$. From the available outcome data for CMD and from the point of view of the clinical decision-making process and treatment options, this scenario was scored as appropriate (score 9).

Scenario 89: Symptomatic patients with negative results of an exercise ECG stress test (Score 9 - Appropriate)

In symptomatic patients with negative results of an exercise ECG stress test, stress-rest perfusion PET can be performed to assess the presence of flow-limiting obstructive CAD and, at the same time, to identify CMD as a potential substrate for angina symptoms $(2,3,102,104,105)$. Negative results of an ECG stress test may not exclude ischemia or CMD in these patients. The advantage of stress-rest perfusion PET is the concurrent evaluation of the presence of obstructive CAD (perfusion deficit) and of CMD. The concurrent assessment of CMD adds diagnostic and prognostic value in these high-risk populations $(3,29,39,46,48,114,118,120)$. Such symptomatic patients without obstructive CAD but with CMD may also benefit from ACE-I, ARB, or ranolazine to improve hyperemic flow and symptoms $(2,3,121,123,124,126,127)$. From the available outcome data of CMD and the point of view of the clinical decision-making process and treatment options, this scenario was scored as appropriate (score 9).

Scenario 90: Symptomatic patients with positive or negative results of an exercise ECG stress test without evidence of CAD by angiography (Score 9 - Appropriate)

In symptomatic patients with positive or negative results of an exercise ECG stress test without evidence of CAD by angiography, stress-rest perfusion PET can be performed to identify CMD as a potential substrate for angina symptoms $(2,3,102,104,105)$. Such symptomatic patients without obstructive CAD but with CMD may benefit from ACE-I, ARB, or ranolazine to improve hyperemic flow and symptoms $(2,3,121,123,124,126,127)$. From the available outcome data for $\operatorname{CMD}(3,29,39,46,48,114,118,120)$ and the point of view of the clinical decision-making process and treatment options, this scenario was scored as appropriate (score 9).

Scenario 91: Asymptomatic patients with positive results of an exercise ECG stress test (Score 4 - May be Appropriate)

In asymptomatic patients with positive results of an exercise ECG stress test, stress-rest perfusion PET can be performed to assess for macro- and microvascular CAD for cardiovascular risk stratification $(2,3)$. Positive results of an ECG stress test are seen as nonspecific because stress-related disturbances of repolarization can be related not only to ischemia, but also to wall thickening or interstitial fibrosis. No outcome studies are available and there is no diagnostic value with stress-rest myocardial perfusion studies in these asymptomatic patients. Conversely, positive results of an ECG stress test may give rise to concern for silent ischemia that could affect the cardiovascular outcome. From the clinical point of view, stress-rest perfusion PET may be indicated for further diagnostic evaluation and cardiovascular risk stratification in such high-risk populations. Thus, this scenario was scored as may be appropriate (score 4).

Scenario 92: Asymptomatic patients with negative results of an exercise ECG stress test (Score 1 - Rarely Appropriate)

In asymptomatic patients with negative results of an exercise ECG stress test, stress-rest perfusion PET can be performed to assess for macro- and microvascular CAD for cardiovascular risk stratification. Nonetheless, no outcome studies are available and there is no added diagnostic value with stress-rest myocardial perfusion studies in these asymptomatic patients. Thus, this scenario was scored as rarely appropriate (score 1).

Scenario 93: Symptomatic patients with positive or negative results of an exercise ECG stress test and exclusion of coronary atherosclerosis as shown by angiography (Score 2 - Rarely Appropriate)

In symptomatic patients with positive or negative results of an exercise ECG stress test and exclusion of coronary atherosclerosis by angiography, stress-rest perfusion PET can be performed to identify CMD as a potential substrate for angina symptoms. In such patients with completely normal coronary angiogram results, the presence of CMD is very unlikely. Thus, this scenario was scored as rarely appropriate (score 2).

Scenario 94: Symptomatic patients with positive or negative results of an exercise ECG stress test who have atherosclerosis and nonobstructive CAD as shown by angiography (Score $8-$ Appropriate)

In symptomatic patients with positive or negative results of an exercise ECG stress test who have atherosclerosis and nonobstructive CAD as shown by angiography, stress-rest perfusion PET can be performed to identify CMD as a potential substrate for angina symptoms. CMD may be present in up to $60 \%$ of such patients $(102,104,105)$. Symptomatic patients without obstructive CAD but with CMD may benefit from ACE-I, ARB, or ranolazine to improve hyperemic flow and symptoms $(2,3,121,123,124,126,127)$. From the available outcome data for CMD $(29,39,46,48,114,118,120)$ and in particular from the point of view of the clinical decision-making process and treatment options, this scenario was scored as appropriate (score 8). 
The following scenarios address patients with diabetes mellitus.

Scenario 95: Symptomatic patients with positive results of an exercise ECG stress test (Score 8 - Appropriate)

In symptomatic patients with diabetes mellitus and positive results of an exercise ECG stress test, stress-rest perfusion PET can be performed to assess the presence of flow-limiting obstructive $\mathrm{CAD}$ and, at the same time, to identify $\mathrm{CMD}$ as a potential substrate for angina symptoms. Positive results of an ECG stress test are nonspecific because stress-related disturbances can be related not only to ischemia, but also to wall thickening or interstitial fibrosis. The advantage of stress-rest perfusion PET is the concurrent evaluation of the presence of obstructive CAD (perfusion deficit) and of CMD. The concurrent assessment of CMD adds diagnostic and prognostic value in these high-risk populations $(3,48)$. Such symptomatic patients without obstructive CAD but with CMD may also benefit from ACE-I, ARB, or ranolazine to improve hyperemic flow and symptoms $(2,3,121,123,124,126,127)$. From the available outcome data for $\operatorname{CMD}(29,39,46,48,114,118,120)$ and the point of view of the clinical decision-making process and treatment options, this scenario was scored as appropriate (score 8).

Scenario 96: Symptomatic patients with negative results of an exercise ECG stress test (Score 8 - Appropriate)

In symptomatic patients with diabetes mellitus and negative results of an exercise ECG stress test, stress-rest perfusion PET can be performed to assess the presence of flow-limiting obstructive CAD and, at the same time, to identify CMD as a potential substrate for angina symptoms. Negative results of an ECG stress test may not exclude ischemia or CMD in these patients. The advantage of stress-rest perfusion PET is the concurrent evaluation of the presence of obstructive CAD (perfusion deficit) and of CMD. The concurrent assessment of CMD adds diagnostic and prognostic value in these high-risk populations $(3,48)$. Such symptomatic patients without obstructive $\mathrm{CAD}$ but with $\mathrm{CMD}$ may also benefit from ACE-I, ARB, or ranolazine to improve hyperemic flow and symptoms $(2,3,121,123,124,126,127)$. From the available outcome data for CMD $(29,39,46,48,114,118,120)$ and the point of view of the clinical decision-making process and treatment options, this scenario was scored as appropriate (score 8).

Scenario 97: Symptomatic patients with positive or negative results of an exercise ECG stress test who have atherosclerosis and nonobstructive $\mathrm{CAD}$ as shown by angiography (Score 8 - Appropriate)

In symptomatic patients with diabetes mellitus and positive or negative results of an exercise ECG stress test who have atherosclerosis and nonobstructive CAD as shown by angiography, stress-rest perfusion PET can be performed to identify CMD as a potential substrate for angina symptoms. CMD may be present in up to $60 \%$ of such patients $(102,104,105)$. Symptomatic patients without obstructive CAD but with CMD may benefit from ACE-I, $\mathrm{ARB}$, or ranolazine to improve hyperemic flow and symptoms $(2,3,121,123,124,126,127)$. From the available outcome data for CMD $(29,39,46,48,114,118,120)$ and in particular from the point of view of the clinical decision-making process and treatment options, this scenario was scored as appropriate (score 8).

Scenario 98: Symptomatic patients with positive or negative results of an exercise ECG stress test who have normal SPECT perfusion findings (Score 8 - Appropriate)
In symptomatic patients with diabetes mellitus and positive or negative results of an exercise ECG stress test who have normal SPECT perfusion findings, stress-rest perfusion PET can be performed to identify CMD as a potential substrate for angina symptoms. CMD may be present in up to $60 \%$ of such patients $(102,104)$. Symptomatic patients without obstructive CAD but with CMD may benefit from ACE-I, ARB, or ranolazine to improve hyperemic flow and symptoms $(2,3,121,123,124,126,127)$. From the available outcome data for CMD $(29,39,46,48,114,118,120)$ and in particular from the point of view of the clinical decision-making process and treatment options, this scenario was scored as appropriate (score 8).

Scenario 99: Asymptomatic patients with positive results of an exercise ECG stress test (Score 7 - Appropriate)

In asymptomatic patients with diabetes mellitus and positive results of an exercise ECG stress test, stress-rest perfusion PET can be performed to assess for macro- and microvascular CAD for cardiovascular risk stratification $(3,48)$. Positive results of an ECG stress test are nonspecific because stress-related disturbances of the repolarization can be related to ischemia and to interstitial fibrosis. The value of stress-rest myocardial perfusion studies in these asymptomatic but high-risk patients is controversial (128-133). Positive results of an ECG stress test may give rise to concern for silent ischemia that could affect the cardiovascular outcome $(48,130,131)$. In particular, the presence of CMD may predict a worse cardiovascular outcome $(29,39,46,48,114,118,120)$. From the clinical point of view, stress-rest perfusion PET may be indicated for further diagnostic evaluation and cardiovascular risk stratification in such a high-risk population. Thus, this scenario was scored as appropriate (score 7).

Scenario 100: Asymptomatic patients with negative results of an exercise ECG stress test (Score 1 - Rarely Appropriate)

In asymptomatic patients with diabetes mellitus and negative results of an exercise ECG stress test, stress-rest perfusion PET can be performed to assess for macro- and microvascular CAD for cardiovascular risk stratification (128-133). Nonetheless, no specific outcome studies are available and there is no added diagnostic value with stress-rest myocardial perfusion and flow studies in these asymptomatic patients. Thus, this scenario was scored as rarely appropriate (score 1).

The following scenarios address patients with obesity.

Scenario 101: Symptomatic patients with positive results of an exercise ECG stress test (Score 9 - Appropriate)

In symptomatic patients with positive results of an exercise ECG stress test, stress-rest perfusion PET can assess the presence of flow-limiting obstructive $\mathrm{CAD}$ and, at the same time, identify CMD as a potential substrate for angina symptoms. Positive results of an ECG stress test are nonspecific because stress-related disturbances can be related not only to ischemia, but also to wall thickening or interstitial fibrosis. The advantage of stress-rest perfusion PET is the concurrent evaluation of the presence of obstructive CAD (perfusion deficit) with high diagnostic accuracy and of CMD $(29,114,120,134)$. The concurrent assessment of CMD adds diagnostic and prognostic value in these high-risk populations (114). Such symptomatic patients without obstructive CAD but with CMD may also benefit from ACE-I, ARB, or ranolazine to improve hyperemic flow and symptoms $(2,3,121,123,124,126,127)$. From the available outcome data for microvascular disease and, in particular, 
from the point of view of the clinical decision-making process and treatment options, this scenario was scored as appropriate (score 9).

Scenario 102: Symptomatic patients with negative results of an exercise ECG stress test (Score 9 - Appropriate)

In symptomatic patients with negative results of an exercise ECG stress test, stress-rest perfusion PET can be performed to assess the presence of flow-limiting obstructive $\mathrm{CAD}$ and, at the same time, to identify CMD as a potential substrate for angina symptoms. Negative results of an ECG stress test may not exclude ischemia or CMD in these patients. The advantage of stress-rest perfusion PET is the concurrent evaluation of the presence of obstructive CAD (perfusion deficit) with high diagnostic accuracy and of CMD $(29,114,120)$. The concurrent assessment of CMD adds diagnostic and prognostic value in these high-risk populations (114). Such symptomatic patients without obstructive CAD but with CMD may also benefit from ACE-I, ARB, or ranolazine to improve hyperemic flow and symptoms $(2,3,121,123,124,126,127)$. From the available outcome data for CMD and, in particular, from the point of view of the clinical decision-making process and treatment options, this scenario was scored as appropriate (score 9).

Scenario 103: Symptomatic patients with positive or negative results of an exercise ECG stress test who have atherosclerosis and nonobstructive $\mathrm{CAD}$ as shown by angiography (Score 8 - Appropriate)

In symptomatic patients with positive or negative results of an exercise ECG stress test who have atherosclerosis and nonobstructive CAD as shown by angiography, stress-rest perfusion PET can be performed to identify CMD as a potential substrate for angina symptoms. CMD may be present in up to $60 \%$ of such patients $(102,104,105)$. Symptomatic patients without obstructive CAD but with CMD may benefit from ACE-I, ARB, or ranolazine to improve hyperemic flow and symptoms $(2,3,121,123,124,126,127)$. From the available outcome data for $\mathrm{CMD}$ and, in particular, from the point of view of the clinical decision-making process and treatment options, this scenario was scored as appropriate (score 8).

Scenario 104: Symptomatic patients with positive or negative results of an exercise ECG stress test who do not have atherosclerotic CAD as shown by angiography (Score 2 - Rarely Appropriate)

In symptomatic patients with positive or negative results of an exercise ECG stress test who do not have atherosclerotic CAD as shown by angiography, stress-rest perfusion PET can be performed to identify CMD as a potential substrate for angina symptoms. In such patients with completely normal results of a coronary angiogram, the presence of CMD is very unlikely. Thus, this scenario was scored as rarely appropriate (score 2 ).

Scenario 105: Symptomatic patients with positive or negative results of an exercise ECG stress test who have normal SPECT perfusion findings (Score 8 - Appropriate)

In symptomatic patients with positive or negative results of an exercise ECG stress test who have normal SPECT perfusion findings, stress-rest perfusion PET can be performed to identify $\mathrm{CMD}$ as a potential substrate for angina symptoms. The prevalence of CMD may be as high as $60 \%(102,104,105)$. Such symptomatic patients without obstructive CAD but with CMD may benefit from ACE-I, ARB, or ranolazine to improve hyperemic flow and symptoms $(2,3,121,123,124,126,127)$. From the available outcome data for CMD (114) and, in particular, from the point of view of the clinical decision-making process and treatment options, this scenario was scored as appropriate (score 8).

Scenario 106: Asymptomatic patients with positive results of an exercise ECG stress test (Score 5 - May be Appropriate)

In asymptomatic patients with positive results of an exercise ECG stress test, stress-rest perfusion PET can be performed to assess for macro- and microvascular CAD. Positive results of an ECG stress test are nonspecific because stress-related disturbances of repolarization can be related not only to ischemia, but also to wall thickening or interstitial fibrosis. No outcome studies are available and there is no diagnostic value with stress-rest myocardial perfusion studies in these asymptomatic patients. Conversely, positive results of an ECG stress test may give rise to concern for silent ischemia that could affect the cardiovascular outcome $(29,114,120)$. From the clinical point of view, stress-rest perfusion PET may be indicated for further diagnostic evaluation and cardiovascular risk stratification in such a high-risk population (114). Thus, this scenario was scored as may be appropriate (score 5).

Scenario 107: Asymptomatic patients with negative results of an exercise ECG stress test (Score 1 - Rarely Appropriate)

In asymptomatic patients with negative results of an exercise ECG stress test, stress-rest perfusion PET can be performed to assess for macro- and microvascular CAD for cardiovascular risk stratification. Nonetheless, no outcome studies are available and there is no added diagnostic value with stress-rest myocardial perfusion studies in these asymptomatic patients. Thus, this scenario was scored as rarely appropriate (score 1).

Clinical Scenarios 108-114: Postmenopausal Women. In symptomatic postmenopausal women (scenarios 108-114), the application of PET MPI may be critical, as it concurrently determines the presence of hemodynamically obstructive $\mathrm{CAD}$ (perfusion imaging) and microvascular function (MBF quantification) $(2,3,102,104,105)$. Postmenopausal women in the presence of cardiovascular risk and significant reduction in estrogen plasma levels are apt to initiate and rapidly develop CAD or CMD. In particular, there is a high prevalence of CMD in women with recurrent chest pain but nonobstructive CAD. PET MPI offers a comprehensive assessment of the presence of both hemodynamically obstructive CAD and CMD, which conveys important diagnostic and prognostic information $(39,108,113,135-143)$. From the available outcome data for CMD and standard clinical practice regarding the clinical decision-making process and treatment options $(2,3,121)$, these scenarios were commonly deemed appropriate.

Scenario 108: Symptomatic postmenopausal women with positive results of an exercise ECG stress test (Score 9 - Appropriate)

In symptomatic postmenopausal women with positive results of an exercise ECG stress test, stress-rest perfusion PET can be performed to assess the presence of flow-limiting obstructive CAD and, at the same time, to identify CMD as a potential substrate for angina symptoms $(2,85,102,104)$. Positive results of an ECG stress test are nonspecific because stress-related disturbances can be related not only to ischemia, but also to wall thickening or interstitial fibrosis. The advantage of stress-rest perfusion PET is the concurrent evaluation of the presence of obstructive CAD (perfusion deficit) and of CMD. The concurrent assessment of CMD adds important diagnostic and prognostic value in this population $(34,108,113,135-143)$. Such symptomatic patients without 
obstructive CAD but with CMD may also benefit from ACE-I, $\mathrm{ARB}$, or ranolazine to improve hyperemic flow and symptoms $(2,85,121,123,124,126,127,135-139)$. From the available outcome data for CMD and, in particular, from the point of view of the clinical decision-making process and treatment options, this scenario was scored as appropriate (score 9).

Scenario 109: Symptomatic postmenopausal women with negative results of an exercise ECG stress test (Score 9 - Appropriate)

In symptomatic postmenopausal women with negative results of an exercise ECG stress test, stress-rest perfusion PET can be performed to assess the presence of flow-limiting obstructive CAD and, at the same time, to identify CMD as a potential substrate for angina symptoms $(2,85,102,104,105)$. Negative results of an ECG stress test may not exclude ischemia or CMD in these patients. The advantage of stress-rest perfusion PET is the concurrent evaluation of the presence of obstructive CAD (perfusion deficit) and of CMD. The concurrent assessment of CMD adds important diagnostic and prognostic value in this population $(2,85,121,123,124,126,127,135-139)$. Such symptomatic patients without obstructive CAD but with CMD may also benefit from ACE-I, ARB, or ranolazine to improve hyperemic flow and symptoms $(2,85,121,123,124,126,127)$. From the available outcome data of CMD and, in particular, from the point of view of the clinical decision-making process and treatment options, this scenario was scored as appropriate (score 9).

Scenario 110: Symptomatic postmenopausal women with positive or negative results of an exercise ECG stress test and exclusion of coronary atherosclerosis by angiography (Score 1 - Rarely Appropriate)

In symptomatic postmenopausal women with positive or negative results of an exercise ECG stress test and exclusion of coronary atherosclerosis by angiography, stress-rest perfusion PET can be performed to identify CMD as a potential substrate for angina symptoms $(2,85,102,104)$. Because the presence of CMD in such patients without evidence of any atherosclerotic process in the coronary vessels is very unlikely, this scenario was scored as rarely appropriate (score 1).

Scenario 111: Symptomatic postmenopausal women with positive or negative results of an exercise ECG stress test who have atherosclerosis and nonobstructive $\mathrm{CAD}$ as shown by angiography (Score 9 - Appropriate)

In symptomatic postmenopausal women with positive or negative results of an exercise ECG stress test who have atherosclerosis and nonobstructive $\mathrm{CAD}$ as shown by angiography, stress-rest perfusion PET can be performed to identify CMD as a potential substrate for angina symptoms $(2,85,102,104)$. The prevalence of CMD may be as high as $60 \%(102,104,105)$. Such symptomatic patients without obstructive CAD but with CMD may benefit from ACE-I, ARB, or ranolazine to improve hyperemic flow and symptoms $(2,85,121,123,124,126,127)$. From the available outcome data for CMD and, in particular, from the point of view of the clinical decision-making process and treatment options, this scenario was scored as appropriate (score 9).

Scenario 112: Symptomatic postmenopausal women with positive or negative results of an exercise ECG stress test and normal SPECT perfusion findings (Score 9 - Appropriate)

In symptomatic postmenopausal women with positive or negative results of an exercise ECG stress test and normal SPECT perfusion findings, stress-rest perfusion PET can be performed to identify CMD as a potential substrate for angina symptoms $(2,85,102,104)$. The prevalence of CMD may be as high as $60 \%$ $(102,104)$. Such symptomatic patients without obstructive CAD but with CMD may benefit from ACE-I, ARB, or ranolazine to improve hyperemic flow and symptoms $(2,3,121,123,124,126,127)$. From the available outcome data for CMD and, in particular, from the point of view of the clinical decision-making process and treatment options, this scenario was scored as appropriate (score 9).

Scenario 113: Asymptomatic postmenopausal women with positive results of an exercise ECG stress test (Score 5 - May be Appropriate)

In asymptomatic postmenopausal women with positive results of an exercise ECG stress test, stress-rest perfusion PET can be performed to assess for macro- or microvascular CAD $(2,85,102,104)$. Positive results of an ECG stress test are nonspecific because stress-related disturbances of repolarization can be related not only to ischemia, but also to wall thickening or interstitial fibrosis. No outcome studies are available and there is no diagnostic value with stress-rest myocardial perfusion studies in these asymptomatic patients. Conversely, positive results of an ECG stress test may give rise to concern for silent ischemia that could affect the cardiovascular outcome $(108,113,144)$. From the clinical point of view, stress-rest perfusion PET may be indicated for further diagnostic evaluation and cardiovascular risk stratification in such a high-risk population. Thus, this scenario was scored as may be appropriate (score 5).

Scenario 114: Asymptomatic postmenopausal women with negative results of an exercise ECG stress test (Score 1 - Rarely Appropriate)

In asymptomatic postmenopausal women with negative results of an exercise ECG stress test, stress-rest perfusion PET can be performed to assess CMD for cardiovascular risk stratification. Nonetheless, no outcome studies are available and there is no diagnostic value with stress-rest myocardial perfusion studies in these asymptomatic patients. Thus, this scenario was scored as rarely appropriate (score 1).

Clinical Scenarios 115-118: Patients with Syndrome X: No Obstructive CAD but Ongoing Chest Pain Syndrome. In patients with so-called syndrome $\mathrm{X}$, implying nonobstructive $\mathrm{CAD}$ but ongoing chest pain syndrome with recurrent hospitalizations (scenarios 115-118), the application of PET MPI is pivotal in the identification of CMD as a potential substrate for recurrent chest pain $(2,85,102,105,145-148)$ that may be amenable to intensified preventive and vasoactive medical care of cardiovascular risk factors with statins, ACE-I, or ARB. In the absence of cardiovascular risk factors, symptoms may be controlled with calcium antagonists or ranolazine medication $(2,85,121)$. Flow outcome data are limited and controversial in this subset of patients with CMD $(140,143,149,150)$. Previously, the clinical term syndrome $\mathrm{X}$ was reserved for symptomatic patients with normal results of coronary angiograms. The clinical classification of syndrome $\mathrm{X}$ has been extended today to include patients with classic cardiovascular risk factors and nonobstructive CAD. This may explain, at least in part, a certain variability in clinical outcome data in these patients. Overall, the identification of CMD as a potential substrate for recurrent symptoms is central to the diagnosis and clinical decision-making process and for the treatment 
options and medical care for these patients $(2,3,13,121,148)$. Thus, these scenarios were commonly deemed appropriate.

Scenario 115: Patients with ongoing chest pain syndrome and positive results of an exercise ECG stress test who have nonobstructive $\mathrm{CAD}$ as shown by coronary angiography (Score 9 - Appropriate)

In patients with ongoing chest pain syndrome and with positive results of an exercise ECG stress test who have nonobstructive $\mathrm{CAD}$ as shown by coronary angiography, stress-rest perfusion PET can be performed to identify CMD as a potential substrate for angina symptoms $(2,85,102,105,145-148)$. Positive results of an ECG stress test are nonspecific because stress-related disturbances can be related not only to ischemia, but also to wall thickening or interstitial fibrosis. The advantage of stress-rest perfusion PET is the concurrent evaluation of the presence of obstructive CAD (perfusion deficit) and of CMD. The concurrent assessment of CMD adds important diagnostic and prognostic value in this population. The prevalence of CMD may be as high as $60 \%$ $(102,105)$. Such symptomatic patients without obstructive CAD but with CMD may also benefit from ACE-I, ARB, or ranolazine to improve hyperemic flow and symptoms $(2,85,121,123,124,126,127)$. From the available outcome data for CMD and, in particular, from the point of view of the clinical decision-making process and treatment options, this scenario was scored as appropriate (score 9).

Scenario 116: Patients with ongoing chest pain syndrome and negative results of an exercise ECG stress test who have no evidence of obstructive $\mathrm{CAD}$ as shown by coronary angiography (Score 8 - Appropriate)

In patients with ongoing chest pain syndrome and with negative results of an exercise ECG stress test who have no evidence of obstructive CAD as shown by coronary angiography, stress-rest perfusion PET can be performed to identify CMD as a potential substrate for angina symptoms $(2,85,102,105,145-148)$. Negative results of an ECG stress test may not exclude ischemia or CMD in these patients. The advantage of stress-rest perfusion PET is the concurrent evaluation of the presence of obstructive CAD (perfusion deficit) and of CMD. The concurrent assessment of CMD adds important diagnostic and prognostic value in this population. The prevalence of CMD may be as high as $60 \%(102,105)$. Such symptomatic patients without obstructive CAD but with CMD may also benefit from ACE-I, ARB, or ranolazine to improve hyperemic flow and symptoms $(2,85,121,123,124,126,127)$. From the available outcome data for CMD and, in particular, from the point of view of the clinical decision-making process and treatment options, this scenario was scored as appropriate (score 8).

Scenario 117: Patients with ongoing chest pain syndrome, positive results of an exercise ECG stress test, and normal SPECT perfusion findings (Score 9 - Appropriate)

In patients with ongoing chest pain syndrome, positive results of an exercise ECG stress test, and normal SPECT perfusion findings, stress-rest perfusion PET can be performed to identify CMD as a potential substrate for angina symptoms $(2,85,102,105,145-148)$. The prevalence of CMD may be as high as $60 \%(102,105)$. Such symptomatic patients without obstructive CAD but with CMD may benefit from ACE-I, ARB, or ranolazine to improve hyperemic flow and symptoms $(2,85,121,123,124,126,127)$. From the available outcome data for CMD and, in particular, from the clinical point of view, decision-making process, and treatment consequences, this scenario was scored as appropriate (score 9).

Scenario 118: Patients with ongoing chest pain syndrome, negative results of an exercise ECG stress test, and normal SPECT perfusion findings (Score 8 - Appropriate)

In patients with ongoing chest pain syndrome, negative results of an exercise ECG stress test, and normal SPECT perfusion findings, stress-rest perfusion PET can be performed to identify CMD as a potential substrate for angina symptoms (2,85,102,105,145-148). Negative results of an exercise ECG stress test and normal SPECT findings may not exclude CMD. The prevalence of CMD may be as high as $60 \%(102,105)$. Such symptomatic patients without obstructive $\mathrm{CAD}$ but with CMD may benefit from ACE-I, ARB, or ranolazine to improve hyperemic flow and symptoms $(2,85,121,123,124,126,127)$. From the available outcome data of CMD and, in particular, from the point of view of the clinical decision-making process and treatment options, this scenario was scored as appropriate (score 8).

\section{Summary of Recommendations}

Microvascular angina pectoris with underlying CMD has a broad variety of clinical manifestations, as has been outlined by the COVADIS group (102). In the differential diagnosis, it is critical to evaluate or exclude other underlying causes of a chest pain syndrome such as costochondritis, musculoskeletal disturbance, or gastroesophageal reflux. Most of the compiled clinical scenarios imply recurrent chest pain in individuals without evidence of obstructive CAD or the presence of myocardial diseases (e.g., HCM, LV hypertrophy). Thus, the evaluation of CMD with PET perfusion and flow quantification is targeted to a specific clinical scenario that implies that it will convey important diagnostic and prognostic information. Because microvascular angina pectoris constitutes a frequently encountered clinical problem that is amenable to a variety of treatment options $(2,9,85,121)$, PET perfusion, and flow quantification, most of these clinical scenarios have been scored as appropriate. Conversely, PET perfusion and flow quantification should not be applied to asymptomatic individuals or in an uncritical fashion to evaluate for the presence of CMD.

\section{SECTION 8: SPECIFIC POPULATIONS}

\section{Introduction and Background}

This section evaluates the role of PET MPI in specific populations such as those with advanced obesity (body mass index [BMI] $\geq$ $35 \mathrm{~kg} / \mathrm{m}^{2}$ ), congenital heart disease, or familial hypercholesterolemia; women with large breasts or dense breast tissue; young women (age $<45 \mathrm{y}$ ); and young men (age $<40 \mathrm{y}$ ).

\section{Clinical Scenarios and AUC Scores}

Table 8 presents the following clinical scenarios and final AUC scores for the use of PET MPI in specific populations.

Clinical Scenarios 119-133: Patients with Advanced Obesity or Women with Large Breasts or Dense Breast Tissue. In symptomatic patients with advanced obesity (BMI $>35 \mathrm{~kg} / \mathrm{m}^{2}$ ) and women with large breasts or dense breast tissue (scenarios 119-133), PET MPI offers the highest diagnostic accuracy for CAD detection and the lowest radiation exposure in scintigraphic MPI $(2,144,151)$. In addition, the identification of CMD as a potential substrate for recurrent chest pain may be amenable to intensified preventive and vasoactive medical care of cardiovascular risk factors with statins, ACE-I, or ARB $(2,3,121)$. In the absence of cardiovascular risk factors, symptoms may be controlled with calcium antagonists (121) or ranolazine medication $(126,127)$. Recent large-scale PET 
TABLE 8

Clinical Scenarios for the Use of PET MPI in Specific Populations

\begin{tabular}{|c|c|c|c|}
\hline Scenario no. & Description & Appropriateness & Score \\
\hline \multicolumn{4}{|c|}{ Patients with advanced obesity or with large breasts or dense breast tissue } \\
\hline \multicolumn{4}{|c|}{ Patients with advanced obesity (BMI $\left.>35 \mathrm{~m}^{2} / \mathrm{kg}\right)$} \\
\hline 119 & Symptomatic patients with advanced obesity $\left(\mathrm{BMI}>35 \mathrm{~m}^{2} / \mathrm{kg}\right)$ & Appropriate & 9 \\
\hline 120 & $\begin{array}{l}\text { Symptomatic patients with advanced obesity }\left(\mathrm{BMI}>35 \mathrm{~m}^{2} / \mathrm{kg} \text { ) }\right. \\
\text { who have atherosclerosis or nonobstructive CAD as shown by coronary } \\
\text { angiography (invasive or noninvasive) }\end{array}$ & Appropriate & 8 \\
\hline 121 & $\begin{array}{l}\text { Symptomatic patients with advanced obesity }\left(\mathrm{BMI}>35 \mathrm{~m}^{2} / \mathrm{kg} \text { ) }\right. \\
\text { and normal results of coronary angiography (invasive or noninvasive) }\end{array}$ & Appropriate & 7 \\
\hline 122 & $\begin{array}{l}\text { Symptomatic patients with advanced obesity }\left(\mathrm{BMI}>35 \mathrm{~m}^{2} / \mathrm{kg}\right) \\
\text { and equivocal findings on stress-rest myocardial perfusion SPECT }\end{array}$ & Appropriate & 9 \\
\hline 123 & $\begin{array}{l}\text { Asymptomatic patients with advanced obesity }\left(\mathrm{BMI}>35 \mathrm{~m}^{2} / \mathrm{kg}\right) \\
\text { who are at high cardiovascular risk when undergoing noncardiac surgery }\end{array}$ & Appropriate & 7 \\
\hline 124 & $\begin{array}{l}\text { Asymptomatic patients with advanced obesity }\left(\mathrm{BMI}>35 \mathrm{~m}^{2} / \mathrm{kg}\right) \text { who } \\
\text { are at intermediate cardiovascular risk when undergoing noncardiac surgery }\end{array}$ & May be Appropriate & 5 \\
\hline 125 & $\begin{array}{l}\text { Asymptomatic patients with advanced obesity }\left(\mathrm{BMI}>35 \mathrm{~m}^{2} / \mathrm{kg}\right) \text { who } \\
\text { are at low cardiovascular risk when undergoing noncardiac surgery }\end{array}$ & Rarely Appropriate & 2 \\
\hline \multicolumn{4}{|c|}{ Women with large breasts or dense breast tissue that causes attenuation artifacts } \\
\hline 126 & Symptomatic women with large breasts or dense breast tissue & Appropriate & 8 \\
\hline 127 & $\begin{array}{l}\text { Symptomatic women with large breasts or dense breast tissue who have } \\
\text { atherosclerosis or nonobstructive CAD as shown by angiography (invasive } \\
\text { or noninvasive) }\end{array}$ & Appropriate & 8 \\
\hline 128 & $\begin{array}{l}\text { Symptomatic women with large breasts or dense breast tissue and normal } \\
\text { results of coronary angiography (invasive or noninvasive) }\end{array}$ & Appropriate & 7 \\
\hline 129 & $\begin{array}{l}\text { Symptomatic women with large breasts or dense breast tissue and normal } \\
\text { results of stress-rest myocardial perfusion SPECT }\end{array}$ & Appropriate & 7 \\
\hline 130 & $\begin{array}{l}\text { Symptomatic women with large breasts or dense breast tissue and equivocal } \\
\text { findings on stress-rest myocardial perfusion SPECT }\end{array}$ & Appropriate & 9 \\
\hline 131 & $\begin{array}{l}\text { Asymptomatic women with large breasts or dense breast tissue at high } \\
\text { risk when undergoing noncardiac surgery }\end{array}$ & Appropriate & 7 \\
\hline 132 & $\begin{array}{l}\text { Asymptomatic women with large breasts or dense breast tissue at } \\
\text { intermediate risk when undergoing noncardiac surgery }\end{array}$ & May be Appropriate & 5 \\
\hline 133 & $\begin{array}{l}\text { Asymptomatic women with large breasts or dense breast tissue at low } \\
\text { risk when undergoing noncardiac surgery }\end{array}$ & Rarely Appropriate & 2 \\
\hline \multicolumn{4}{|c|}{ Symptomatic young women and young men } \\
\hline \multicolumn{4}{|c|}{ Symptomatic young women (age < 45 y) } \\
\hline 134 & $\begin{array}{l}\text { Symptomatic young women (age }<45 \text { y) with positive or negative results of } \\
\text { an exercise ECG stress test or negative results of stress imaging testing with } \\
\text { SPECT or echocardiography, but persistent chest pain syndrome }\end{array}$ & Appropriate & 8 \\
\hline 135 & $\begin{array}{l}\text { Symptomatic young women (age }<45 \text { y) with positive or negative results of an } \\
\text { exercise ECG stress test, who have atherosclerosis or nonobstructive CAD as } \\
\text { shown by angiography, but persistent chest pain syndrome }\end{array}$ & Appropriate & 8 \\
\hline 136 & $\begin{array}{l}\text { Symptomatic young women (age }<45 \text { y) with positive or negative results of an } \\
\text { exercise ECG stress test and normal results of coronary angiography, but } \\
\text { persistent chest pain syndrome }\end{array}$ & Appropriate & 7 \\
\hline 137 & $\begin{array}{l}\text { Symptomatic young women with positive or negative results of an exercise } \\
\text { ECG stress test and normal SPECT perfusion findings, but persistent chest } \\
\text { pain syndrome }\end{array}$ & Appropriate & 7 \\
\hline \multicolumn{4}{|c|}{ Symptomatic young men (age < 40 y) } \\
\hline 138 & $\begin{array}{l}\text { Symptomatic young men (age }<40 \mathrm{y} \text { ) with negative results of stress-rest imaging } \\
\text { testing with SPECT or echocardiography, but persistent chest pain syndrome }\end{array}$ & Appropriate & 8 \\
\hline
\end{tabular}




\begin{tabular}{|c|c|c|c|}
\hline Scenario no. & Description & Appropriateness & Score \\
\hline 139 & $\begin{array}{l}\text { Symptomatic young men (age }<40 \text { y) with positive or negative results of an } \\
\text { exercise ECG stress test, who have atherosclerosis, nonobstructive CAD, and } \\
\text { persistent chest pain syndrome }\end{array}$ & Appropriate & 8 \\
\hline 140 & $\begin{array}{l}\text { Symptomatic young men (age }<40 \mathrm{y} \text { ) with positive or negative results of an } \\
\text { exercise ECG stress test and normal results on a coronary angiogram, but } \\
\text { persistent chest pain syndrome }\end{array}$ & Appropriate & 7 \\
\hline \multicolumn{4}{|c|}{ Children and adolescents with congenital heart disease } \\
\hline 141 & $\begin{array}{l}\text { In children and adolescents with congenital heart disease that could compromise } \\
\text { the blood flow supply to the heart, exercise echocardiography, because of the } \\
\text { absence of any radiation exposure, should be given preference. If exercise } \\
\text { echocardiography is not feasible, then exercise and rest perfusion SPECT is } \\
\text { an option. If the patient cannot exercise or the congenital heart disease may } \\
\text { compromise the blood flow supply independent of exercise stress, then } \\
\text { stress-only or stress-rest perfusion PET is a viable option because of its } \\
\text { low radiation exposure. }\end{array}$ & Appropriate & 8 \\
\hline \multicolumn{4}{|c|}{ Patients with familial hypercholesterolemia } \\
\hline 142 & $\begin{array}{l}\text { Symptomatic individuals with familial hypercholesterolemia with negative } \\
\text { stress-rest imaging findings, but persistent chest pain syndrome }\end{array}$ & Appropriate & 7 \\
\hline 143 & $\begin{array}{l}\text { Symptomatic individuals with familial hypercholesterolemia with positive or } \\
\text { negative results of an exercise ECG stress test, who have atherosclerosis } \\
\text { and nonobstructive CAD, but persistent chest pain }\end{array}$ & Appropriate & 8 \\
\hline 144 & $\begin{array}{l}\text { Symptomatic individuals with familial hypercholesterolemia with positive or } \\
\text { negative results of an exercise ECG stress test and normal results on a } \\
\text { coronary angiogram, but persistent chest pain }\end{array}$ & Appropriate & 7 \\
\hline 145 & $\begin{array}{l}\text { Symptomatic individuals with familial hypercholesterolemia with positive or } \\
\text { negative results of an exercise ECG stress test who have no coronary artery } \\
\text { calcifications on noncontrast CCT }\end{array}$ & Appropriate & 7 \\
\hline 146 & $\begin{array}{l}\text { Symptomatic individuals with familial hypercholesterolemia and positive or } \\
\text { negative results of an exercise ECG stress test who have coronary artery } \\
\text { calcifications on noncontrast CCT }\end{array}$ & Appropriate & 8 \\
\hline 147 & $\begin{array}{l}\text { Asymptomatic individuals with familial hypercholesterolemia with positive or } \\
\text { negative results of an exercise ECG stress test }\end{array}$ & Rarely Appropriate & 2 \\
\hline \multicolumn{4}{|c|}{ Symptomatic patients with CAD with known left main or multivessel disease } \\
\hline 148 & Symptomatic patients with known left main or multivessel disease as shown by ICA & Appropriate & 9 \\
\hline 149 & $\begin{array}{l}\text { Symptomatic patients with CAD in the left main segment or in all } 3 \text { vessels as } \\
\text { shown by noninvasive CTA }\end{array}$ & Appropriate & 9 \\
\hline 150 & $\begin{array}{l}\text { Symptomatic patients with coronary artery calcifications in the left main segment } \\
\text { or in all } 3 \text { vessels as shown by noncontrast CCT }\end{array}$ & Appropriate & 9 \\
\hline \multicolumn{4}{|c|}{ Asymptomatic patients with CAD with known left main or multivessel disease } \\
\hline 151 & $\begin{array}{l}\text { Asymptomatic patients with known left main or multivessel disease as shown } \\
\text { by coronary angiography }\end{array}$ & May be Appropriate & 6 \\
\hline 152 & $\begin{array}{l}\text { Asymptomatic patients with pronounced coronary calcifications in the left main } \\
\text { or in all } 3 \text { main vessels as shown by noncontrast CCT }\end{array}$ & Appropriate & 7 \\
\hline 153 & $\begin{array}{l}\text { Asymptomatic patients with mild coronary calcifications in the left main or } \\
\text { in all } 3 \text { main vessels as shown by noncontrast CCT }\end{array}$ & Rarely Appropriate & 3 \\
\hline \multicolumn{4}{|c|}{ Patients who have undergone CABG } \\
\hline 154 & $\begin{array}{l}\text { Symptomatic patients after undergoing CABG: stress-rest perfusion PET } \\
\text { if exercise SPECT cannot be performed }\end{array}$ & Appropriate & 8 \\
\hline 155 & $\begin{array}{l}\text { Symptomatic patients after undergoing CABG: stress-rest perfusion PET } \\
\text { if exercise SPECT is equivocal }\end{array}$ & Appropriate & 9 \\
\hline 156 & Asymptomatic patients within 5 y of undergoing CABG & Rarely Appropriate & 2 \\
\hline 157 & Asymptomatic patients $\geq 5$ y after undergoing CABG & May be Appropriate & 6 \\
\hline
\end{tabular}




\begin{tabular}{|c|c|c|c|}
\hline Scenario no. & Description & Appropriateness & Score \\
\hline \multicolumn{4}{|c|}{ Patients who have undergone $\mathrm{PCI}$ in multivessel disease } \\
\hline 158 & $\begin{array}{l}\text { Symptomatic patients with known multivessel disease who have undergone } \mathrm{PCl} \text {, } \\
\text { in whom exercise SPECT cannot be performed }\end{array}$ & Appropriate & 8 \\
\hline 159 & $\begin{array}{l}\text { Symptomatic patients with known multivessel disease who have undergone } \mathrm{PCl} \\
\text { and who have equivocal exercise SPECT results }\end{array}$ & Appropriate & 9 \\
\hline 160 & $\begin{array}{l}\text { Asymptomatic patients within } 2 \text { y of undergoing PCI, in whom the diagnostic } \\
\text { yield of stress-rest perfusion PET would be low or nonexistent }\end{array}$ & Rarely Appropriate & 2 \\
\hline 161 & Asymptomatic patients $\geq 2$ y after undergoing $\mathrm{PCl}$ & May be Appropriate & 6 \\
\hline \multicolumn{4}{|c|}{ Use of rest or stress-rest perfusion in patients in conjunction with ${ }^{18} \mathrm{~F}-\mathrm{FDG}$ PET to assess cardiac sarcoidosis } \\
\hline 162 & $\begin{array}{l}\text { Patients with suspected cardiac sarcoidosis: rest perfusion PET as an integral } \\
\text { part of the }{ }^{18} \mathrm{~F}-\mathrm{FDG} \text { PET protocol in the detection and characterization of } \\
\text { cardiac sarcoidosis }\end{array}$ & Appropriate & 8 \\
\hline 163 & $\begin{array}{l}\text { Patients with suspected cardiac sarcoidosis and concurrent chest pain symptoms } \\
\text { or dyspnea, who are undergoing rest perfusion and the }{ }^{18} \text { F-FDG PET protocol: } \\
\text { addition of stress perfusion PET }\end{array}$ & Appropriate & 8 \\
\hline \multicolumn{4}{|c|}{ Use of rest or stress-rest perfusion in patients in conjunction with ${ }^{18} \mathrm{~F}-\mathrm{FDG}$ PET to assess myocardial viability } \\
\hline 164 & $\begin{array}{l}\text { Patients with assessment of myocardial viability in ischemic cardiomyopathy: rest } \\
\text { or stress perfusion PET as an integral part of the }{ }^{18} \mathrm{~F}-\mathrm{FDG} \text { PET protocol in the } \\
\text { detection and characterization of myocardial viability }\end{array}$ & Appropriate & 9 \\
\hline \multicolumn{4}{|c|}{$\begin{array}{l}\text { Symptomatic patients who are apparent nonresponders to pharmacologic vasodilation with a need to change to } \\
\text { dobutamine stress testing }\end{array}$} \\
\hline 165 & $\begin{array}{l}\text { Symptomatic patients with an intermediate probability of CAD who are undergoing } \\
\text { functional testing with myocardial perfusion SPECT or PET during pharmacologic } \\
\text { stimulation and rest: repeat imaging test with PET but with dobutamine } \\
\text { stimulation if there is no hemodynamic response (change in arterial blood } \\
\text { pressure or no heart rate increase) during pharmacologic vasodilator stress }\end{array}$ & Rarely Appropriate & 3 \\
\hline \multicolumn{4}{|c|}{ Symptomatic patients with transplant vasculopathy } \\
\hline 166 & Symptomatic patients or patients with suspected cardiac graft rejection & Appropriate & 8 \\
\hline \multicolumn{4}{|c|}{ Symptomatic patients with vasculitis and arteritis (Kawasaki and Takayasu disease) } \\
\hline 167 & Symptomatic patients with suspected vasculitis and arteritis & Appropriate & 8 \\
\hline
\end{tabular}

MPI investigations in obesity $(29,39,114,120)$ also emphasize incremental adverse outcomes in the presence of CMD in obesity. Given the high diagnostic accuracy of CAD detection, the relatively low radiation exposure in these 2 specific populations, the concurrent assessment of CMD that conveys important diagnostic and prognostic information, and the possibility of intensifying preventive medical care to improve or even normalize CMD with improved control of symptoms and likely improved outcome, these scenarios were commonly deemed appropriate.

The following scenarios address advanced obesity (BMI $>35$ $\mathrm{kg} / \mathrm{m}^{2}$ ).

Scenario 119: Symptomatic patients with advanced obesity $($ BMI $>35 \mathrm{~kg} / \mathrm{m} 2)($ Score 9 - Appropriate)

In symptomatic patients with advanced obesity $\left(\mathrm{BMI}>35 \mathrm{~kg} / \mathrm{m}^{2}\right)$, stress-rest PET MPI can be performed to assess macro- and microvascular CAD disease. The reasoning behind this is that it has the highest diagnostic accuracy in the detection of flow-limiting CAD among all noninvasive imaging modalities $(134,144,151)$. In addition, the concurrent assessment of MFR provides important information about the presence of CMD as a potential substrate for angina symptoms $(2,3,102,104)$. Such symptomatic patients without obstructive CAD but with CMD may benefit from ACE-I, ARB, or ranolazine to improve hyperemic flow and symptoms $(2,3,121,123,124,126,127)$.
From the available outcome data for CMD $(29,39,114,120)$ and, in particular, from the point of view of the clinical decision-making process and treatment consequences, this scenario was scored as appropriate (score 9).

Scenario 120: Symptomatic patients with advanced obesity (BMI $>35 \mathrm{~kg} / \mathrm{m} 2$ ) who have atherosclerosis or nonobstructive $\mathrm{CAD}$ as shown by coronary angiography (invasive or noninvasive) (Score 8 - Appropriate)

In symptomatic patients with advanced obesity (BMI $>35 \mathrm{~kg} / \mathrm{m}^{2}$ ) who have atherosclerosis or nonobstructive $\mathrm{CAD}$ as shown by coronary angiography (invasive or noninvasive), stress-rest PET MPI can be performed to identify CMD as a potential substrate for angina symptoms $(2,3,102,104)$. The prevalence of CMD may be as high as $60 \%$ $(102,104)$. Such symptomatic patients without obstructive CAD but with CMD may benefit from ACE-I, ARB, or ranolazine to improve hyperemic flow and symptoms $(2,3,121,123,124,126,127)$. From the available outcome data for $\operatorname{CMD}(39,114)$ and, in particular, from the point of view of the clinical decision-making process and treatment consequences, this scenario was scored as appropriate (score 8).

Scenario 121: Symptomatic patients with advanced obesity (BMI $>35 \mathrm{~kg} / \mathrm{m} 2$ ) and normal coronary angiography results (invasive or noninvasive) (Score 7 - Appropriate) 
In symptomatic patients with advanced obesity (BMI $>35 \mathrm{~kg} / \mathrm{m}^{2}$ ) and normal coronary angiography results (invasive or noninvasive), stress-rest PET MPI can be performed to identify CMD as a potential substrate for angina symptoms $(2,3,102,104)$. The prevalence of CMD in such patients appears to be marked $(102,104)$, even without evidence of any atherosclerotic process in the coronary vessels, which conveys important prognostic information (114). From the available outcome data for CMD $(39,114)$, the high diagnostic accuracy of PET perfusion imaging in this specific population $(29,144)$, and the point of view of the clinical decision-making process and treatment consequences, this scenario was scored as appropriate (score 7).

Scenario 122: Symptomatic patients with advanced obesity (BMI $>35 \mathrm{~kg} / \mathrm{m} 2$ ) and equivocal findings on stress-rest myocardial perfusion SPECT (Score 9 - Appropriate)

In symptomatic patients with advanced obesity (BMI $>35 \mathrm{~kg} / \mathrm{m}^{2}$ ) and equivocal findings on stress-rest myocardial perfusion SPECT, an additional stress-rest PET MPI can be performed for definitive evaluation of the presence or absence of obstructive CAD because PET has the highest diagnostic accuracy in the detection of flowlimiting CAD in this specific population $(29,134,144)$. The concurrent assessment of MFR provides important information about the presence of CMD as a potential substrate for angina symptoms $(2,3,102,104)$. From the available outcome data $(39,114)$, clinical decision-making process, and treatment consequences, this scenario was scored as appropriate (score 9).

Scenario 123: Asymptomatic patients with advanced obesity $(\mathrm{BMI}>35 \mathrm{~kg} / \mathrm{m} 2)$ who are at high cardiovascular risk when undergoing noncardiac surgery (Score 7 - Appropriate)

In asymptomatic patients with advanced obesity (BMI $>35 \mathrm{~kg} / \mathrm{m}^{2}$ ) who are at high cardiovascular risk when undergoing noncardiac surgery, stress-rest cardiac perfusion PET can be seen as the best noninvasive perfusion imaging modality for preoperative cardiac risk stratification. From the available outcome data $(39,114)$, standard clinical practice (28), and treatment consequences, this scenario was scored as appropriate (score 7).

Scenario 124: Asymptomatic patients with advanced obesity (BMI $>35 \mathrm{~kg} / \mathrm{m} 2$ ) who are at intermediate cardiovascular risk when undergoing noncardiac surgery (Score 5 - May be Appropriate)

In asymptomatic patients with advanced obesity (BMI $>35 \mathrm{~kg} /$ $\mathrm{m}^{2}$ ) who are at intermediate cardiovascular risk when undergoing noncardiac surgery, stress-rest cardiac perfusion PET can be seen as the best noninvasive perfusion imaging modality for preoperative cardiac risk stratification. From the available outcome data $(39,114)$, standard clinical practice $(28)$, and treatment consequences, this scenario was scored as may be appropriate (score 5).

Scenario 125: In asymptomatic patients with advanced obesity $(\mathrm{BMI}>35 \mathrm{~kg} / \mathrm{m} 2)$ who are at low cardiovascular risk when undergoing noncardiac surgery (Score 2 - Rarely Appropriate)

In asymptomatic patients with advanced obesity who are at low cardiovascular risk when undergoing noncardiac surgery, stressrest cardiac perfusion PET can be seen as the best noninvasive perfusion imaging modality for preoperative cardiac risk stratification but is rarely appropriate (score 2 ).

The following scenarios address women with large breasts or with dense breast tissue that causes attenuation artifacts.
Scenario 126: Symptomatic women with large breasts or dense breast tissue (Score 8 - Appropriate)

In symptomatic women with large breasts or dense breast tissue, stress-rest PET MPI can be performed, as it has the highest diagnostic accuracy in the detection of flow-limiting CAD among all noninvasive imaging modalities and the lowest radiation exposure, in particular for the breast $(29,144)$. In addition, the concurrent assessment of MFR provides important information about the presence of $\mathrm{CMD}$ as a potential substrate for angina symptoms. From the available outcome data $(39,108,113,135-143)$, clinical decision-making process, and treatment consequences $(2,3,121,123,124,126,127)$, this scenario was scored as appropriate (score 8).

Scenario 127: Symptomatic women with large breasts or dense breast tissue who have atherosclerosis or nonobstructive $\mathrm{CAD}$ as shown by angiography (invasive or noninvasive) (Score 8 - Appropriate)

In symptomatic women with large breasts or dense breast tissue who have atherosclerosis or nonobstructive CAD as shown by angiography (invasive or noninvasive), stress-rest PET MPI and PET/CT can be performed, as the concurrent assessment of MFR provides important information about the presence of CMD as a potential substrate for angina symptoms $(2,3,102,104)$. The prevalence of CMD may be as high as $60 \%(102,104)$. Such symptomatic patients without obstructive CAD but with CMD may benefit from ACE-I, ARB, or ranolazine to improve hyperemic flow and symptoms $(2,3,121,123,124,126,127)$. From the available outcome data of CMD $(39,108,113,135-143)$ and in view of the clinical decision-making process and treatment consequences, this scenario was scored as appropriate (score 8).

Scenario 128: Symptomatic women with large breasts or dense breast tissue and normal coronary angiography results (invasive or noninvasive) (Score 7 - Appropriate)

In symptomatic women with large breasts or dense breast tissue and normal coronary angiography results (invasive or noninvasive), stress-rest PET MPI and PET/CT can be performed, as the concurrent assessment of MFR provides important information about the presence of CMD as a potential substrate for angina symptoms $(2,3,102,104)$. Microvascular disease in postmenopausal women is common, even with completely normal coronary angiogram results. Such symptomatic patients with normal coronary angiogram results but with CMD may benefit from ACE-I, $\mathrm{ARB}$, or ranolazine to improve hyperemic flow and symptoms $(2,3,121,123,124,126,127)$. From the available outcome data for CMD $(39,108,113,135-143)$ and in view of the clinical decisionmaking process and treatment consequences, this scenario was scored as appropriate (score 7).

Scenario 129: Symptomatic women with large breasts or dense breast tissue who have normal results on stress-rest myocardial perfusion SPECT (Score 7 - Appropriate)

In symptomatic women with large breasts or dense breast tissue who have normal results on stress-rest myocardial perfusion SPECT, an additional stress-rest PET MPI/CT can be performed, as the concurrent assessment of MFR provides important information about the presence of CMD as a potential substrate for angina symptoms $(2,3,102,104)$. Normal results on perfusion SPECT cannot rule out CMD. Given the outcome data $(39,108,113,135-143)$, clinical decision-making 
process, and treatment options $(2,3,121,123,124,126,127)$, this scenario was scored as appropriate (score 7).

Scenario 130: Symptomatic women with large breasts or dense breast tissue who have equivocal findings on stress-rest myocardial perfusion SPECT (Score 9 - Appropriate)

In symptomatic women with large breasts or dense breast tissue who have equivocal findings on stress-rest myocardial perfusion SPECT, an additional stress-rest PET MPI can be performed for definitive evaluation of the presence or absence of obstructive CAD because PET has the highest diagnostic accuracy in the detection of flow-limiting CAD in such conditions $(29,144)$. The concurrent assessment of MFR provides important information about the presence of CMD as a potential substrate for angina symptoms $(2,3,102,104)$. Given the outcome data $(39,108,113,135-143)$, clinical decisionmaking process, and treatment options $(2,3,121,123,124,126,127)$, this scenario was scored as appropriate (score 9).

Scenario 131: Asymptomatic women with large breasts or dense breast tissue who are at high cardiovascular risk when undergoing noncardiac surgery (Score 7 - Appropriate)

In asymptomatic women with large breasts or dense breast tissue who are at high cardiovascular risk when undergoing noncardiac surgery, stress-rest cardiac perfusion PET can be seen as the best noninvasive perfusion imaging modality for preoperative cardiac risk stratification. From the available outcome data $(39,108,113,135-143)$, standard clinical practice (28), and treatment consequences, this scenario was scored as appropriate (score 7).

Scenario 132: Asymptomatic women with large breasts or dense breast tissue who are at intermediate cardiovascular risk when undergoing noncardiac surgery (Score 5 - May be Appropriate)

In asymptomatic women with large breasts or dense breast tissue who are at intermediate cardiovascular risk when undergoing noncardiac surgery, stress-rest cardiac perfusion PET can be seen as the best noninvasive perfusion imaging modality for preoperative cardiac risk stratification. From the available outcome data $(39,108,113,135-$ 143), standard clinical practice (28), and treatment consequences, this scenario was scored as may be appropriate (score 5).

Scenario 133: Asymptomatic women with large breasts or dense breast tissue who are at low cardiovascular risk when undergoing noncardiac surgery (Score 2 - Rarely Appropriate)

In asymptomatic women with large breasts or dense breast tissue who are at low cardiovascular risk when undergoing noncardiac surgery, stress-rest cardiac perfusion PET can be seen as the best noninvasive perfusion imaging modality for preoperative cardiac risk stratification. From the available outcome data $(39,108,113,135-143)$, standard clinical practice (28), and treatment consequences, this scenario was scored as rarely appropriate (score 2).

Clinical Scenarios 134-140: Symptomatic Young Women and Young Men. In symptomatic young women (age $<45$ y) and young men (age $<40$ y) (scenarios 134-140), the application of PET MPI may be seen as helpful for the identification of CMD in the absence of obstructive and flow-limiting CAD. In such patients who have undergone a traditional exercise ECG stress test in conjunction with a SPECT or echocardiography stress test or noninvasive CTA without evidence of obstructive CAD, PET MPI seems to be appropriate for the identification of CMD as a potential substrate for recurrent chest pain that may be amenable to intensified preventive and vasoactive medical care of cardiovascular risk factors with statins, ACE-I, or ARB $(2,3,121)$. In the absence of cardiovascular risk factors, symptoms may be controlled with calcium antagonists (121) or ranolazine $(125,127)$. Given the outcome data for CMD about cardiovascular risk in individuals at this younger age $(3,29,39,46,48,114,118,120)$, the relatively low radiation burden with PET MPI, and the possibility of intensifying preventive medical care to improve or even normalize CMD with improved control of symptoms and likely improved outcome, these scenarios were commonly deemed appropriate but only when obstructive CAD has been excluded. A specific assessment of CMD appears to be needed in the appropriate clinical context.

The following scenarios address young women (age $<45 \mathrm{y}$ ).

Scenario 134: Symptomatic young women (age $<45$ y) with positive or negative results of an exercise ECG stress test or negative results of stress imaging testing with SPECT or echocardiography, but persistent chest pain syndrome (Score 8 - Appropriate)

In symptomatic young women (age $<45 \mathrm{y}$ ) with positive or negative results of an exercise ECG stress test or negative results of stress imaging testing with SPECT or echocardiography but persistent chest pain syndrome, stress-rest MPI is seen as the first choice for the evaluation of CAD because PET is commonly done with pharmacologic vasodilator stress or, in rare instances, with dobutamine stress provocation. In the case of normal perfusion findings on SPECT images and persistent chest pain syndrome, an additional stress-rest PET MPI can be done to assess MFR and thus the presence of CMD as a potential substrate for angina symptoms $(2,3,102,104)$. Such symptomatic patients without obstructive CAD but with CMD may benefit from ACE-I, ARB, or ranolazine to improve hyperemic flow and symptoms. From the available outcome data for CMD $(39,108,113,135-143)$, clinical decision-making process, and treatment consequences $(2,3,121,123,124,126,127)$, this scenario was scored as appropriate (score 8 ).

Scenario 135: Symptomatic young women (age $<45$ y) with positive or negative results of an exercise ECG stress test, who have atherosclerosis or nonobstructive CAD as shown by angiography, but persistent chest pain syndrome (Score 8 - Appropriate)

In symptomatic young women (age $<45 \mathrm{y}$ ) with positive or negative results of an exercise ECG stress test, who have atherosclerosis or nonobstructive CAD as shown by angiography but persistent chest pain syndrome, stress-rest PET MPI can be performed to assess MFR and thus the presence of CMD as a potential substrate for angina symptoms $(2,3,102,104)$. Such symptomatic patients without evidence of CAD but with CMD may benefit from ACE-I, ARB, or ranolazine to improve hyperemic flow and symptoms $(2,3,121,123,124,126,127)$. From the available outcome data for $\operatorname{CMD}(39,108,113,135-143)$, clinical decision-making process, and treatment consequences, this scenario was scored as appropriate (score 8).

Scenario 136: Symptomatic young women (age $<45$ y) with positive or negative results of an exercise ECG stress test and normal coronary angiography results, but persistent chest pain syndrome (Score 7 - Appropriate)

In symptomatic young women (age $<45 \mathrm{y}$ ) with positive or negative results of an exercise ECG stress test and normal coronary angiography results but persistent chest pain syndrome, stress-rest 
PET MPI can be performed to assess MFR and thus the presence of CMD as a potential substrate for angina symptoms $(2,3,102,104)$. Despite normal coronary angiogram results, CMD in this population is frequently encountered $(102,104)$. Such symptomatic patients may benefit from ACE-I, ARB, or ranolazine to improve hyperemic flow and symptoms $(2,3,121,123,124,126,127)$. From the available outcome data for CMD $(39,108,113,135-143)$, clinical decisionmaking process, and treatment consequences, this scenario was scored as appropriate (score 7).

Scenario 137: Symptomatic young women (age $<45$ y) with positive or negative results of an exercise ECG stress test and normal SPECT perfusion findings, but persistent chest pain syndrome (Score 8 - Appropriate)

In symptomatic young women (age $<45$ y) with positive or negative results of an exercise ECG stress test and normal SPECT perfusion findings but persistent chest pain syndrome, stress-rest perfusion PET can be performed to identify CMD as a potential substrate for angina symptoms $(2,3,102,104)$. Findings of the exercise ECG stress test are nonspecific and normal perfusion findings with SPECT do not rule out CMD. Such symptomatic patients without obstructive CAD but with CMD may benefit from ACE-I, ARB, or ranolazine to improve hyperemic flow and symptoms $(2,3,121,123,124,126,127)$. From the available outcome data for CMD $(39,108,113,135-143)$ and in view of the clinical decision-making process and treatment consequences, this scenario was scored as appropriate (score 8).

The following scenarios address young men (age $<40 \mathrm{y}$ ).

Scenario 138: Symptomatic young men (age $<40$ y) with negative results of stress-rest imaging testing with SPECT or echocardiography, but persistent chest pain syndrome (Score 8 - Appropriate)

In symptomatic young men (age $<40$ y) with negative results of stress-rest imaging testing with SPECT or echocardiography but persistent chest pain syndrome, an additional stress-rest PET MPI is seen as first choice for the evaluation of CAD. An additional stress-rest PET MPI to assess MFR enables the identification of CMD that may reflect a functional substrate for chest pain amenable to medical treatment $(2,3,102,104)$. Such symptomatic patients without obstructive CAD but with CMD may benefit from ACE-I, ARB, or ranolazine to improve hyperemic flow and symptoms $(2,3,121,123,124,126,127)$. From the available outcome data for CMD $(29,39,46,48,114,118,120)$ and in view of the clinical decision-making process and treatment consequences, this scenario was scored as appropriate (score 8).

Scenario 139: Symptomatic young men (age $<40$ y) with positive or negative results of an exercise ECG stress test, who have atherosclerosis, nonobstructive $\mathrm{CAD}$, and persistent chest pain syndrome (Score 8 - Appropriate)

In symptomatic young men (age $<40 \mathrm{y}$ ) with positive or negative results of an exercise ECG stress test, who have atherosclerosis, nonobstructive $\mathrm{CAD}$, and persistent chest pain syndrome, stressrest PET MPI can be performed to assess MFR and thus the presence of $\mathrm{CMD}$ as a potential substrate for angina symptoms $(2,3,102,104)$. The prevalence of CMD may be as high as $60 \%$ (102). Such symptomatic patients without obstructive CAD but with CMD may benefit from ACE-I, ARB, or ranolazine to improve hyperemic flow and symptoms $(2,3,121,123,124,126,127)$. From the available outcome data for $\operatorname{CMD}(29,39,46,48,114,118,120)$, and in view of the clinical decision-making process and treatment consequences, this scenario was scored as appropriate (score 8).

Scenario 140: Symptomatic young men (age $<40$ y) with positive or negative results of an exercise ECG stress test and normal results on a coronary angiogram, but persistent chest pain syndrome (Score 7 - Appropriate)

In symptomatic young men (age $<40 \mathrm{y}$ ) with positive or negative results of an exercise ECG stress test and normal coronary angiogram results, but persistent chest pain syndrome, stress-rest PET MPI can be performed to assess MFR and thus the presence of CMD as a potential substrate for angina symptoms $(2,85,102,104,105)$. Despite normal coronary angiogram results (102), CMD may be frequently encountered in this population. Such symptomatic patients without any evidence of CAD but with CMD may benefit from ACE-I, $\mathrm{ARB}$, or ranolazine to improve hyperemic flow and symptoms $(2,3,121,123,124,126,127)$. From the available outcome data for CMD $(29,39,46,48,114,118,120)$, and in view of the clinical decision-making process and treatment consequences, this scenario was scored as appropriate (score 7).

Clinical Scenario 141: Children and Adolescents with Congenital Heart Disease. In symptomatic children and adolescents patients with congenital heart disease (scenario 141), exercise stress testing with imaging is seen as a first option to verify whether the congenital heart disease may affect blood flow to the heart during daily activities and exercise (152-154). If the patient cannot exercise or the congenital heart disease may compromise the blood flow supply independent of exercise stress, then stress-only or stress-rest perfusion PET was seen as a viable option because of its low radiation exposure, and the indication for PET MPI was deemed appropriate.

Scenario 141: Symptomatic children and adolescents with congenital heart disease (Score 8 - Appropriate)

In symptomatic children and adolescents with congenital heart disease that could compromise the blood flow supply to the heart, exercise echocardiography should be given preference because of the absence of any radiation exposure. If exercise echocardiography is not feasible, then exercise and rest perfusion SPECT is an option. If the patient cannot exercise or the congenital heart disease may compromise the blood flow supply independent of exercise stress, then stress-only or stress-rest perfusion PET is a viable option because of its low radiation exposure (score 8).

Clinical Scenarios 142-147: Patients with Familial Hypercholesterolemia. In symptomatic patients with familial hypercholesterolemia (scenarios 142-146) without evidence of obstructive CAD by imaging, no specific outcome data are available. Nonetheless, the application of PET MPI was deemed appropriate to identify CMD as a potential substrate for angina symptoms in persistent chest pain syndrome $(2,85,102,105,145-148)$ amenable to intensified preventive and vasoactive medical care with statins or ranolazine $(2,3,121,126,127)$ to control symptoms and to potentially improve outcome, respectively.

Conversely, in asymptomatic individuals with familial hypercholesterolemia (scenario 147), the application of PET MPI was deemed rarely appropriate. In rare instances, however, despite the absence of symptoms, PET MPI may be indicated for cardiovascular risk stratification if there is a positive family history of CAD.

Scenario 142: Symptomatic individuals with familial hypercholesterolemia and negative stress-rest imaging findings but persistent chest pain syndrome (Score 7 - Appropriate) 
In symptomatic individuals with familial hypercholesterolemia and negative stress-rest SPECT imaging or echocardiographic findings, but persistent chest pain syndrome, an additional stress-rest PET MPI can be performed to assess MFR and thus the presence of CMD as a potential substrate for angina symptoms $(2,85,102,104,105)$ and cardiovascular risk stratification $(29,39,46,48,114,118,120)$. Such symptomatic patients without evidence of obstructive CAD but with CMD may benefit from ACE-I, ARB, or ranolazine to improve hyperemic flow and symptoms $(2,3,121,123,124,126,127)$. From the available outcome data for CMD, clinical decision-making process, and treatment consequences, this scenario was scored as appropriate (score 7).

Scenario 143: Symptomatic individuals with familial hypercholesterolemia and positive or negative results of an exercise ECG stress test, who have atherosclerosis and nonobstructive $\mathrm{CAD}$, but persistent chest pain (Score 8 - Appropriate)

In symptomatic individuals with familial hypercholesterolemia and positive or negative results of an exercise ECG stress test, who have atherosclerosis and nonobstructive $\mathrm{CAD}$, but persistent chest pain, stress-rest PET MPI can be performed to assess MFR and thus the presence of $\mathrm{CMD}$ as a potential substrate for angina symptoms $(2,85,102,104,105)$ and cardiovascular risk stratification $(29,39,46,48,114,118,120)$. The prevalence of CMD may be as high as $60 \%$ (102). Such symptomatic patients without obstructive CAD but with CMD may benefit from ACE-I, ARB, or ranolazine to improve hyperemic flow and symptoms $(2,3,121,123,124,126,127)$. From the available outcome data for CMD and in view of the clinical decision-making process and treatment consequences, this scenario was scored as appropriate (score 8).

Scenario 144: Symptomatic individuals with familial hypercholesterolemia and positive or negative results of an exercise ECG stress test and normal results on a coronary angiogram, but persistent chest pain (Score 7 - Appropriate)

In symptomatic individuals with familial hypercholesterolemia and positive or negative results of an exercise ECG stress test and normal coronary angiogram results, but persistent chest pain, stress-rest PET MPI can be performed to assess MFR and thus the presence of CMD as a potential substrate for angina symptoms $(2,85,102,104,105)$ and cardiovascular risk stratification $(29,39,46,48,114,119,120)$. Despite normal coronary angiogram results, CMD in this population is frequently encountered (102). Such symptomatic patients with normal coronary arteries but with CMD may benefit from ACE-I, ARB, or ranolazine to improve hyperemic flow and symptoms $(2,3,121,123,124,126,127)$. From the available outcome data for CMD, clinical decision-making process, and treatment consequences, this scenario was scored as appropriate (score 7).

Scenario 145: Symptomatic individuals with familial hypercholesterolemia and positive or negative results of an exercise ECG stress test, who have no coronary artery calcifications on noncontrast cardiac CT (CCT) (Score 7 - Appropriate)

In symptomatic individuals with familial hypercholesterolemia and positive or negative results of an exercise ECG stress test, who have no coronary artery calcifications on noncontrast CCT, stressrest PET MPI can be performed to assess obstructive CAD (albeit this is less likely with the absence of coronary artery calcifications) and to measure MFR and thus the presence of CMD as a potential substrate for angina symptoms $(2,85,102,104,105)$ and cardiovascular risk stratification $(29,39,46,48,114,118,120)$. Despite the absence of coronary artery calcifications on noncontrast CCT, CAD may be present and CMD in this population is frequently encountered (102). Such symptomatic patients without apparent CAD but with CMD may benefit from ACE-I, ARB, or ranolazine to improve hyperemic flow and symptoms $(2,3,121,123,124,126,127)$. From the available outcome data for CMD, clinical decision-making process, and treatment consequences, this scenario was scored as appropriate (score 7).

Scenario 146: Symptomatic individuals with familial hypercholesterolemia and positive or negative results of an exercise ECG stress test, who have coronary artery calcifications on noncontrast CCT (Score 8 - Appropriate)

In symptomatic individuals with familial hypercholesterolemia with positive or negative results of an exercise ECG stress test, who have coronary artery calcifications on noncontrast CCT, stressrest PET MPI can be performed to identify obstructive CAD and, if perfusion results are normal, to assess CMD as a potential substrate for angina symptoms $(2,85,102,104,105)$ and for cardiovascular risk stratification $(29,39,46,48,114,118,120)$. Cardiac perfusion PET may be given preference over stress-rest SPECT because of its low radiation exposure and added value of CMD assessment. CMD in this population is frequently encountered (102). Such symptomatic patients with coronary artery calcifications but with CMD may benefit from ACE-I, ARB, or ranolazine to improve hyperemic flow and symptoms $(2,3,121,123,124,126,127)$. From the available outcome data for CMD, clinical decision-making process, and treatment consequences, this scenario was scored as appropriate (score 8).

Scenario 147: Asymptomatic individuals with familial hypercholesterolemia and positive or negative results of an exercise ECG stress test (Score 2 - Rarely Appropriate)

In asymptomatic individuals with familial hypercholesterolemia and positive or negative results of an exercise ECG stress test, stress-rest PET MPI may be used in rare instances for cardiovascular risk stratification if a positive familial history of CAD is present. In the absence of outcome data, given the clinical-decision making process and treatment options, this scenario was scored as rarely appropriate (score 2).

Clinical Scenarios 148-150: Symptomatic Patients with CAD with Known Left Main or Multivessel Disease. In symptomatic CAD patients with known left main or multivessel disease (scenarios 148-150), the application of PET MPI affords the advantage of high diagnostic accuracy, concurrent evaluation of myocardial perfusion (radiotracer uptake) and regional MFR, and wall motion assessment at peak pharmacologic stress $(2,8,10,29,35,37,39)$. Given these advantages of stress-rest perfusion PET over other imaging modalities that may profoundly affect the diagnostic accuracy, decision-making process, and treatment options, its application was deemed appropriate.

Scenario 148: Symptomatic patients with known left main or multivessel disease as shown by ICA (Score 9 - Appropriate)

In symptomatic patients with known left main or multivessel disease as shown by ICA, stress-rest perfusion PET not only determines the relative radiotracer uptake (perfusion distribution) of the left ventricle, but it also quantifies MFR and determines LV wall motion at peak stress. Alternatively, dobutamine echocardiography is a viable option if patients have normal LV function and are suitable for this technique. Stress-rest myocardial perfusion SPECT may miss diffuse ischemia induced by left main or multivessel disease, as the relative radiotracer uptake 
may be homogeneous and ischemia-induced hypokinesis or akinesis may have resolved 40 min after stress at image acquisition of ${ }^{99 \mathrm{~m}}$ Tc SPECT. Given the advantages of stress-rest perfusion PET over other imaging modalities that may profoundly affect the diagnostic accuracy $(2,8,10,29,35,37,39)$, decision-making process, and treatment options, this scenario was scored as appropriate (score 9).

Scenario 149: Symptomatic patients with CAD in the left main segment or in all 3 vessels as shown by noninvasive CTA (Score 9 - Appropriate)

In symptomatic patients with CAD in the left main segment or in all 3 vessels as shown by noninvasive CTA $(56,155,156)$, stressrest perfusion PET can be performed, as it may be seen as the most accurate and cost-effective noninvasive imaging modality in this condition. Given the advantages of stress-rest perfusion PET over other imaging modalities (as mentioned in scenario 148) that may profoundly affect the diagnostic accuracy $(2,8,10,29,35,37,39)$, decision-making process, and treatment options, this scenario was scored as appropriate (score 9).

Scenario 150: Symptomatic patients with coronary artery calcifications in the left main segment or in all 3 vessels as shown on noncontrast CCT (Score 9 - Appropriate)

In symptomatic patients with coronary artery calcifications in the left main segment or in all 3 vessels as shown on noncontrast CCT $(56,155,156)$, stress-rest perfusion PET can be performed, as it may be seen as the most accurate and cost-effective noninvasive imaging modality in this condition. Given the advantages of stress-rest perfusion PET over other imaging modalities (as mentioned in scenario 148) that may profoundly affect the diagnostic accuracy $(2,8,10,29,35,37,39)$, decision-making process, and treatment options, this scenario was scored as appropriate (score 9).

Clinical Scenarios 151-153: Asymptomatic Patients with CAD with Known Left Main or Multivessel Disease. Conversely, in asymptomatic patients with known left main or multivessel disease (scenarios 151-153), the application of PET MPI may be justified in view of the possibility of advanced CAD-induced repetitive silent ischemia that may eventually lead to heart failure or sudden cardiac death. Given the appropriate clinical context, PET MPI may be indicated for this scenario.

Scenario 151: Asymptomatic patients with known left main or multivessel disease as shown by coronary angiography (Score 6 - May be Appropriate)

In asymptomatic patients with known left main or multivessel disease as shown by coronary angiography $(56,155,156)$, stressrest perfusion PET can be performed to identify silent macro- or microvascular CAD that could lead to heart failure or sudden cardiac death. Although the patient is asymptomatic, the risk constellation shown from coronary morphologic features with left main or multivessel disease may necessitate further risk stratification with evaluation for ischemia with stress-rest perfusion PET. Given this scenario, stress-rest perfusion PET may be most helpful for further risk stratification $(2,8,10,29,35,37,39)$ that potentially affects the decision-making process and treatment options. Thus, this scenario was scored as may be appropriate (score 6).

Scenario 152: Asymptomatic patients with pronounced coronary calcifications in the left main or in all 3 main vessels as shown by noncontrast CCT (Score 7 - Appropriate)
In asymptomatic patients with pronounced coronary calcifications in the left main or in all 3 main vessels as shown on noncontrast CCT $(56,155,156)$, stress-rest perfusion PET can be performed to identify silent macro- or microvascular CAD that could lead to heart failure or sudden cardiac death. Although the patient is asymptomatic, the risk constellation of pronounced coronary calcifications in the left main or in all 3 main vessels necessitates further risk stratification by evaluation for ischemia with stress-rest perfusion PET $(2,8,10,29,35,37,39)$. Given the outcome data, decision-making process, and treatment options, this scenario was scored as appropriate (score 7).

Scenario 153: Asymptomatic patients with mild coronary calcifications in the left main or in all 3 main vessels as shown on noncontrast CCT (Score 3 - Rarely Appropriate)

In asymptomatic patients with mild coronary calcifications in the left main or in all 3 main vessels as shown on noncontrast CCT $(56,155,156)$, stress-rest perfusion PET can be performed to assess silent macro- or microvascular CAD. Patients who are asymptomatic and have mild coronary artery calcifications do not necessarily have an increased risk for silent ischemia. Given the outcome data, low influence on the decision-making process, and treatment options, this scenario was scored as rarely appropriate (score 3).

Clinical Scenarios 154-157: Patients Who Have Undergone $C A B G$. In symptomatic patients with CAD who have undergone CABG, the application of PET MPI was deemed appropriate if exercise SPECT cannot be performed (scenario 154), or if exercise SPECT findings are equivocal (scenario 155) $(27,28)$.

Conversely, in asymptomatic patients with CAD who have undergone CABG, the application of PET MPI within $5 \mathrm{y}$ of CABG (scenario 156) was deemed as rarely appropriate (low diagnostic yield), whereas for a $\geq 5$-y period after CABG (scenario 157) PET MPI may be appropriate for the detection of silent ischemia due to the increased likelihood of occlusion of single bypass grafts $(27,28)$.

Scenario 154: Symptomatic patients after undergoing CABG: stress-rest perfusion PET if exercise SPECT cannot be performed (Score 8 -Appropriate)

In symptomatic patients who have undergone CABG, stressrest perfusion PET can be performed if exercise SPECT is not feasible. From the outcome data $(29,39,46,48,114,118,120)$, advantages of stress-rest perfusion PET that may affect the decision-making process (evaluation of each lesion for its hemodynamic consequences with regional MFR assessment) $(2,8,9,39)$, and treatment options (redo CABG or PCI), this scenario was scored as appropriate (score 8).

Scenario 155: Symptomatic patients after undergoing CABG: stress-rest perfusion PET if exercise SPECT is equivocal (Score 8 - Appropriate)

In symptomatic patients who have undergone CABG, stressrest perfusion PET can be performed if exercise SPECT is equivocal. Given the advantages of stress-rest perfusion PET, leading to the highest diagnostic accuracy $(2,8,9,39)$, this scenario was scored as appropriate (score 8).

Scenario 156: Asymptomatic patients within 5 y of undergoing CABG (Score 2 - Rarely Appropriate)

In asymptomatic patients within $5 \mathrm{y}$ of undergoing $\mathrm{CABG}$, the diagnostic yield of stress-rest perfusion PET would be low or 
nonexistent $(27,28)$. Thus, this scenario was scored as rarely appropriate (score 2 ).

Scenario 157: Asymptomatic patients $\geq 5$ y after undergoing CABG (Score 6 - May be Appropriate)

In asymptomatic patients $\geq 5 \mathrm{y}$ after undergoing CABG, stressrest perfusion PET can be performed, as there is a certain probability of silent ischemia because of the potential occlusion of single bypass grafts. Given the severity of CAD and the numbers of left internal mammary artery and saphenous vein graft bypasses $(27,28)$, this scenario was scored as may be appropriate (score 6).

Clinical Scenarios 158-161: Patients Who Have Undergone PCI in Multivessel Disease. In symptomatic patients with CAD who have undergone PCI in multivessel disease, the application of PET MPI was deemed appropriate if exercise SPECT cannot be performed (scenario 158), or if exercise SPECT findings are equivocal (scenario 159) $(27,28)$.

Conversely, in asymptomatic patients with CAD who have undergone PCI in multivessel disease, the application of PET MPI within 2 y of PCI (scenario 160) was deemed rarely appropriate (low diagnostic yield), whereas at $\geq 2$ y after PCI (scenario 161), PET MPI may be appropriate for the detection of silent ischemia because of the increased likelihood of silent ischemia from a novel stenosis in the arterial vessels $(27,28)$.

Scenario 158: Symptomatic patients with known multivessel disease who have undergone PCI in whom exercise SPECT cannot be performed (Score 8 - Appropriate)

In symptomatic patients with known multivessel disease who have undergone PCI, stress-rest perfusion PET can be performed if exercise SPECT is not feasible. From the outcome data $(29,39,46,48,114,118,120)$, the advantages of stress-rest perfusion PET that may affect the decision-making process (evaluation of each lesion for its hemodynamic consequences, with regional MFR assessment) (2,8,9,39), and the treatment options (redo CABG or PCI), this scenario was scored as appropriate (score 8).

Scenario 159: Symptomatic patients with known multivessel disease who have undergone PCI and who have equivocal exercise SPECT results (Score 9 - Appropriate)

In symptomatic patients with known multivessel disease who have undergone PCI, stress-rest perfusion PET can be performed if exercise SPECT is equivocal. Given the advantages of stressrest perfusion PET, leading to the highest diagnostic accuracy $(2,8,9,39)$, this scenario was scored as appropriate (score 9).

Scenario 160: Asymptomatic patients within 2 y of undergoing PCI (Score 2 - Rarely Appropriate)

In asymptomatic patients within $2 \mathrm{y}$ of undergoing PCI, the diagnostic yield of stress-rest perfusion PET would be low or nonexistent $(27,28)$. Thus, this scenario was scored as rarely appropriate (score 2 ).

Scenario 161: Asymptomatic patients $\geq 2$ y after undergoing PCI (Score 6 - May be Appropriate)

In asymptomatic patients at $\geq 2$ y after undergoing PCI, stressrest perfusion PET can be performed, as there is a certain probability of silent ischemia because of novel stenosis in the arterial vessels. Given the complexity of CAD and number of PCIs that include stents $(27,28)$, this scenario was scored as may be appropriate (score 6).

Clinical Scenarios 162-163: Use of Rest or Stress-Rest PET Perfusion in Patients in Conjunction with ${ }^{18}$ F-FDG PET to Assess Cardiac Sarcoidosis. In patients with suspected cardiac sarcoidosis (scenario 162), resting PET MPI is an integral part of the ${ }^{18} \mathrm{~F}-\mathrm{FDG}$ PET protocol in the detection and characterization of cardiac sarcoidosis and thus this scenario was deemed appropriate $(86,157,158)$.

In patients with suspected cardiac sarcoidosis with angina or chest pain symptoms or dyspnea that raises the suspicion for concomitant CAD (scenario 163), adding stress MPI PET to rest MPI and ${ }^{18}$ F-FDG PET was deemed appropriate $(86,157-159)$.

Scenario 162: Patients with suspected cardiac sarcoidosis: rest perfusion PET as an integral part of the ${ }^{18} \mathrm{~F}$-FDG PET protocol in the detection and characterization of cardiac sarcoidosis (Score 8 - Appropriate)

In patients with suspected cardiac sarcoidosis, resting perfusion PET is an integral part of the ${ }^{18} \mathrm{~F}$-FDG PET protocol in the detection and characterization of cardiac sarcoidosis (86,157-159). The presence of a relative perfusion abnormality may signify old cardiac sarcoid activity as scarring or severe fibrosis, or it may reflect severe edema in the acute setting. In addition, a so-called mismatch between reduced perfusion and abnormal ${ }^{18} \mathrm{~F}$-FDG uptake with a typical location (basal segments of the LV wall) is highly diagnostic for active inflammatory cardiac sarcoidosis. Overall, adding rest MPI to ${ }^{18} \mathrm{~F}$-FDG PET increases the diagnostic yield, specificity, and reader confidence in the detection and characterization of cardiac sarcoidosis $(86,157,158)$. Thus, this scenario was scored as appropriate (score 8 ).

Scenario 163: Patients with suspected cardiac sarcoidosis and concurrent chest pain symptoms or dyspnea who are undergoing rest perfusion and the ${ }^{18} \mathrm{~F}-\mathrm{FDG}$ PET protocol: addition of stress perfusion PET (Score 8 - Appropriate)

In patients with suspected cardiac sarcoidosis who are undergoing rest perfusion and the ${ }^{18} \mathrm{~F}$-FDG PET protocol, stress perfusion can be added in those with angina, chest pain symptoms, or dyspnea that raises the suspicion for concomitant CAD $(86,157-159)$. Exercise or CAD-induced ischemia may lead to regional ${ }^{18} \mathrm{~F}$-FDG-uptake and thus false-positive findings in sarcoid detection. False-positive findings on ${ }^{18} \mathrm{~F}-\mathrm{FDG}$ PET images can be avoided by acquiring stress (-rest) perfusion images the day after ${ }^{18}$ F-FDG PET image acquisition to prevent interference with the ${ }^{18}$ F-FDG PET examination $(86,158)$. From the clinical decision-making process and treatment options, this scenario was scored as appropriate (score 8).

Clinical Scenario 164: Use of Stress-Rest Perfusion in Patients in Conjunction with ${ }^{18}$ F-FDG PET to Assess Myocardial Viability.

Scenario 164: Patients with assessment of myocardial viability in ischemic cardiomyopathy: rest or stress perfusion PET as an integral part of the ${ }^{18} \mathrm{~F}$-FDG PET protocol in the detection and characterization of myocardial viability (Score 9 Appropriate)

In patients with assessment of myocardial viability in ischemic cardiomyopathy, rest or stress perfusion PET is an integral part of the ${ }^{18} \mathrm{~F}$-FDG PET protocol in the detection and characterization of myocardial viability. Concurrent resting perfusion assessment is an integral part of the diagnostic process. ${ }^{18} \mathrm{~F}-\mathrm{FDG}$ uptake may 
exceedingly increase in regions with ischemically compromised viable or hibernation-stunned myocardium. Within the normalization process of the ${ }^{18} \mathrm{~F}-\mathrm{FDG}$ signal, the excessive uptake of ${ }^{18} \mathrm{~F}$ FDG may be upscaled (100\%) and the remaining viable regions therefore may be downscaled pretending regions with reduced (albeit normal) viability. In some instances, the ${ }^{18} \mathrm{~F}-\mathrm{FDG}$ uptake may only occur in regions with viable but ischemically compromised myocardium. Resting perfusion in these regions with low or no ${ }^{18}$ F-FDG uptake will be normal and thus indicate normal viability $(26,27)$. Adding stress perfusion assessment with PET may add diagnostic value, as the hemodynamic effects of each CAD lesion in multivessel disease can be evaluated by the concurrent assessment of MFR, which may help in the decision-making process for complete or incomplete revascularization (9). Thus, this scenario was scored as appropriate (score 9).

Clinical Scenario 165: Patients Who Are Apparent Nonresponders to Pharmacologic Vasodilation with a Need to Change to Dobutamine Stress Testing.

Scenario 165: Symptomatic patients with an intermediate probability of CAD who are undergoing functional testing with myocardial perfusion SPECT or PET during pharmacologic stimulation and rest: repeat imaging test with PET but with dobutamine stimulation if there is no hemodynamic response (change in arterial blood pressure or no heart rate increase) during pharmacologic vasodilator stress (Score 3 Rarely Appropriate)

This issue is controversial, but given the results of the Rampart trial (160) without a correlation among hyperemic coronary flow increases and hemodynamic response during pharmacologic vasodilator stress, this scenario was deemed rarely appropriate (score 3).

Clinical Scenario 166: Patients with Transplant Vasculopathy.

Scenario 166: Symptomatic patients or patients with suspected cardiac graft rejection (Score 8 - Appropriate)

In symptomatic patients or in those with suspected cardiac graft rejection, PET MPI is unique, as it provides concurrent information about the presence of epicardial obstructive lesions and potential reductions in MFR, reflecting diffuse transplant vasculopathy and CMD $(72,73,84,161)$. In addition, the identification of the severity of CMD may provide critical information about subclinical graft rejection (72). Given these advantages, PET MPI was deemed appropriate (score 8).

Clinical Scenario 167: Patients with Vasculitis and Arteritis (Kawasaki and Takayasu Disease).

Scenario 167: Symptomatic patients with suspected vasculitis and arteritis (Score 8 - Appropriate)

In symptomatic patients with suspected vasculitis and arteritis, PET MPI is unique, as it provides concurrent information about the presence of epicardial obstructive lesions and potential reductions in MFR, reflecting diffuse CMD. These patients have a poor outcome if the coronary vessels are affected, leading to ischemia and CMD $(152,162)$. Although no specific outcome data are available related to cardiac perfusion PET, given the advantage of PET MPI to concurrently assess CMD, this clinical scenario was deemed appropriate (score 8).

\section{Summary of Recommendations}

These compiled clinical scenarios outline the appropriate use of PET perfusion and flow quantification, and the reasons behind it, in specific populations. PET perfusion imaging has a high diagnostic accuracy, sensitivity, and specificity not attained by other imaging modalities when used in symptomatic individuals with advanced obesity and in women with large dense breasts. In addition, the concurrent assessment of microvascular (dys)function, in particular in postmenopausal women, adds important diagnostic information and opens up treatment options when the results of stress-rest PET MPI are normal (representing nonobstructive or no CAD) in the same assessment. The low radiation exposure of PET perfusion imaging also renders it more suitable in these specific populations, as well as in younger ages and in individuals with congenital heart disease (if exercise stress testing is not needed). In symptomatic individuals with familial hypercholesterolemia, PET perfusion imaging is commonly indicated to rule out hemodynamically significant obstructive CAD and, at the same time, to evaluate for the presence of CMD as a potential cause for chest pain syndrome. Conversely, PET flow quantification is not indicated for cardiovascular risk stratification in asymptomatic individuals in this specific population. The reasoning for the preferential use of PET perfusion and flow quantification in patients with known left main or multivessel disease has been outlined in detail: high diagnostic accuracy, regional flow quantification, and peak stress wall motion measurements in CAD assessment and characterization. PET perfusion assessment at rest is an integral part of the diagnostic process in the assessment of myocardial viability in patients with ischemic cardiomyopathy and in those undergoing ${ }^{18} \mathrm{~F}$-FDG PET evaluation for the identification of active inflammatory cardiac sarcoidosis. In symptomatic patients after cardiac transplantation, PET perfusion and flow quantification is the preferred noninvasive imaging modality, as it detects not only flow-limiting obstructive CAD lesions (stress-related regional perfusion deficit), but also diffuse transplant vasculopathy (reduction in MFR). For similar reasons, the role of PET perfusion and flow quantification was seen as appropriate in symptomatic patients with Kawasaki or Takayasu disease.

\section{SECTION 9: PRIOR TESTING OR PROCEDURES}

\section{Introduction and Background}

Considerations of the appropriateness of PET MPI include symptom status, type and results of prior testing, timing since prior testing, and prior revascularization of the patient. Appropriate testing guidelines for SPECT MPI generally apply to PET MPI, but PET MPI has additional appropriateness considerations. ECGgated PET MPI can monitor serial quantitative changes in myocardial perfusion, cardiac size and function associated with lifestyle, and medical and revascularization therapies. These factors influence the appropriate use of PET MPI for the diagnosis, prognosis, and monitoring of treatment response and can guide new or more intensive lifestyle, medical, and revascularization therapies in patients with known or suspected ischemic heart disease.

\section{Clinical Scenarios and AUC Scores}

Table 9 presents the following clinical scenarios and final AUC scores for the use of PET MPI in prior testing or procedures.

Clinical Scenarios 168-173: Abnormal Results of a Prior Exercise ECG Test.

Scenario 168: Abnormal results of a prior exercise ECG test $<$ $90 \mathrm{~d}$ ago with no intervening revascularization or sequential testing (Score 1 - Rarely Appropriate) 
TABLE 9

Clinical Scenarios for the use of PET MPI After Prior Testing or Procedures

\begin{tabular}{|c|c|c|c|}
\hline Scenario no. & Description & Appropriateness & Score \\
\hline \multicolumn{4}{|c|}{ Abnormal results of a prior exercise ECG test } \\
\hline 168 & $\begin{array}{l}\text { Abnormal results of a prior exercise ECG test }<90 \mathrm{~d} \text { ago with no intervening } \\
\text { revascularization and sequential testing }\end{array}$ & Rarely Appropriate & 1 \\
\hline 169 & Abnormal results of a prior exercise ECG test & May be Appropriate & 5 \\
\hline 170 & Abnormal results of a prior exercise ECG test with a low-risk DTS $(>10)$ & May be Appropriate & 6 \\
\hline 171 & $\begin{array}{l}\text { Abnormal results of a prior exercise ECG test with an intermediate-risk DTS } \\
(-10 \text { to }+4)\end{array}$ & Appropriate & 8 \\
\hline 172 & Abnormal results of a prior exercise ECG test with a high-risk DTS $(<-10)$ & Appropriate & 8 \\
\hline 173 & ETT achieving $\geq 10$ METs with no ischemic ST depression & May be Appropriate & 4 \\
\hline \multicolumn{4}{|c|}{ Abnormal results of a prior stress imaging study } \\
\hline 174 & Abnormal results of a prior stress imaging study & Rarely Appropriate & 3 \\
\hline 175 & Equivocal or discordant results of a prior stress test with or without imaging & Appropriate & 8 \\
\hline 176 & CAD of uncertain clinical significance on prior CCTA or ICA & Appropriate & 8 \\
\hline 177 & $\begin{array}{l}\text { Abnormal prior CCT calcium results (Agatston score }<100 \text { ) in asymptomatic } \\
\text { patients }\end{array}$ & Rarely Appropriate & 2 \\
\hline 178 & $\begin{array}{l}\text { Abnormal prior CCT calcium results (Agatston score 100-400) in asymptomatic } \\
\text { patients }\end{array}$ & May be Appropriate & 5 \\
\hline 179 & $\begin{array}{l}\text { Abnormal prior CCT calcium results (Agatston score }>1,000 \text { ) in asymptomatic } \\
\text { patients }\end{array}$ & Appropriate & 8 \\
\hline \multicolumn{4}{|c|}{ Follow-up testing (>90 d): asymptomatic patients } \\
\hline \multicolumn{4}{|c|}{ After abnormal results of a prior exercise ECG test } \\
\hline 180 & Last test $<2$ y ago & Rarely Appropriate & 1 \\
\hline 181 & Last test $\geq 2$ y ago & Rarely Appropriate & 1 \\
\hline \multicolumn{4}{|c|}{ After abnormal results of a prior stress imaging study (non-PET) } \\
\hline 182 & Last test $<2$ y ago & Rarely Appropriate & 2 \\
\hline 183 & Last test $\geq 2$ y ago & Rarely Appropriate & 1 \\
\hline \multicolumn{4}{|c|}{ Follow-up testing ( $>90 \mathrm{~d}$ ): patients with stable symptoms } \\
\hline \multicolumn{4}{|c|}{ After abnormal results of a cardiovascular imaging test } \\
\hline 184 & Last test $<2$ y ago & Rarely Appropriate & 1 \\
\hline 185 & Last test $\geq 2$ y ago & May be Appropriate & 4 \\
\hline \multicolumn{4}{|c|}{ After abnormal results of a prior stress imaging study (PET) } \\
\hline 186 & Abnormal results of prior PET MPI 3-6 mo ago & Rarely Appropriate & 1 \\
\hline 187 & Abnormal results of prior PET MPI $>6$ mo ago & Rarely Appropriate & 2 \\
\hline \multicolumn{4}{|c|}{ After obstructive CAD as shown on prior coronary angiography (invasive or noninvasive) } \\
\hline 188 & Last test $<2$ y ago & May be Appropriate & 6 \\
\hline 189 & Last test $\geq 2$ y ago & May be Appropriate & 6 \\
\hline \multicolumn{4}{|c|}{ After normal results of a prior exercise ECG test } \\
\hline 190 & Low global CAD risk $(<7.5 \%)$ & Rarely Appropriate & 1 \\
\hline 191 & Intermediate CAD risk (7.5\%-20\%) when tested $<2$ y ago & Rarely Appropriate & 2 \\
\hline 192 & Intermediate CAD risk $(7.5 \%-20 \%)$ when tested $\geq 2$ y ago & Rarely Appropriate & 3 \\
\hline 193 & High global CAD risk ( $>20 \%$ ) when tested $<2$ y ago & May be Appropriate & 4 \\
\hline 194 & High global CAD risk $(>20 \%$ ) when tested $\geq 2$ y ago & May be Appropriate & 5 \\
\hline
\end{tabular}

PET MPI $<90 \mathrm{~d}$ after abnormal results of a prior exercise ECG test without intervening revascularization is considered rarely appropriate (score 1). A goal-directed lifestyle and medical therapeutic intervention with or without revascularization appears to be appropriate without additional perfusion imaging (163). Serial MPI can identify a reduction in stress perfusion defects and ischemia consistent with a therapeutic response shown by using SPECT MPI or PET MPI (164-166). Although studies have shown incremental risk assessment with quantitative assessment of global myocardial perfusion reserve (MPR) of PET MPI $(46,167)$, reclassification of patients is unlikely to affect clinical management in this group with PET MPI $<90$ 
d after an abnormal prior exercise ECG test without intervening revascularization. If the abnormality on the prior ECG is nondiagnostic of ischemia, for example, with nonspecific t-wave abnormalities or a conduction abnormality such as LBBB, the test would be considered indeterminate for ischemia, and PET MPI would be categorized as appropriate. PET MPI is considered appropriate after equivocal or nondiagnostic categorization of the abnormality on the stress SPECT MPI or prior exercise ECG stress test.

Scenario 169: Abnormal results of a prior exercise ECG test (Score 5 - May be Appropriate)

Scenario 170: Abnormal results of a prior exercise ECG test with a low-risk Duke treadmill score (DTS) ( $\geq 5)$ (Score $6-$ May be Appropriate)

Scenario 171: Abnormal results of a prior exercise ECG test with an intermediate-risk DTS $(-10$ to +4$)$ (Score $8-$ Appropriate)

Scenario 172: Abnormal results of a prior exercise ECG test with a high-risk DTS $(>+4)$ (Score 8 - Appropriate)

PET MPI after abnormal results of a prior exercise ECG test may be appropriate (score 5). The degree of abnormality of the DTS (168) influences the likelihood that SPECT MPI or PET MPI can provide incremental risk assessment (169). DTS is calculated as follows: (number of minutes of Bruce protocol exercise time) $(5 \times \mathrm{mm}$ of ST segment deviation from baseline $)-(4 \times$ exercise treadmill test [ETT] angina index [0 for none, 1 for some, 2 for limiting angina during ETT]). With normal and severely abnormal MPI results, patients with intermediate-risk DTS (fatal and nonfatal MI risk $2.5 \%)$ are reclassified to low-risk $(<0.1 \%-0.4 \%)$ and high-risk ( $8.9 \%$ per year) categories, respectively, and the relative risk of these hard coronary events within intermediate-risk DTS is amplified by more than 22-fold $(169,170)$. The high diagnostic and prognostic accuracy of PET MPI $(29,171,172)$ can be expected to reliably reclassify the risk assignment in this intermediate-risk DTS group. SPECT MPI and PET MPI are appropriate (score 8 ) in the intermediate-risk (DTS: -10 to +4 ) and highrisk (DTS: $<-10$ ) groups, and may be appropriate (score 6) in the low-risk group (DTS: $>4$ ), similar to that for SPECT MPI $(29,171,172)$.

Scenario 173: ETT of $\geq 10$ metabolic equivalents (METs) with no ischemic ST depression (Score 4 - May be Appropriate)

Bourque et al. reported that patients at intermediate risk for CAD or known CAD who achieve $\geq 10$ METs have a very low prevalence of $\geq 10 \%$ LV ischemia and very low rates of cardiac mortality, nonfatal MI, and late revascularization, irrespective of the heart rate achieved (173). Cardiac events did not correlate with abnormalities on the index MPI study. This study suggested that patients who attain $\geq 10$ METs during exercise stress have an excellent prognosis over an intermediate follow-up period, regardless of the peak exercise heart rate achieved. The added value of radionuclide MPI with standard exercise ECG testing in this population is questionable. However, radionuclide MPI demonstrated a 26-fold incremental risk assessment for hard coronary events in similar low-risk DTS patients in a study by Hachamovitch et al. (169). Thus, PET MPI may be appropriate (score 4) in this low- risk group without ST depression at high aerobic capacity of $>10$ METs during ETT.

Clinical Scenarios 174-179: Abnormal or Uncertain Results of a Prior Stress Imaging Study.

Scenario 174: Abnormal results of a prior stress imaging study (Score 3 - Rarely Appropriate)

PET MPI after abnormal results of a prior stress imaging study is considered rarely appropriate (score 3 ). The rationale for this assessment was stated in scenario 168, as a goal-directed lifestyle and medical therapeutic intervention with or without revascularization appears to be appropriate without additional perfusion imaging (163).

Scenario 175: Equivocal or discordant results of a prior stress test with or without imaging (Score 8 - Appropriate)

PET MPI after equivocal or discordant results of a prior stress test with or without imaging is appropriate (score 8). PET provides improved image quality and diagnostic accuracy associated with attenuation correction, coincident detection, and higher sensitivity detectors, which provide higher count rate statistics to help resolve equivocal or discordant results of prior imaging or nonimaging stress tests $(171,172)$.

Scenario 176: CAD of uncertain clinical significance on prior CCTA or ICA (Score 8 - Appropriate)

PET MPI to assess CAD of uncertain clinical significance on prior CCTA or ICA is appropriate (score 8). Similar to SPECT MPI, PET MPI provides favorable clinical value in the diagnosis, prognosis, and cost-effective management of known or suspected ischemic heart disease.

Scenario 177: Abnormal prior CCT calcium results (Agatston score < 100) (Score 2 - Rarely Appropriate)

PET MPI after prior abnormal low CCT calcium results (Agatston score $<100$ ) is rarely appropriate (score 2). The likelihood of morbid coronary events over $10 \mathrm{y}$ in this group of asymptomatic patients with multiple coronary risk factors is very low $(<10 \%)$ and is affected by sex and Framingham risk profile.

Scenario 178: Abnormal prior CCT calcium results (Agatston score 100-400) (Score 5 - May be Appropriate)

PET MPI after prior abnormal intermediate CCT calcium results (Agatston score 100-400) may be appropriate (score 5). The likelihood of morbid coronary events over $10 \mathrm{y}$ in this group of asymptomatic patients with multiple coronary risk factors is significant $(10 \%-20 \%)$.

Scenario 179: Abnormal prior CCT calcium results (Agatston score 400-1,000 or >1,000) (Score 8 - Appropriate)

PET MPI after prior abnormal high CCT calcium results (Agatston score 400-1,000 or $>1,000$ ) is appropriate (score 8). The likelihood of morbid coronary events over $10 \mathrm{y}$ in this group of asymptomatic patients with multiple coronary risk factors is substantial $(>20 \%)(174,175)$.

ACC/AHA guidelines acknowledge that measurement of CAC can be useful for cardiovascular risk assessment in asymptomatic adults at intermediate or higher risk (10-y risk) (176). PET MPI may be appropriate to provide further incremental risk 
assessment of CAD, depending on the level of coronary calcium observed.

Clinical Scenarios 180-183: Follow-up Testing (>90 Days): Asymptomatic Patients. The following scenarios address abnormal results of a prior exercise ECG test.

Scenario 180: Last test $<2$ y ago (Score 1 - Rarely Appropriate)

Scenario 181: Last test $\geq 2$ y ago (Score 1 - Rarely Appropriate)

The following scenarios address abnormal results of a prior stress imaging study (non-PET).

Scenario 182: Last test $<2$ y ago (Score 2 - Rarely Appropriate)

Scenario 183: Last test $\geq 2$ y ago (Score 1 - Rarely Appropriate)

PET MPI is rarely appropriate in asymptomatic patients with abnormal results of a prior exercise ECG test performed $<2$ y or $\geq 2 \mathrm{y}$ ago. By this time, if indicated, guideline-directed lifestyle and medical therapy would be expected to have been implemented (163). Additional testing is unlikely to change the diagnosis or therapeutic management. However, it is recognized that in certain circumstances of delayed referral to a PET center, PET MPI may be appropriate (score 4).

Clinical Scenarios 184-194: Follow-up Testing (>90 Days): Patients with Stable Symptoms or Asymptomatic Patients. The following scenarios address abnormal results of a prior cardiovascular imaging test in patients with stable symptoms.

Scenario 184: Last test $<2$ y ago (Score 1 - Rarely Appropriate)

PET MPI is rarely appropriate (score 1) in patients with stable symptoms lasting $>90 \mathrm{~d}$ and $<2 \mathrm{y}$. By this time, if indicated, guideline-directed lifestyle and medical therapy would be expected to have been implemented (163). Additional testing is unlikely to change the diagnosis or therapeutic management. However, it is recognized that in certain circumstances of delayed referral to a PET center, PET MPI may be appropriate (score 4).

Scenario 185: Last test $\geq 2$ y ago (Score 4 - May be Appropriate)

PET MPI may be appropriate (score 4) in patients with abnormal results of a prior non-PET imaging study performed $\geq 2 \mathrm{y}$ ago. By this time, if indicated, guideline-directed lifestyle and medical therapy would be expected to have been implemented (163), but disease progression may have occurred. Additional testing in these patients with stable symptoms long after initial evaluation might change the diagnosis or therapeutic management.

The following scenarios address abnormal results of a prior stress PET in asymptomatic patients without clinical change.

Scenario 186: Abnormal results of PET MPI 3-6 mo ago (Score 1 - Rarely Appropriate)

Scenario 187: Abnormal results of PET MPI $>6$ mo ago (Score 2 - Rarely Appropriate)

PET MPI is rarely appropriate in asymptomatic patients without clinical change with abnormal results of stress PET MPI 3-6 or $>6$ mo ago. By this time, if indicated, guideline-directed lifestyle and medical therapy would be expected to have been implemented. Additional testing is unlikely to change the diagnosis or therapeutic management.

The following scenarios address obstructive CAD on prior coronary angiography (invasive or noninvasive) in asymptomatic patients without clinical change.
Scenario 188: Last test $<2$ y ago (Score 6 - May be Appropriate)

Scenario 189: Last test $\geq 2$ y ago (Score 6 - May be Appropriate)

PET MPI may be appropriate (score 6) in patients with obstructive CAD on prior coronary angiography (invasive or noninvasive) performed $<2$ y ago and $\geq 2$ y ago, depending on clinical circumstances.

The Economics of Noninvasive Diagnosis study and the PROMISE trial demonstrated the ability of functional imaging with MPI to enhance the diagnostic and prognostic accuracy and the costeffectiveness of CAD management (177-180). Classic studies on the prognostic value of MPI compared with that of ICA in men (181) and women $(108,182,183)$ demonstrate similar or higher prognostic assessment of MPI compared with that of angiographic stenoses. In the nuclear cardiology substudy of the COURAGE trial, scintigraphic reduction of ischemia associated with guideline-directed medical therapy (GDMT) with or without revascularization was associated with improved outcomes (166). Global ischemic burden can be optimally defined in patients with extensive or multivessel CAD by using dynamic flow analysis, which permits calculation of LV regional and global MFR with PET MPI $(29,46,118,167)$. Women tend to have greater risk of morbid coronary events without the degree of obstructive CAD noted in men, which can be attributable to impaired MPR $(108,183)$. Patients with stable ischemic heart disease on GDMT within 2 y of diagnosis of obstructive coronary disease by angiography would be expected to have a reduced risk of major adverse cardiac events. However, in high-risk patients with multivessel CAD, diabetes, chronic kidney disease, or statin intolerance for which more intensive GDMT may be considered, such as proprotein convertase subtilisin/kexin type 9 inhibitors, follow-up PET MPI may be appropriate to consider. New symptoms or ECG changes may prompt consideration of PET MPI in this group of patients with obstructive angiographic CAD.

The following scenarios address normal results of a prior exercise ECG test in patients with stable symptoms.

Scenario 190: Low global CAD risk $(<7.5 \%)$ (Score $1-$ Rarely Appropriate)

PET MPI is rarely appropriate (score 1) in patients with normal results of a prior exercise ECG test with stable symptoms and a low global CAD event risk of $<7.5 \%$ per decade. In these patients, further testing is highly unlikely to identify CAD that would require treatment.

Scenario 191: Intermediate CAD risk (7.5\%-20\%) when tested $<2$ y ago (Score 2 - Rarely Appropriate)

Scenario 192: Intermediate CAD risk (7.5\%-20\%) when tested $\geq 2$ y ago (Score 3 - Rarely Appropriate)

PET MPI is rarely appropriate (score 2) in patients with normal results of a prior exercise ECG test with stable symptoms for $<2 \mathrm{y}$ or an intermediate CAD event risk of $7.5 \%-20 \%$ per decade. In these patients, further testing is unlikely to identify CAD that would require treatment. PET MPI continues to be considered rarely appropriate (score 3 ) $\geq 2 \mathrm{y}$ after exercise ECG testing in these patients.

Scenario 193: High global CAD risk $(\geq 20 \%)$ when tested $<2$ y ago (Score 4 - May be Appropriate)

PET MPI may be appropriate (score 4) in patients with normal results of a prior exercise ECG test with stable symptoms for $<2$ y and with a high CAD event risk of $\geq 20 \%$ per decade. The global 
event risk of these patients indicates the need for GDMT (176), and PET MPI may identify angiographic CAD that would benefit from revascularization.

Scenario 194: High global CAD risk $(\geq 20 \%)$ when tested $\geq 2$ y ago (Score 5 - May be Appropriate)

PET MPI may be appropriate (score 5) in patients with normal results of a prior exercise ECG test with stable symptoms for $\geq 2 \mathrm{y}$ and with a high CAD event risk of $\geq 20 \%$ per decade. The global event risk of these patients indicates the need for GDMT (176), and PET MPI may identify angiographic CAD that would benefit from revascularization.

\section{Summary of Recommendations}

The clinical value of PET MPI for patients with prior testing or revascularization is critically dependent on the likelihood that the highly accurate assessment of myocardial ischemic jeopardy will result in improved understanding and tailoring of lifestyle and medical and interventional therapies and will improve clinical outcomes. Patients most likely to benefit from PET MPI are those with new signs or symptoms of ischemic disease, unexplained changes in the ECG, high residual risk, or incomplete revascularization (sometimes after prior testing by treadmill ECG); who have been assessed by calcium scoring, coronary angiography, or global coronary risk factor outcome; or who have had prior revascularization. Testing patients who are unlikely to require changes in management, and routine periodic testing of stable patients with established CAD identified by prior testing or intervention, cannot be justified and is harmful to the appropriate allocation of health-care resources. When appropriate, PET MPI may be considered an optimal test to evaluate patients with prior testing or interventions because of its quantitative nature, capacity to measure quantitative flow, high-quality imaging, ultra-low-dose radiation exposure, and high effectiveness in the diagnosis, prognosis, and assessment of therapeutic response and residual ischemic risk.

\section{SECTION 10: PREOPERATIVE EVALUATION FOR NONCARDIAC SURGERY}

\section{Introduction}

Noncardiac surgery is commonly performed with a global volume of over 200 million cases per year (184). Cardiovascular complications are also common, estimated at 10 million per year, and are associated with an increased surgical and postoperative risk, including increased short-term mortality (185). Perioperative myocardial injury (186), diagnosed by elevated high-sensitivity troponin (Tn) T levels, occurs in 16\%-20\% of noncardiac surgical patients (186) and is associated with increased 30-d mortality.

\section{Background}

The role of preoperative diagnostic testing with stress MPI has been previously well evaluated (187-197). Significant reversible perfusion defects are consistent with ischemia and identify patients at increased risk of perioperative cardiac events, including noncardiac death and MI, with high sensitivity but limited specificity. A normal result on a stress myocardial perfusion study has a high negative predictive value. A fixed perfusion defect indicates myocardial scarring and predicts long-term cardiac events rather than perioperative cardiac events.

An updated approach to the use of noninvasive testing for perioperative cardiac evaluation for $\mathrm{CAD}$ has recently been defined in the 2014 ACC/AHA Guideline on Perioperative Cardiovascular Evaluation and Management of Patients Undergoing Noncardiac Surgery (60) and in the 2013 Multimodality Appropriate Use Criteria for the Detection and Risk Assessment of Stable Ischemic Heart Disease (28). The need for noninvasive testing in patients who do not require emergency noncardiac surgery and who are without active cardiac conditions can be determined by using a stepwise approach on the basis of risk of surgery, functional capacity, and the presence of clinical risk factors. A low-risk procedure is associated with a $<1 \%$ risk and an elevated risk procedure with a $\geq 1 \%$ risk of death or MI. Functional capacity can be assessed from activities of daily living and is considered poor in patients limited to $<4$ METs during daily activities. Clinical risk factors include history of ischemic heart disease, history of compensative or prior heart failure, history of cerebrovascular disease, diabetes mellitus, and renal insufficiency (creatinine level $>2.0$ ).

\section{Clinical Scenarios and AUC Scores}

Table 10 presents the following clinical scenarios and final AUC scores for the use of PET MPI in the preoperative evaluation for noncardiac surgery.

Clinical Scenarios 195-197: Preoperative Evaluation of Patients for Noncardiac Surgery. The following scenarios address the preoperative evaluation of patients for noncardiac surgery.

Scenario 195: Patients with moderate to good functional capacity ( $\geq 4$ METs) or no clinical risk factors (history of ischemic heart disease, history of compensated or prior heart failure, history of cerebral vascular disease, diabetes mellitus, renal insufficiency) (Score 1 - Rarely Appropriate)

Patients with moderate to good functional capacity or no clinical risk factors are at low risk of cardiac complications (60) and PET MPI would be rarely appropriate.

Scenario 196: Asymptomatic patients $<1$ y after normal functional stress testing results (Score 1 - Rarely Appropriate)

Patients seen $1 \mathrm{y}$ after normal results of a functional stress test and who remain asymptomatic are unlikely to have abnormal results of a functional stress test on repeat evaluation. These patients are at low risk and PET MPI is rarely appropriate.

Scenario 197: Asymptomatic patients $<2$ y after normal coronary morphology results by either CT or invasive angiogram (Score 1 - Rarely Appropriate)

Patients seen $<2$ y after invasive or noninvasive angiography that shows normal coronary morphology results and who remain asymptomatic are unlikely to develop significant coronary stenosis. These patients are at low risk and PET MPI is rarely appropriate.

Clinical Scenarios 198-206: Patients with Poor (<4 METs) or Unknown Functional Capacity. The following scenarios address patients with poor ( $<4$ METs) or unknown functional capacity.

Scenario 198: Low-risk surgery and no clinical risk factors (Score 1 - Rarely Appropriate)

Scenario 199: Low-risk surgery and $\geq 1$ clinical risk factor (Score 2 - Rarely Appropriate)

For patients undergoing low-risk surgery, complications are low, despite poor or unknown functional capacity and with or 
TABLE 10

Clinical Scenarios for the Use of PET MPI in Preoperative Evaluation for Noncardiac Surgery

\begin{tabular}{|c|c|c|c|}
\hline Scenario no. & Description & Appropriateness & Score \\
\hline \multicolumn{4}{|c|}{ Preoperative evaluation of patients for noncardiac surgery } \\
\hline 195 & $\begin{array}{l}\text { Patients with moderate to good functional capacity ( } \geq 4 \text { METs) or no clinical risk } \\
\text { factors (history of ischemic heart disease, history of compensated or prior heart } \\
\text { failure, history of cerebral vascular disease, diabetes mellitus, renal insufficiency) }\end{array}$ & Rarely Appropriate & 1 \\
\hline 196 & Asymptomatic patients $<1$ y after normal functional stress testing results & Rarely Appropriate & 1 \\
\hline 197 & $\begin{array}{l}\text { Asymptomatic patients }<2 \text { y after normal coronary morphology results by } \\
\text { either CT or invasive angiogram }\end{array}$ & Rarely Appropriate & 1 \\
\hline \multicolumn{4}{|c|}{ Patients with poor ( $<4$ METs) or unknown functional capacity } \\
\hline 198 & Low-risk surgery and no clinical risk factors & Rarely Appropriate & 1 \\
\hline 199 & Low-risk surgery and $\geq 1$ clinical risk factor & Rarely Appropriate & 2 \\
\hline 200 & Intermediate-risk surgery and no clinical risk factors & May be Appropriate & 4 \\
\hline 201 & Intermediate-risk surgery and $\geq 1$ clinical risk factor & May be Appropriate & 5 \\
\hline 202 & Vascular surgery and no clinical risk factors & Appropriate & 7 \\
\hline 203 & Vascular surgery and $\geq 1$ clinical risk factor & Appropriate & 8 \\
\hline 204 & Evaluation for $C A D$ in patients being considered for kidney transplantation & Appropriate & 8 \\
\hline 205 & Evaluation for CAD in patients being considered for liver transplantation & Appropriate & 8 \\
\hline 206 & Evaluation for $C A D$ in patients being considered for lung transplantation & Appropriate & 8 \\
\hline
\end{tabular}

without clinical risk factors. For these patients, PET MPI is rarely appropriate.

Scenario 200: Intermediate-risk surgery and no clinical risk factors (Score 4 - May be Appropriate)

Scenario 201: Intermediate-risk surgery and $\geq 1$ clinical risk factor (Score 5 - May be Appropriate)

For patients undergoing intermediate-risk surgery, complications are increased compared with those for low-risk surgery and vary with the presence of clinical risk factors. Thus, PET MPI may be appropriate in these patients if it is expected to change management.

Scenario 202: Vascular surgery with no clinical risk factors (Score 7 - Appropriate)

Scenario 203: Vascular surgery with $\geq 1$ clinical risk factor (Score 8 - Appropriate)

For patients undergoing vascular surgery, complications are increased compared with those in patients undergoing intermediate-risk surgery and vary with the presence of clinical risk factors. Thus, PET MPI is appropriate in these patients if it is expected to change management.

Scenario 204: Evaluation for CAD in patients being considered for kidney transplantation (Score 8 - Appropriate)

Scenario 205: Evaluation for CAD in patients being considered for liver transplantation (Score 8 - Appropriate)

Scenario 206: Evaluation for CAD in patients being considered for lung transplantation (Score 8 - Appropriate)

For patients undergoing assessment for kidney, liver, or lung transplantation, preoperative screening for CAD with stress MPI is frequently used. Several studies have demonstrated the prognostic value of stress MPI for cardiac events in patients with end-stage renal failure who are being considered for renal transplantation (198-201).

\section{Summary of Recommendations}

PET MPI is rarely appropriate as a preoperative evaluation for noncardiac surgery in patients with moderate to good functional capacity, with no clinical risk factors, who are asymptomatic and it has been $<1$ y since functional stress testing with normal results, who are asymptomatic and it has been $<2$ y since CT or invasive angiography showing normal coronary morphology results, or since undergoing low-risk surgery.

If it is expected to change management, PET MPI may be appropriate for patients undergoing intermediate-risk surgery; it is appropriate for patients undergoing vascular surgery or who are being considered for kidney, liver, or lung transplantation.

\section{SECTION 11: DETERMINATION OF EXERCISE LEVEL BEFORE INITIATION OF EXERCISE PRESCRIPTION OR CARDIAC REHABILITATION}

\section{Introduction and Background}

Exercise testing is best for determining exercise capacity for exercise prescription or cardiac rehabilitation. Stress PET MPI uses pharmacologic stress and not exercise, providing detailed information about myocardial perfusion and ventricular function.

\section{Clinical Scenarios and AUC Scores}

Table 11 presents the following clinical scenarios and final AUC scores for the use of PET MPI to determine exercise level before the initiation of exercise prescription or cardiac rehabilitation.

Clinical Scenarios 207-210: Exercise Level Before Exercise Prescription or Cardiac Rehabilitation.

Scenario 207: Exercise prescription (Score 1 - Rarely Appropriate)

Scenario 208: Determination of exercise level before initiation of exercise prescription (Score 1 - Rarely Appropriate) 
TABLE 11

Clinical Scenarios for the Use of PET MPI to Determine Exercise Level Before Initiation of Exercise Prescription or Cardiac Rehabilitation

\begin{tabular}{lllc}
\hline Scenario no. & \multicolumn{1}{c}{ Description } & Appropriateness & Score \\
\hline 207 & Exercise prescription & Rarely Appropriate & 1 \\
208 & Determination of exercise level before initiation of exercise prescription & Rarely Appropriate & 1 \\
209 & Before the initiation of cardiac rehabilitation and able to exercise & Rarely Appropriate & 1 \\
210 & After revascularization (PCl or CABG) & Rarely Appropriate & 1 \\
\hline
\end{tabular}

Scenario 209: Before initiation of cardiac rehabilitation in patients able to exercise (Score 1 - Rarely Appropriate)

Scenario 210: After revascularization (PCI or CABG) (Score 1 Rarely Appropriate)

For these scenarios, exercise testing is preferred and stress PET MPI is rarely appropriate.

\section{Summary of Recommendations}

PET MPI is rarely appropriate in patients before exercise prescription, for determination of exercise level before initiation of exercise prescription, before initiation of cardiac rehabilitation, or after revascularization with $\mathrm{PCI}$ or $\mathrm{CABG}$.

\section{BENEFITS AND HARMS OF IMPLEMENTING THE AUC GUIDANCE}

These AUC imply a thorough and critical evaluation of the available literature on PET MPI and draw on the clinical experience of a range of experts skilled in the diagnosis and treatment of patients with suspected or known CAD, which may cause downstream flow limitations, and for patients with microvascular angina. The workgroup strove to choose specific scenarios for inclusion that were most clinically relevant and of broad interest. The AUC address the use of PET MPI in 11 sections, as follows: Symptomatic Patients with Suspected or Known CAD, Asymptomatic Patients (Without Symptoms or Ischemic Equivalent), Diagnosed Heart Failure (Resting LV Function Previously Assessed but No Prior CAD Evaluation), Evaluation of Patients with Known or Suspected Cardiac Sarcoidosis, Evaluation of Arrhythmias Without Ischemic Equivalent (No Prior Cardiac Evaluation), Syncope Without Ischemic Equivalent, Assessment of CMD in Symptomatic Patients, Specific Populations, Prior Testing or Procedures, Preoperative Evaluation for Noncardiac Surgery, and Determination of Exercise Level Before Initiation of Exercise Prescription or Cardiac Rehabilitation. Consequently, the AUC are a reflection of the knowledge and experience of experts as applied to common clinical scenarios involving PET MPI.

The benefit of these AUC, if used, is the standardization of an evidence- and expert-based approach to the diagnosis of patients with $\mathrm{CAD}$ and microvascular angina. This likely creates new avenues for auditing of outcomes and for collaborative research between centers.

Conversely, in the development of these AUC, the workgroup recognized that the application of PET MPI to identify and characterize the early subclinical and clinically manifest CAD process and CMD may vary at different centers, likely related to relative differences in the protocols, characteristics of positronemitting perfusion radiotracers such as ${ }^{13} \mathrm{~N}$-ammonia and ${ }^{82} \mathrm{Rb}$, installed hardware and software, and local expertise for interpretation and reporting of imaging studies. In this respect, local resources and expertise should be considered in making decisions about incorporating these AUC recommendations. The workgroup also acknowledges that there are likely additional scenarios not addressed by these AUC in which PET MPI may be entirely justified. This document therefore does not aim and is not able to include all clinical scenarios in which PET MPI should be applied.

The assessment of LV wall motion at peak stress and at rest with the gating portion of the PET MPI, in conjunction with perfusion and flow measurements, affords identification and characterization of regional or global ischemia and downstream flow-limiting effects of left main or multivessel CAD that may be missed by conventional MPI. In addition, the concurrent quantification of hyperemic MBF and MFR with PET MPI affords the unique and noninvasive identification of $\mathrm{CMD}$ as an underlying cause for microvascular angina pectoris amenable to various treatment options. The identification and severity assessment of CMD also adds incremental outcome information that further improves prognostication in patients with cardiovascular risk. Implementing these novel parameters (wall motion assessment at peak stress and MBF quantification) in measures of myocardial perfusion with PET affords a new comprehensive assessment of subclinical and clinically manifest coronary vasculopathy. This approach expands the scope of conventional MPI to a variety of important new clinical scenarios in cardiovascular disease that is expected to further improve patient care in a cost-effective manner.

\section{IMPLEMENTATION OF THE AUC GUIDANCE}

To develop broad-based multidisciplinary clinical guidance documents, SNMMI has been working with several other medical specialty societies. It is hoped that this collaboration will foster the acceptance and adoption of this guidance by other specialties. SNMMI has developed a multipronged approach to disseminate AUC for PET MPI to all relevant stakeholders, including referring physicians, nuclear medicine physicians, and patients. The dissemination and implementation tactics will include a mix of outreach and educational activities targeted to each of these audiences. SNMMI will create case studies for its members, as well as for referring physicians, and make them available via online modules and webinars. These cases will cover the appropriate clinical scenarios for the use of PET MPI studies. Related resources such as the systematic review supporting the development of these AUC, a list of upcoming education events on the AUC, factsheets, and other didactic materials will be made available on the SNMMI website. Live sessions will be held at the SNMMI annual and midwinter meetings, as well as at other relevant professional society meetings of referring physicians to highlight the importance and application of 
these AUC. SNMMI also aims to create a mobile application for these AUC for both Apple and Android platforms.

\section{ACKNOWLEDGMENTS}

The workgroup acknowledges staff support from the Pacific Northwest Evidence-Based Practice Center of Oregon Health and Science University (Roger Chou, MD, FACP - Principal Investigator; Tamara P. Cheney, MD - Research Associate; Ian Blazina, MPH - Research Associate; Sara Grusing, BA - Research Assistant; Christina Bougatsos, MPH - Research Associate and Project Manager; Tracy Dana, MLS - Research Librarian; Elaine Graham, MLS - EPC Operations Manager, provided project oversight).

\section{APPENDIX A: WORKGROUP MEMBERS AND EXTERNAL REVIEWERS}

\section{Workgroup}

The members of the workgroup are Thomas H. Schindler, MD, Washington University in St. Louis, St. Louis, MO (SNMMI); Timothy Bateman, MD, Saint Luke's Health System, Kansas City, MO (SNMMI); Rob Beanlands, MD, FRCPC, FACC, University of Ottawa Heart Institute, Ottawa, Canada (ASNC, CCS, ACC, SNNMI, CSNC); Daniel S. Berman, MD, Cedars-Sinai Medical Center, Los Angeles, CA (SNMMI, ACC, SCCT); Panithaya Chareonthaitawee, MD, Mayo Clinic, Rochester, MN (SNMMI, ASNC); Lorraine E. De Blanche, MD, Veterans Association Health System North Texas, Dallas, TX (ACNM); Marcelo F. Di Carli, MD, Brigham \& Women's Hospital, Boston, MA (AHA, SNMMI); Vasken Dilsizian, MD, University of Maryland Medical Center, Baltimore, MD (SNMMI, ASNC); Sharmila Dorbala, MD, MPH, Brigham and Women's Hospital, Boston, MA (SNMMI); Robert J. Gropler, MD, Washington University in St. Louis, MO (SNMMI); Venkatesh L. Murthy, MD, PhD, University of Michigan, Ann Arbor, MI (SNMMI); Terrence Ruddy, MD, FRCPC, FACC, University of Ottawa Heart Institute, Ottawa, Ontario, Canada (SNMMI, ASNC, CCS, CSNC); Ronald G. Schwartz, MD, MS, FACC, FAHA, University of Rochester Medical Center, Rochester, NY; Leslee Shaw, MD, Emory University, Atlanta, GA (ACC, SCCT); Prem Soman, MD, PhD, FRCP (UK), FACC, University of Pittsburgh Medical Center, Pittsburgh, PA (SNMMI, ACC, ASNC); David E. Winchester, MD, University of Florida, Gainesville, FL (ACP); Hein Verberne, MD, PhD, Academic Medical Center, Amsterdam, Netherlands (EANM).

\section{External Reviewers}

The external (peer) reviewers are Frank M. Bengel, MD, Hannover Medical School, Hannover, Germany; Henry Gewirtz, MD, Massachusetts General Hospital, Harvard Medical School, Boston MA; Fabien Hyafil, MD, Richar University Hospital, Paris, France; Juhani Knuuti, MD, University of Turku, Turku PET Centre, Turku, Finland; René R. Bevag Packard, MD, PhD, University of California, Los Angeles, CA; Heinrich R. Schelbert, $\mathrm{MD}, \mathrm{PhD}$, University of California, Los Angeles, CA; Nagara Tamaki, MD, PhD, Kyoto Prefectural University of Medicine, Kyoto, Japan; Mark Travin, Montefiore Medical Center, Albert Einstein College of Medicine, New York, NY.

\section{SNMM}

The supporting staff from SNMMI are Sukhjeet Ahuja, MD, MPH, Director, Health Policy \& Quality Department; Teresa Ellmer, MIS, CNMT, Senior Program Manager, Health Policy \& Quality Department; Julie Kauffman, Program Manager, Health Policy \& Quality Department.

\section{APPENDIX B: DEFINITION OF TERMS AND ACRONYMS}

$$
\begin{aligned}
& \mathrm{ACC}=\text { American College of Cardiology } \\
& \text { ACE-I }=\text { angiotensin-converting enzyme inhibitor } \\
& \mathrm{ACP}=\text { American College of Physicians } \\
& \text { ACS }=\text { acute coronary syndrome } \\
& \mathrm{AF}=\text { atrial fibrillation } \\
& \mathrm{AHA}=\text { American Heart Association } \\
& \mathrm{ARB}=\text { angiotensin type } 2 \text { receptor blocker } \\
& \text { ASCVD }=\text { atherosclerotic cardiovascular disease } \\
& \mathrm{ASNC}=\text { American Society of Nuclear Cardiology } \\
& \mathrm{AUC}=\text { appropriate use criteria } \\
& \mathrm{BMI}=\text { body mass index } \\
& \mathrm{CABG}=\text { coronary artery bypass graft } \\
& \mathrm{CAC}=\text { coronary artery calcium } \\
& \mathrm{CAD}=\text { coronary artery disease } \\
& \mathrm{CAV}=\text { cardiac allograft vasculopathy } \\
& \mathrm{CCS}=\text { Canadian Cardiovascular Society } \\
& \mathrm{CCT}=\text { cardiac } \mathrm{CT} \\
& \mathrm{CCTA}=\text { coronary } \mathrm{CT} \text { angiography } \\
& \mathrm{CMD}=\text { coronary microvascular disease } \\
& \mathrm{COI}=\text { conflict of interest }
\end{aligned}
$$

COVADIS $=$ Coronary Vasomotion Disorders International Study Group

$$
\begin{aligned}
& \text { CT }=\text { CT } \\
& \text { DTS = Duke treadmill score } \\
& \text { EANM = European Association of Nuclear Medicine } \\
& \text { ECG = electrocardiogram } \\
& \text { ED = emergency department } \\
& \text { ETT = exercise treadmill test } \\
& \text { GDMT = guideline-directed medical therapy } \\
& \text { HCM = hypertrophic cardiomyopathy } \\
& \text { HFpEF = heart failure with preserved ejection fraction } \\
& \text { ICA = invasive coronary angiography } \\
& \text { LBBB = left bundle branch block } \\
& \text { LV = left ventricular } \\
& \text { LVEF = left ventricular ejection fraction } \\
& \text { MBF = myocardial blood flow }
\end{aligned}
$$


$\mathrm{MET}=$ metabolic equivalent of task

MFR $=$ myocardial flow reserve

MI = myocardial infarction

MPI = myocardial perfusion imaging

MPR $=$ myocardial perfusion reserve

Non-STEMI = non-ST-elevation myocardial infarction

PCI $=$ percutaneous coronary intervention

$\mathrm{PVC}=$ premature ventricular contraction

SCCT $=$ Society of Cardiovascular CT

SNMMI $=$ Society of Nuclear Medicine and Molecular Imaging

$\mathrm{Tn}=$ troponin

$\mathrm{VF}=$ ventricular fibrillation

$\mathrm{VT}=$ ventricular tachycardia

\section{APPENDIX C: DISCLOSURES AND CONFLICTS OF INTEREST (COIS)}

SNMMI rigorously attempted to avoid any actual, perceived, or potential COIs that might have arisen as a result of an outside relationship or personal interest on the part of the workgroup members or external reviewers. Workgroup members were required to provide disclosure statements of all relationships that might be perceived as real or potential COIs. These statements were reviewed and discussed by the workgroup chair and SNMMI staff and were updated and reviewed by an objective third party at the beginning of every workgroup meeting or teleconference. The disclosures of the workgroup members can be found in Table 12 . A COI was defined as a relationship with industry-including consulting, speaking, research, and nonresearch activities-that exceeds $\$ 5,000$ in funding over the previous or upcoming 12-mo period. In addition, if an external reviewer was either the principal investigator of a study or another key member of the study personnel, that person's participation in the review was considered

TABLE 12

Relationships with Industry and Other Entities

\begin{tabular}{|c|c|}
\hline Workgroup member & Reported relationships \\
\hline Thomas H. Schindler & $\begin{array}{l}\text { - Siemens, Validation of IQ - SPECT/CT for Cardiac Perfusion } \\
\text { - GE Healthcare, Flupiridaz, PET Perfusion Study }\end{array}$ \\
\hline Timothy M. Bateman & $\begin{array}{l}\text { - License/Patent, ExSPECT } \\
\text { - Imagen Pro/MD/3D/Q }\end{array}$ \\
\hline Rob Beanlands & $\begin{array}{l}\text { - Lantheus Medical Imaging, Consultant } \\
\text { - Lantheus Medical Imaging, Research/Grants } \\
\text { - Jubilant DRAX Image, Consultant } \\
\text { - Jubilant DRAX Image, Research/Grants }\end{array}$ \\
\hline Daniel S. Berman & $\begin{array}{l}\text { - Bayer Pharmaceuticals, Grant, MRI } \\
\text { - Cedars-Sinai Medical, Royalties, Software }\end{array}$ \\
\hline Panithaya Chareonthaitawee & - None \\
\hline Lorraine De Blanche & - None \\
\hline Marcelo Di Carli & - Spectrum Dynamics, Clinical Trial, SPECT MPI \\
\hline Vasken Dilsizian & - GE Healthcare, Research Grant, Cardiac Innervation \\
\hline Sharmila Dorbala & - None \\
\hline Robert Gropler & $\begin{array}{l}\text { - Sanofi, Scientific Advisory Meeting, PET MPI } \\
\text { - Biomedical Systems, Consultant, Read EKGs } \\
\text { - Bayer, Research Grant, MR MPI }\end{array}$ \\
\hline Venkatesh Murthy & $\begin{array}{l}\text { - Ionetix, Scientific Advisor, Cyclotrons } \\
\text { - Siemens, Research Grant, Cardiac SPECT } \\
\text { - General Electric, Stock } \\
\text { - Cardinal Health, Stock }\end{array}$ \\
\hline Terrence D. Ruddy & $\begin{array}{l}\text { - GE Healthcare, Clinical Trial Agreement, Myocardial Blood Flow } \\
\text { - Advanced Accelerator Applications, Clinical Trial Agreement, Apoplosis Imaging }\end{array}$ \\
\hline Ronald G. Schwartz & - Astellas, Speaker \\
\hline Leslee Shaw & - None \\
\hline Prem Soman & $\begin{array}{l}\text { - Astellas, Grant funding } \\
\text { - Alnylam, Advisory board }\end{array}$ \\
\hline Hein J. Verberne & - None \\
\hline David Winchester & - Roche Diagnostics, Grant, Biomarkers \\
\hline
\end{tabular}


likely to present a COI. All reviewers were asked about any potential COI. A COI was also considered likely if an external reviewer or workgroup member was either the principal investigator or a key member of a study directly related to the content of this AUC document. All external reviewers were asked about any potential COI.

\section{APPENDIX D: PUBLIC COMMENTARY}

The workgroup solicited information from all communities through the SNMMI website and through direct solicitation of SNMMI members. The comments and input helped to shape the development of these AUC on the use of nuclear medicine in PET$\mathrm{MCI}$ in patients with suspected CAD or microvascular angina or with known CAD.

\section{REFERENCES}

1. Bateman TM, Dilsizian V, Beanlands RS, DePuey EG, Heller GV, Wolinsky DA. American Society of Nuclear Cardiology and Society of Nuclear Medicine and Molecular Imaging joint position statement on the clinical indications for myocardial perfusion PET. J Nucl Cardiol. 2016;23: 1227-1231.

2. Schindler TH, Schelbert HR, Quercioli A, Dilsizian V. Cardiac PET imaging for the detection and monitoring of coronary artery disease and microvascular health. JACC Cardiovasc Imaging. 2010;3:623-640.

3. Taqueti VR, Di Carli MF. Coronary microvascular disease pathogenic mechanisms and therapeutic options: JACC state-of-the-art review. J Am Coll Cardiol. 2018;72:2625-2641.

4. Patel KK, Spertus JA, Chan PS, et al. Myocardial blood flow reserve assessed by positron emission tomography myocardial perfusion imaging identifies patients with a survival benefit from early revascularization. Eur Heart J. 2020;41:759-768

5. Patel KK, Spertus JA, Chan PS, et al. Extent of myocardial ischemia on positron emission tomography and survival benefit with early revascularization. J Am Coll Cardiol. 2019;74:1645-1654.

6. Gould KL, Johnson NP, Bateman TM, et al. Anatomic versus physiologic assessment of coronary artery disease: role of coronary flow reserve, fractional flow reserve, and positron emission tomography imaging in revascularization decision-making. J Am Coll Cardiol. 2013;62:1639-1653.

7. Gould KL, Johnson NP, Roby AE, et al. Regional, artery-specific thresholds of quantitative myocardial perfusion by PET associated with reduced myocardial infarction and death after revascularization in stable coronary artery disease. J Nucl Med. 2019;60:410-417.

8. Naya M, Murthy VL, Taqueti VR, et al. Preserved coronary flow reserve effectively excludes high-risk coronary artery disease on angiography. $\mathrm{J} \mathrm{Nucl}$ Med. 2014;55:248-255.

9. Schindler TH. Myocardial blood flow: putting it into clinical perspective. J Nucl Cardiol. 2016;23:1056-1071.

10. Valenta I, Quercioli A, Schindler TH. Diagnostic value of PET-measured longitudinal flow gradient for the identification of coronary artery disease. JACC Cardiovasc Imaging. 2014;7:387-396.

11. Parkash R, deKemp RA, Ruddy TD, et al. Potential utility of rubidium 82 PET quantification in patients with 3-vessel coronary artery disease. $\mathrm{J} \mathrm{Nucl} \mathrm{Cardiol.}$ 2004:11:440-449.

12. Protecting Access to Medicare Act of 2014, Pub L No. 113-93, 128 Stat 1040 (2014).

13. Schindler TH, Dilsizian V. Coronary microvascular dysfunction: clinical considerations and noninvasive diagnosis. JACC Cardiovasc Imaging. 2020;13: 140-155.

14. Taqueti VR, Everett BM, Murthy VL, et al. Interaction of impaired coronary flow reserve and cardiomyocyte injury on adverse cardiovascular outcomes in patients without overt coronary artery disease. Circulation. 2015;131: 528-535.

15. Schindler TH, Nitzsche EU, Schelbert HR, et al. Positron emission tomography-measured abnormal responses of myocardial blood flow to sympathetic stimulation are associated with the risk of developing cardiovascular events. $J$ Am Coll Cardiol. 2005;45:1505-1512.

16. Tio RA, Dabeshlim A, Siebelink HM, et al. Comparison between the prognostic value of left ventricular function and myocardial perfusion reserve in patients with ischemic heart disease. J Nucl Med. 2009;50:214-219.
17. Van Tosh A, Supino PG, Nichols KJ, Garza D, Horowitz SF, Reichek N. Prognosis of a normal positron emission tomography ${ }^{82} \mathrm{Rb}$ myocardial perfusion imaging study in women with no history of coronary disease. Cardiology. 2010;117:301-306

18. Williams BA, Dorn JM, LaMonte MJ, et al. Evaluating the prognostic value of positron-emission tomography myocardial perfusion imaging using automated software to calculate perfusion defect size. Clin Cardiol. 2012;35:E14-E21.

19. Hendel RC, Patel MR, Allen JM, et al. Appropriate use of cardiovascular technology: 2013 ACCF appropriate use criteria methodology update: a report of the American College of Cardiology Foundation appropriate use criteria task force. J Am Coll Cardiol. 2013;61:1305-1317.

20. Fitch K, Bernstein SJ, Aguilar MD, Burnand B. The RAND/UCLA Appropriateness Method User's Manual. Santa Monica, CA: RAND; 2001.

21. Institute of Medicine. Clinical Practice Guidelines We Can Trust. Washington, DC: National Academies Press; 2011.

22. Pacific Northwest Evidence-based Practice Center. Systematic Review: Positron Emission Tomography Myocardial Perfusion Imaging. Portland, OR: Oregon Health and Science University; 2018.

23. Shea BJ, Bouter LM, Peterson J, et al. External validation of a measurement tool to assess systematic reviews (AMSTAR). PLoS One. 2007;2:e1350.

24. Whiting PF, Rutjes AW, Westwood ME, et al. QUADAS-2: a revised tool for the quality assessment of diagnostic accuracy studies. Ann Intern Med. 2011;155:529-536.

25. AQA Principles for Appropriateness Criteria. London, U.K.: Assessment and Qualifications Alliance; 2009.

26. Dilsizian V, Bacharach SL, Beanlands RS, et al. ASNC imaging guidelines/ SNMMI procedure standard for positron emission tomography (PET) nuclear cardiology procedures. J Nucl Cardiol. 2016;23:1187-1226.

27. Klocke FJ, Baird MG, Lorell BH, et al. ACC/AHA/ASNC guidelines for the clinical use of cardiac radionuclide imaging-executive summary: a report of the American College of Cardiology/American Heart Association Task Force on Practice Guidelines (ACC/AHA/ASNC Committee to Revise the 1995 Guidelines for the Clinical Use of Cardiac Radionuclide Imaging). Circulation. 2003;108:1404-1418.

28. Wolk MJ, Bailey SR, Doherty JU, et al. ACCF/AHA/ASE/ASNC/HFSA/HRS/ SCAI/SCCT/SCMR/STS 2013 multimodality appropriate use criteria for the detection and risk assessment of stable ischemic heart disease: a report of the American College of Cardiology Foundation Appropriate Use Criteria Task Force, American Heart Association, American Society of Echocardiography, American Society of Nuclear Cardiology, Heart Failure Society of America, Heart Rhythm Society, Society for Cardiovascular Angiography and Interventions, Society of Cardiovascular Computed Tomography, Society for Cardiovascular Magnetic Resonance, and Society of Thoracic Surgeons. J Am Coll Cardiol. 2014;63:380-406.

29. Dorbala S, Di Carli MF, Beanlands RS, et al. Prognostic value of stress myocardial perfusion positron emission tomography: results from a multicenter observational registry. J Am Coll Cardiol. 2013;61:176-184.

30. Yoshinaga K, Chow BJ, Williams $\mathrm{K}$, et al. What is the prognostic value of myocardial perfusion imaging using rubidium- 82 positron emission tomography? J Am Coll Cardiol. 2006;48:1029-1039.

31. Lertsburapa K, Ahlberg AW, Bateman TM, et al. Independent and incremental prognostic value of left ventricular ejection fraction determined by stress gated rubidium 82 PET imaging in patients with known or suspected coronary artery disease. J Nucl Cardiol. 2008;15:745-753.

32. Sharir T, Germano G, Kang X, et al. Prediction of myocardial infarction versus cardiac death by gated myocardial perfusion SPECT: risk stratification by the amount of stress-induced ischemia and the poststress ejection fraction. $\mathrm{J} \mathrm{Nucl}$ Med. 2001;42:831-837.

33. Parker MW, Iskandar A, Limone B, et al. Diagnostic accuracy of cardiac positron emission tomography versus single photon emission computed tomography for coronary artery disease: a bivariate meta-analysis. Circ Cardiovasc Imaging. 2012;5:700-707.

34. Bateman TM, Heller GV, McGhie AI, et al. Diagnostic accuracy of rest/stress ECG-gated Rb-82 myocardial perfusion PET: comparison with ECG-gated Tc99m sestamibi SPECT. J Nucl Cardiol. 2006;13:24-33.

35. Berman DS, Kang X, Slomka PJ, et al. Underestimation of extent of ischemia by gated SPECT myocardial perfusion imaging in patients with left main coronary artery disease. J Nucl Cardiol. 2007;14:521-528.

36. Dorbala S, Vangala D, Sampson U, Limaye A, Kwong R, Di Carli MF. Value of vasodilator left ventricular ejection fraction reserve in evaluating the magnitude of myocardium at risk and the extent of angiographic coronary artery disease: a ${ }^{82} \mathrm{Rb}$ PET/CT study. J Nucl Med. 2007;48:349-358.

37. Lima RS, Watson DD, Goode AR, et al. Incremental value of combined perfusion and function over perfusion alone by gated SPECT myocardial perfusion 
imaging for detection of severe three-vessel coronary artery disease. J Am Coll Cardiol. 2003;42:64-70.

38. Mc Ardle BA, Dowsley TF, deKemp RA, Wells GA, Beanlands RS. Does rubidium-82 PET have superior accuracy to SPECT perfusion imaging for the diagnosis of obstructive coronary disease?: a systematic review and metaanalysis. J Am Coll Cardiol. 2012;60:1828-1837.

39. Murthy VL, Bateman TM, Beanlands RS, et al. Clinical quantification of myocardial blood flow using PET: joint position paper of the SNMMI Cardiovascular Council and the ASNC. J Nucl Med. 2018;59:273-293.

40. Danad I, Raijmakers PG, Driessen RS, et al. Comparison of coronary CT angiography, SPECT, PET, and hybrid imaging for diagnosis of ischemic heart disease determined by fractional flow reserve. JAMA Cardiol. 2017;2:11001107.

41. Driessen RS, Danad I, Stuijfzand WJ, et al. Comparison of coronary computed tomography angiography, fractional flow reserve, and perfusion imaging for ischemia diagnosis. J Am Coll Cardiol. 2019;73:161-173.

42. Hajjiri MM, Leavitt MB, Zheng H, Spooner AE, Fischman AJ, Gewirtz H. Comparison of positron emission tomography measurement of adenosine-stimulated absolute myocardial blood flow versus relative myocardial tracer content for physiological assessment of coronary artery stenosis severity and location. JACC Cardiovasc Imaging. 2009;2:751-758.

43. Johnson NP, Kirkeeide RL, Gould KL. Is discordance of coronary flow reserve and fractional flow reserve due to methodology or clinically relevant coronary pathophysiology? JACC Cardiovasc Imaging. 2012;5:193-202.

44. Kajander S, Joutsiniemi E, Saraste M, et al. Cardiac positron emission tomography/computed tomography imaging accurately detects anatomically and functionally significant coronary artery disease. Circulation. 2010;122:603-613.

45. Valenta I, Antoniou A, Marashdeh W, et al. PET-measured longitudinal flow gradient correlates with invasive fractional flow reserve in CAD patients. Eur Heart J Cardiovasc Imaging. 2017;18:538-548.

46. Ziadi MC, Dekemp RA, Williams KA, et al. Impaired myocardial flow reserve on rubidium-82 positron emission tomography imaging predicts adverse outcomes in patients assessed for myocardial ischemia. J Am Coll Cardiol. 2011;58:740-748.

47. Gupta A, Taqueti VR, van de Hoef TP, et al. Integrated noninvasive physiological assessment of coronary circulatory function and impact on cardiovascular mortality in patients with stable coronary artery disease. Circulation. 2017;136: 2325-2336.

48. Murthy VL, Naya M, Foster CR, et al. Association between coronary vascular dysfunction and cardiac mortality in patients with and without diabetes mellitus. Circulation. 2012;126:1858-1868.

49. Braunwald E, Antman EM, Beasley JW, et al. ACC/AHA guidelines for the management of patients with unstable angina and non-st-segment elevation myocardial infarction: a report of the American College of Cardiology/American Heart Association Task Force on Practice Guidelines (Committee on the Management of Patients With Unstable Angina). J Am Coll Cardiol. 2000;36: 970-1062.

50. Pollack CV Jr, Braunwald E. 2007 update to the ACC/AHA guidelines for the management of patients with unstable angina and non-ST-segment elevation myocardial infarction: implications for emergency department practice. Ann Emerg Med. 2008;51:591-606.

51. Duvall WL, Savino JA, Levine EJ, et al. A comparison of coronary CTA and stress testing using high-efficiency SPECT MPI for the evaluation of chest pain in the emergency department. J Nucl Cardiol. 2014;21:305-318.

52. Duvall WL, Wijetunga MN, Klein TM, et al. Stress-only Tc-99m myocardial perfusion imaging in an emergency department chest pain unit. J Emerg Med. 2012;42:642-650.

53. Einstein AJ, Johnson LL, DeLuca AJ, et al. Radiation dose and prognosis of ultra-low-dose stress-first myocardial perfusion SPECT in patients with chest pain using a high-efficiency camera. J Nucl Med. 2015;56:545-551.

54. Nabi F, Chang SM, Xu J, Gigliotti E, Mahmarian JJ. Assessing risk in acute chest pain: the value of stress myocardial perfusion imaging in patients admitted through the emergency department. J Nucl Cardiol. 2012;19:233-243.

55. Stone NJ, Robinson JG, Lichtenstein AH, et al. 2013 ACC/AHA guideline on the treatment of blood cholesterol to reduce atherosclerotic cardiovascular risk in adults: a report of the American College of Cardiology/American Heart Association Task Force on Practice Guidelines. J Am Coll Cardiol. 2014; 63(25 Pt B):2889-2934.

56. Berman DS, Wong ND, Gransar H, et al. Relationship between stress-induced myocardial ischemia and atherosclerosis measured by coronary calcium tomography. J Am Coll Cardiol. 2004;44:923-930.

57. Chang SM, Nabi F, Xu J, et al. The coronary artery calcium score and stress myocardial perfusion imaging provide independent and complementary prediction of cardiac risk. J Am Coll Cardiol. 2009;54:1872-1882.
58. Chang SM, Nabi F, Xu J, et al. Value of CACS compared with ETT and myocardial perfusion imaging for predicting long-term cardiac outcome in asymptomatic and symptomatic patients at low risk for coronary disease: clinical implications in a multimodality imaging world. JACC Cardiovasc Imaging. 2015;8:134-144.

59. Clerc OF, Possner M, Maire R, et al. Association of left bundle branch block with obstructive coronary artery disease on coronary CT angiography: a casecontrol study. Eur Heart J Cardiovasc Imaging. 2016;17:765-771.

60. Fleisher LA, Fleischmann KE, Auerbach AD, et al. 2014 ACC/AHA guideline on perioperative cardiovascular evaluation and management of patients undergoing noncardiac surgery: executive summary: a report of the American College of Cardiology/American Heart Association Task Force on Practice Guidelines. Circulation. 2014;130:2215-2245.

61. Hendel RC, Berman DS, Di Carli MF, et al. ACCF/ASNC/ACR/AHA/ASE/ SCCT/SCMR/SNM 2009 appropriate use criteria for cardiac radionuclide imaging: a report of the American College of Cardiology Foundation Appropriate Use Criteria Task Force, the American Society of Nuclear Cardiology, the American College of Radiology, the American Heart Association, the American Society of Echocardiography, the Society of Cardiovascular Computed Tomography, the Society for Cardiovascular Magnetic Resonance, and the Society of Nuclear Medicine. Circulation. 2009;119:e561-e587.

62. Wolk MJ, Bailey SR, Doherty JU, et al. Multimodality Writing Group for Stable Ischemic Heart Disease. ACCF/AHA/ASE/ASNC/HFSA/HRS/SCAI/ SCCT/SCMR/STS 2013 multimodality appropriate use criteria for the detection and risk assessment of stable ischemic heart disease: a report of the American College of Cardiology Foundation Appropriate Use Criteria Task Force, American Heart Association, American Society of Echocardiography, American Society of Nuclear Cardiology, Heart Failure Society of America, Heart Rhythm Society, Society for Cardiovascular Angiography and Interventions, Society of Cardiovascular Computed Tomography, Society for Cardiovascular Magnetic Resonance, and Society of Thoracic Surgeons. J Card Fail. 2014;20:65-90.

63. Naya M, Murthy VL, Foster CR, et al. Prognostic interplay of coronary artery calcification and underlying vascular dysfunction in patients with suspected coronary artery disease. J Am Coll Cardiol. 2013;61:2098-2106.

64. Rozanski A, Gransar H, Wong ND, et al. Use of coronary calcium scanning for predicting inducible myocardial ischemia: influence of patients' clinical presentation. J Nucl Cardiol. 2007;14:669-679.

65. Schenker MP, Dorbala S, Hong EC, et al. Interrelation of coronary calcification, myocardial ischemia, and outcomes in patients with intermediate likelihood of coronary artery disease: a combined positron emission tomography/computed tomography study. Circulation. 2008;117:1693-1700.

66. Trinder M, Li X, DeCastro ML, et al. Risk of premature atherosclerotic disease in patients with monogenic versus polygenic familial hypercholesterolemia. J Am Coll Cardiol. 2019;74:512-522.

67. Askew JW, Miller TD, Hodge DO, Gibbons RJ. The value of myocardial perfusion single-photon emission computed tomography in screening asymptomatic patients with atrial fibrillation for coronary artery disease. J Am Coll Cardiol. 2007;50:1080-1085.

68. Knuuti J, Wijns W, Saraste A, et al. 2019 ESC guidelines for the diagnosis and management of chronic coronary syndromes. Eur Heart J. 2020;41:407-477.

69. Majmudar MD, Murthy VL, Shah RV, et al. Quantification of coronary flow reserve in patients with ischaemic and non-ischaemic cardiomyopathy and its association with clinical outcomes. Eur Heart J Cardiovasc Imaging. 2015; 16:900-909.

70. Neglia D, Michelassi C, Trivieri MG, et al. Prognostic role of myocardial blood flow impairment in idiopathic left ventricular dysfunction. Circulation. 2002; 105:186-193.

71. Yancy CW, Jessup M, Bozkurt B, et al. 2013 ACCF/AHA guideline for the management of heart failure: executive summary: a report of the American College of Cardiology Foundation/American Heart Association Task Force on practice guidelines. Circulation. 2013;128:1810-1852.

72. Bravo PE, Bergmark BA, Vita T, et al. Diagnostic and prognostic value of myocardial blood flow quantification as non-invasive indicator of cardiac allograft vasculopathy. Eur Heart J. 2018;39:316-323.

73. Chih S, Chong AY, Erthal F, et al. PET assessment of epicardial intimal disease and microvascular dysfunction in cardiac allograft vasculopathy. J Am Coll Cardiol. 2018;71:1444-1456.

74. Mc Ardle BA, Davies RA, Chen L, et al. Prognostic value of rubidium-82 positron emission tomography in patients after heart transplant. Circ Cardiovasc Imaging. 2014;7:930-937.

75. Miller RJH, Manabe O, Tamarappoo BK, et al. Comparative prognostic and diagnostic value of myocardial blood flow and myocardial flow reserve after cardiac transplant. J Nucl Med. 2020;61:249-255. 
76. Lentine KL, Costa SP, Weir MR, et al. Cardiac disease evaluation and management among kidney and liver transplantation candidates: a scientific statement from the American Heart Association and the American College of Cardiology Foundation: endorsed by the American Society of Transplant Surgeons, American Society of Transplantation, and National Kidney Foundation. Circulation. 2012;126:617-663.

77. Basso C, Maron BJ, Corrado D, Thiene G. Clinical profile of congenital coronary artery anomalies with origin from the wrong aortic sinus leading to sudden death in young competitive athletes. J Am Coll Cardiol. 2000;35: 1493-1501

78. Brothers JA, McBride MG, Seliem MA, et al. Evaluation of myocardial ischemia after surgical repair of anomalous aortic origin of a coronary artery in a series of pediatric patients. J Am Coll Cardiol. 2007;50:2078-2082.

79. McCrindle BW, Rowley AH, Newburger JW, et al. Diagnosis, treatment, and long-term management of Kawasaki disease: a scientific statement for health professionals from the American Heart Association. Circulation. 2017;135: e927-e999.

80. Velazquez EJ, Lee KL, Jones RH, et al. Coronary-artery bypass surgery in patients with ischemic cardiomyopathy. N Engl J Med. 2016;374:1511-1520.

81. Panza JA, Ellis AM, Al-Khalidi HR, et al. Myocardial viability and long-term outcomes in ischemic cardiomyopathy. N Engl J Med. 2019;381:739-748.

82. Abraham A, Nichol G, Williams KA, et al. ${ }^{18}$ F-FDG PET imaging of myocardial viability in an experienced center with access to ${ }^{18} \mathrm{~F}-\mathrm{FDG}$ and integration with clinical management teams: the Ottawa-FIVE substudy of the PARR 2 trial. J Nucl Med. 2010;51:567-574.

83. Ling LF, Marwick TH, Flores DR, et al. Identification of therapeutic benefit from revascularization in patients with left ventricular systolic dysfunction: inducible ischemia versus hibernating myocardium. Circ Cardiovasc Imaging. 2013;6:363-372.

84. Konerman MC, Greenberg JC, Kolias TJ, et al. Reduced myocardial flow reserve is associated with diastolic dysfunction and decreased left atrial strain in patients with normal ejection fraction and epicardial perfusion. J Card Fail. 2018;24:90-100.

85. Taqueti VR, Solomon SD, Shah AM, et al. Coronary microvascular dysfunction and future risk of heart failure with preserved ejection fraction. Eur Heart J. 2018;39:840-849.

86. Chareonthaitawee P, Beanlands RS, Chen W, et al. Joint SNMMI-ASNC expert consensus document on the role of ${ }^{18} \mathrm{~F}-\mathrm{FDG}$ PET/CT in cardiac sarcoid detection and therapy monitoring. J Nucl Med. 2017;58:1341-1353.

87. Slart RH, Glaudemans A, Lancellotti P, et al. A joint procedural position statement on imaging in cardiac sarcoidosis: from the Cardiovascular and Inflammation \& Infection Committees of the European Association of Nuclear Medicine, the European Association of Cardiovascular Imaging, and the American Society of Nuclear Cardiology. J Nucl Cardiol. 2018;25:298-319.

88. Broustet JP, Douard H, Mora B. Exercise testing in arrhythmias of idiopathic mitral valve prolapse. Eur Heart J. 1987;8(suppl D):37-42.

89. Ng GA. Treating patients with ventricular ectopic beats. Heart. 2006;92:17071712 .

90. Kennedy HL, Whitlock JA, Sprague MK, Kennedy LJ, Buckingham TA, Goldberg RJ. Long-term follow-up of asymptomatic healthy subjects with frequent and complex ventricular ectopy. $N$ Engl J Med. 1985;312:193-197.

91. Southall DP, Johnston F, Shinebourne EA, Johnston PG. 24-Hour electrocardiographic study of heart rate and rhythm patterns in population of healthy children. Br Heart J. 1981;45:281-291.

92. Camm AJ, Evans KE, Ward DE, Martin A. The rhythm of the heart in active elderly subjects. Am Heart J. 1980;99:598-603.

93. Lown B, Fakhro AM, Hood WB Jr, Thorn GW. The coronary care unit: new perspectives and directions. JAMA. 1967;199:188-198.

94. Duffee DF, Shen WK, Smith HC. Suppression of frequent premature ventricular contractions and improvement of left ventricular function in patients with presumed idiopathic dilated cardiomyopathy. Mayo Clin Proc. 1998;73:430-433.

95. Yarlagadda RK, Iwai S, Stein KM, et al. Reversal of cardiomyopathy in patients with repetitive monomorphic ventricular ectopy originating from the right ventricular outflow tract. Circulation. 2005;112:1092-1097.

96. Baman TS, Lange DC, Ilg KJ, et al. Relationship between burden of premature ventricular complexes and left ventricular function. Heart Rhythm. 2010;7:865869.

97. El Kadri M, Yokokawa M, Labounty T, et al. Effect of ablation of frequent premature ventricular complexes on left ventricular function in patients with nonischemic cardiomyopathy. Heart Rhythm. 2015;12:706-713.

98. Dukes JW, Dewland TA, Vittinghoff E, et al. Ventricular ectopy as a predictor of heart failure and death. J Am Coll Cardiol. 2015;66:101-109.
99. Kirchhof P, Benussi S, Kotecha D, et al. 2016 ESC guidelines for the management of atrial fibrillation developed in collaboration with EACTS. Eur Heart J. 2016;37:2893-2962.

100. Shen WK, Sheldon RS, Benditt DG, et al. 2017 ACC/AHA/HRS guideline for the evaluation and management of patients with syncope: a report of the American College of Cardiology/American Heart Association Task Force on Clinical Practice Guidelines and the Heart Rhythm Society. Circulation. 2017;136:e60e122.

101. Patel MR, Peterson ED, Dai D, et al. Low diagnostic yield of elective coronary angiography. N Engl J Med. 2010;362:886-895.

102. Ong P, Camici PG, Beltrame JF, et al. International standardization of diagnostic criteria for microvascular angina. Int J Cardiol. 2018;250:16-20.

103. Camici PG, Crea F. Coronary microvascular dysfunction. $N$ Engl J Med. 2007;356:830-840.

104. Ford TJ, Corcoran D, Sidik N, McEntegart M, Berry C. Coronary microvascular dysfunction: assessment of both structure and function. J Am Coll Cardiol. 2018;72:584-586.

105. Ford TJ, Stanley B, Good R, et al. Stratified medical therapy using invasive coronary function testing in angina: the CorMicA trial. J Am Coll Cardiol. 2018;72:2841-2855.

106. Recio-Mayoral A, Rimoldi OE, Camici PG, Kaski JC. Inflammation and microvascular dysfunction in cardiac syndrome $\mathrm{X}$ patients without conventional risk factors for coronary artery disease. JACC Cardiovasc Imaging. 2013;6: 660-667.

107. Sara JD, Widmer RJ, Matsuzawa Y, Lennon RJ, Lerman LO, Lerman A. Prevalence of coronary microvascular dysfunction among patients with chest pain and nonobstructive coronary artery disease. JACC Cardiovasc Interv. 2015;8: 1445-1453.

108. Taqueti VR, Shaw LJ, Cook NR, et al. Excess cardiovascular risk in women relative to men referred for coronary angiography is associated with severely impaired coronary flow reserve, not obstructive disease. Circulation. 2017;135: $566-577$.

109. Tavella R, Cutri N, Tucker G, Adams R, Spertus J, Beltrame JF. Natural history of patients with insignificant coronary artery disease. Eur Heart J Qual Care Clin Outcomes. 2016;2:117-124.

110. Jespersen L, Hvelplund A, Abildstrom SZ, et al. Stable angina pectoris with no obstructive coronary artery disease is associated with increased risks of major adverse cardiovascular events. Eur Heart J. 2012;33:734-744.

111. Ong P, Athanasiadis A, Borgulya G, Mahrholdt H, Kaski JC, Sechtem U. High prevalence of a pathological response to acetylcholine testing in patients with stable angina pectoris and unobstructed coronary arteries: the ACOVA Study (Abnormal COronary VAsomotion in patients with stable angina and unobstructed coronary arteries). J Am Coll Cardiol. 2012;59:655-662.

112. Herzog BA, Husmann L, Valenta I, et al. Long-term prognostic value of ${ }^{13} \mathrm{~N}-$ ammonia myocardial perfusion positron emission tomography added value of coronary flow reserve. J Am Coll Cardiol. 2009;54:150-156.

113. Murthy VL, Naya M, Taqueti VR, et al. Effects of sex on coronary microvascular dysfunction and cardiac outcomes. Circulation. 2014;129:2518-2527.

114. Bajaj NS, Osborne MT, Gupta A, et al. Coronary microvascular dysfunction and cardiovascular risk in obese patients. J Am Coll Cardiol. 2018;72:707-717.

115. Castagnoli H, Ferrantini C, Coppini R, et al. Role of quantitative myocardial positron emission tomography for risk stratification in patients with hypertrophic cardiomyopathy: a 2016 reappraisal. Eur J Nucl Med Mol Imaging. 2016;43:2413-2422.

116. Cecchi F, Olivotto I, Gistri R, Lorenzoni R, Chiriatti G, Camici PG. Coronary microvascular dysfunction and prognosis in hypertrophic cardiomyopathy. $N$ Engl J Med. 2003;349:1027-1035.

117. Lu DY, Yalcin H, Yalcin F, et al. Stress myocardial blood flow heterogeneity is a positron emission tomography biomarker of ventricular arrhythmias in patients with hypertrophic cardiomyopathy. Am J Cardiol. 2018;121:1081-1089.

118. Murthy VL, Naya M, Foster CR, et al. Improved cardiac risk assessment with noninvasive measures of coronary flow reserve. Circulation. 2011;124:22152224.

119. Olivotto I, Cecchi F, Gistri R, et al. Relevance of coronary microvascular flow impairment to long-term remodeling and systolic dysfunction in hypertrophic cardiomyopathy. J Am Coll Cardiol. 2006;47:1043-1048.

120. Osborne MT, Bajaj NS, Taqueti VR, et al. Coronary microvascular dysfunction identifies patients at high risk of adverse events across cardiometabolic diseases. J Am Coll Cardiol. 2017;70:2835-2837.

121. Marinescu MA, Loffler AI, Ouellette M, Smith L, Kramer CM, Bourque JM. Coronary microvascular dysfunction, microvascular angina, and treatment strategies. JACC Cardiovasc Imaging. 2015;8:210-220.

122. Gersh BJ, Maron BJ, Bonow RO, et al. 2011 ACCF/AHA guideline for the diagnosis and treatment of hypertrophic cardiomyopathy: executive summary: a 
report of the American College of Cardiology Foundation/American Heart Association Task Force on Practice Guidelines. Circulation. 2011;124:27612796.

123. Maron BJ, Rowin EJ, Udelson JE, Maron MS. Clinical spectrum and management of heart failure in hypertrophic cardiomyopathy. JACC Heart Fail. 2018;6:353-363

124. Sorajja P, Ommen SR, Nishimura RA, Gersh BJ, Berger PB, Tajik AJ. Adverse prognosis of patients with hypertrophic cardiomyopathy who have epicardial coronary artery disease. Circulation. 2003;108:2342-2348.

125. Geske JB, Ommen SR, Gersh BJ. Hypertrophic cardiomyopathy: clinical update. JACC Heart Fail. 2018;6:364-375.

126. Pavasini R, Camici PG, Crea F, et al. Anti-anginal drugs: systematic review and clinical implications. Int J Cardiol. 2019;283:55-63.

127. Rambarat CA, Elgendy IY, Handberg EM, et al. Late sodium channel blockade improves angina and myocardial perfusion in patients with severe coronary microvascular dysfunction: Women's Ischemia Syndrome Evaluation-Coronary Vascular Dysfunction ancillary study. Int J Cardiol. 2019;276:8-13.

128. Caobelli F, Haaf P, Chronis J, et al. Prognostic usefulness of cardiac stress test modalities in patients with type 2 diabetes mellitus who underwent myocardial perfusion scintigraphy (from the Basel Asymptomatic High-Risk Diabetics' Outcome Trial). Am J Cardiol. 2017;120:1098-1103.

129. Zellweger MJ, Haaf P, Maraun M, et al. Predictors and prognostic impact of silent coronary artery disease in asymptomatic high-risk patients with diabetes mellitus. Int J Cardiol. 2017;244:37-42.

130. Zellweger MJ, Maraun M, Osterhues HH, et al. Progression to overt or silent $\mathrm{CAD}$ in asymptomatic patients with diabetes mellitus at high coronary risk: main findings of the prospective multicenter BARDOT trial with a pilot randomized treatment substudy. JACC Cardiovasc Imaging. 2014;7:1001-1010.

131. Zellweger MJ, Hachamovitch R, Kang X, et al. Threshold, incidence, and predictors of prognostically high-risk silent ischemia in asymptomatic patients without prior diagnosis of coronary artery disease. J Nucl Cardiol. 2009;16: 193-200.

132. Young LH, Wackers FJ, Chyun DA, et al. Cardiac outcomes after screening for asymptomatic coronary artery disease in patients with type 2 diabetes: the DIAD study: a randomized controlled trial. JAMA. 2009;301:1547-1555.

133. Wackers FJ, Young LH, Inzucchi SE, et al. Detection of silent myocardial ischemia in asymptomatic diabetic subjects: the DIAD study. Diabetes Care. 2004;27:1954-1961.

134. Chow BJ, Dorbala S, Di Carli MF, et al. Prognostic value of PET myocardial perfusion imaging in obese patients. JACC Cardiovasc Imaging. 2014;7:278287.

135. AlBadri A, Bairey Merz CN, Johnson BD, et al. Impact of abnormal coronary reactivity on long-term clinical outcomes in women. J Am Coll Cardiol. 2019;73:684-693

136. Gulati M, Cooper-DeHoff RM, McClure C, et al. Adverse cardiovascular outcomes in women with nonobstructive coronary artery disease: a report from the Women's Ischemia Syndrome Evaluation Study and the St James Women Take Heart Project. Arch Intern Med. 2009;169:843-850.

137. Johnson BD, Shaw LJ, Buchthal SD, et al. Prognosis in women with myocardial ischemia in the absence of obstructive coronary disease: results from the $\mathrm{Na}$ tional Institutes of Health-National Heart, Lung, and Blood Institute-Sponsored Women's Ischemia Syndrome Evaluation (WISE). Circulation. 2004;109:29932999.

138. Johnson BD, Shaw LJ, Pepine CJ, et al. Persistent chest pain predicts cardiovascular events in women without obstructive coronary artery disease: results from the NIH-NHLBI-sponsored Women's Ischaemia Syndrome Evaluation (WISE) study. Eur Heart J. 2006;27:1408-1415.

139. Kay J, Dorbala S, Goyal A, et al. Influence of sex on risk stratification with stress myocardial perfusion Rb-82 positron emission tomography: results from the PET (Positron Emission Tomography) Prognosis Multicenter Registry. J Am Coll Cardiol. 2013;62:1866-1876.

140. Pepine CJ, Anderson RD, Sharaf BL, et al. Coronary microvascular reactivity to adenosine predicts adverse outcome in women evaluated for suspected ischemia results from the National Heart, Lung and Blood Institute WISE (Women's Ischemia Syndrome Evaluation) study. J Am Coll Cardiol. 2010;55:2825-2832.

141. Reis SE, Holubkov R, Conrad Smith AJ, et al. Coronary microvascular dysfunction is highly prevalent in women with chest pain in the absence of coronary artery disease: results from the NHLBI WISE study. Am Heart J. 2001; 141:735-741.

142. Shaw LJ, Merz CN, Pepine CJ, et al. The economic burden of angina in women with suspected ischemic heart disease: results from the National Institutes of Health-National Heart, Lung, and Blood Institute-sponsored Women's Ischemia Syndrome Evaluation. Circulation. 2006;114:894-904.
143. von Mering GO, Arant CB, Wessel TR, et al. Abnormal coronary vasomotion as a prognostic indicator of cardiovascular events in women: results from the National Heart, Lung, and Blood Institute-Sponsored Women's Ischemia Syndrome Evaluation (WISE). Circulation. 2004;109:722-725.

144. Sampson UK, Dorbala S, Limaye A, Kwong R, Di Carli MF. Diagnostic accuracy of rubidium-82 myocardial perfusion imaging with hybrid positron emission tomography/computed tomography in the detection of coronary artery disease. J Am Coll Cardiol. 2007;49:1052-1058.

145. Camici PG, Marraccini P, Lorenzoni R, et al. Coronary hemodynamics and myocardial metabolism in patients with syndrome $\mathrm{X}$ : response to pacing stress. J Am Coll Cardiol. 1991;17:1461-1470.

146. Cannon RO, Epstein SE. "Microvascular angina" as a cause of chest pain with angiographically normal coronary arteries. Am J Cardiol. 1988;61:1338-1343.

147. Chauhan A, Mullins PA, Taylor G, Petch MC, Schofield PM. Both endotheliumdependent and endothelium-independent function is impaired in patients with angina pectoris and normal coronary angiograms. Eur Heart J. 1997;18:60-68.

148. Panza JA, Laurienzo JM, Curiel RV, et al. Investigation of the mechanism of chest pain in patients with angiographically normal coronary arteries using transesophageal dobutamine stress echocardiography. J Am Coll Cardiol. 1997; 29:293-301.

149. Bairey Merz CN, Shaw LJ, Reis SE, et al. Insights from the NHLBI-Sponsored Women's Ischemia Syndrome Evaluation (WISE) Study: part II: gender differences in presentation, diagnosis, and outcome with regard to gender-based pathophysiology of atherosclerosis and macrovascular and microvascular coronary disease. J Am Coll Cardiol. 2006;47:S21-S29.

150. Kaski JC, Collins P, Nihoyannopoulos P, Maseri A, Poole-Wilson PA, Rosano GMC. Cardiac syndrome X: clinical characteristics and left ventricular function. J Am Coll Cardiol. 1995;25:807-814.

151. Quercioli A, Pataky Z, Montecucco F, et al. Coronary vasomotor control in obesity and morbid obesity: contrasting flow responses with endocannabinoids, leptin, and inflammation. JACC Cardiovasc Imaging. 2012;5:805-815.

152. Muzik O, Paridon SM, Singh TP, Morrow WR, Dayanikli F, Di Carli MF. Quantification of myocardial blood flow and flow reserve in children with a history of Kawasaki disease and normal coronary arteries using positron emission tomography. J Am Coll Cardiol. 1996;28:757-762.

153. Singh TP, Di Carli MF, Sullivan NM, Leonen MF, Morrow WR. Myocardial flow reserve in long-term survivors of repair of anomalous left coronary artery from pulmonary artery. J Am Coll Cardiol. 1998;31:437-443.

154. Singh TP, Humes RA, Muzik O, Kottamasu S, Karpawich PP, Di Carli MF. Myocardial flow reserve in patients with a systemic right ventricle after atrial switch repair. J Am Coll Cardiol. 2001;37:2120-2125.

155. Greenland P, Blaha MJ, Budoff MJ, Erbel R, Watson KE. Coronary calcium score and cardiovascular risk. J Am Coll Cardiol. 2018;72:434-447.

156. McClelland RL, Jorgensen NW, Budoff M, et al. 10-Year coronary heart disease risk prediction using coronary artery calcium and traditional risk factors: derivation in the MESA (Multi-Ethnic Study of Atherosclerosis) with validation in the HNR (Heinz Nixdorf Recall) Study and the DHS (Dallas Heart Study). J Am Coll Cardiol. 2015;66:1643-1653.

157. Blankstein R, Osborne M, Naya M, et al. Cardiac positron emission tomography enhances prognostic assessments of patients with suspected cardiac sarcoidosis. J Am Coll Cardiol. 2014;63:329-336.

158. Chareonthaitawee P, Beanlands RS, Chen W, et al. Joint SNMMI-ASNC expert consensus document on the role of ${ }^{18} \mathrm{~F}-\mathrm{FDG}$ PET/CT in cardiac sarcoid detection and therapy monitoring. J Nucl Cardiol. 2017;24:1741-1758.

159. Kruse MJ, Kovell L, Kasper EK, et al. Myocardial blood flow and inflammatory cardiac sarcoidosis. JACC Cardiovasc Imaging. 2017;10:157-167.

160. Mishra RK, Dorbala S, Logsetty G, et al. Quantitative relation between hemodynamic changes during intravenous adenosine infusion and the magnitude of coronary hyperemia: implications for myocardial perfusion imaging. J Am Coll Cardiol. 2005;45:553-558.

161. Feher A, Srivastava A, Quail MA, et al. Serial assessment of coronary flow reserve by rubidium- 82 positron emission tomography predicts mortality in heart transplant recipients. JACC Cardiovasc Imaging. 2020;13:109-120.

162. Aslam S, Khan M, Venetucci L, Arumugam P. Adenosine induced coronary vasospasm during Rubidium PET myocardial perfusion scan in a patient with Takayasu's Arteritis. J Nucl Cardiol. 2017;24:1114-1116.

163. Boden WE, O'Rourke RA, Teo KK, et al. Optimal medical therapy with or without PCI for stable coronary disease. N Engl J Med. 2007;356:1503-1516.

164. Schwartz RG, Pearson TA, Kalaria VG, et al. Prospective serial evaluation of myocardial perfusion and lipids during the first six months of pravastatin therapy. J Am Coll Cardiol. 2003;42:600-610.

165. Sdringola S, Nakagawa K, Nakagawa Y, et al. Combined intense lifestyle and pharmacologic lipid treatment further reduce coronary events and myocardial 
perfusion abnormalities compared with usual-care cholesterol-lowering drugs in coronary artery disease. J Am Coll Cardiol. 2003;41:263-272.

166. Shaw LJ, Berman DS, Maron DJ, et al. Optimal medical therapy with or without percutaneous coronary intervention to reduce ischemic burden: results from the Clinical Outcomes Utilizing Revascularization and Aggressive Drug Evaluation (COURAGE) trial nuclear substudy. Circulation. 2008;117:12831291.

167. Taqueti VR, Hachamovitch R, Murthy VL, et al. Global coronary flow reserve is associated with adverse cardiovascular events independently of luminal angiographic severity and modifies the effect of early revascularization. Circulation. 2015;131:19-27.

168. Mark DB, Shaw L, Harrell FE Jr, et al. Prognostic value of a treadmill exercise score in outpatients with suspected coronary artery disease. $N$ Engl J Med. 1991;325:849-853.

169. Hachamovitch R, Berman DS, Kiat H, et al. Exercise myocardial perfusion SPECT in patients without known coronary artery disease: incremental prognostic value and use in risk stratification. Circulation. 1996;93:905-914.

170. Gibbons RJ, Hodge DO, Berman DS, et al. Long-term outcome of patients with intermediate-risk exercise electrocardiograms who do not have myocardial perfusion defects on radionuclide imaging. Circulation. 1999;100:2140-2145.

171. Di Carli MF, Hachamovitch R. New technology for noninvasive evaluation of coronary artery disease. Circulation. 2007;115:1464-1480.

172. Nakazato R, Berman DS, Alexanderson E, Slomka P. Myocardial perfusion imaging with PET. Imaging Med. 2013;5:35-46.

173. Bourque JM, Charlton GT, Holland BH, Belyea CM, Watson DD, Beller GA. Prognosis in patients achieving $\geq 10$ METS on exercise stress testing: was SPECT imaging useful? J Nucl Cardiol. 2011;18:230-237.

174. Detrano R, Guerci AD, Carr JJ, et al. Coronary calcium as a predictor of coronary events in four racial or ethnic groups. $N$ Engl J Med. 2008;358: 1336-1345.

175. Kelkar AA, Schultz WM, Khosa F, et al. Long-term prognosis after coronary artery calcium scoring among low-intermediate risk women and men. Circ Cardiovasc Imaging. 2016;9:e003742.

176. Greenland P, Alpert JS, Beller GA, et al. 2010 ACCF/AHA guideline for assessment of cardiovascular risk in asymptomatic adults: a report of the American College of Cardiology Foundation/American Heart Association Task Force on Practice Guidelines. J Am Coll Cardiol. 2010;56:e50-e103.

177. Douglas PS, Hoffmann U, Patel MR, et al. Outcomes of anatomical versus functional testing for coronary artery disease. N Engl J Med. 2015;372:12911300.

178. Nissen SE, Cremer PC. Cardiac CT angiography: broken promises. American College of Cardiology website; May 15, 2015. https://www.acc.org/latest-incardiology/articles/2015/06/02/13/03/straight-talk-cardiac-ct-angiography-brokenpromises. Accessed October 3, 2019.

179. Shaw LJ, Hachamovitch R, Berman DS, et al. The economic consequences of available diagnostic and prognostic strategies for the evaluation of stable angina patients: an observational assessment of the value of precatheterization ischemia. J Am Coll Cardiol. 1999;33:661-669.

180. Shaw LJ, Hachamovitch R, Heller GV, et al. Noninvasive strategies for the estimation of cardiac risk in stable chest pain patients. Am J Cardiol. 2000; 86:1-7.

181. Iskandrian AS, Chae SC, Heo J, Stanberry CD, Wasserleben V, Cave V. Independent and incremental prognostic value of exercise single-photon emission computed tomographic (SPECT) thallium imaging in coronary artery disease. J Am Coll Cardiol. 1993;22:665-670.

182. Pancholy SB, Fattah AA, Kamal AM, Ghods M, Heo J, Iskandrian AS. Independent and incremental prognostic value of exercise thallium single-photon emission computed tomographic imaging in women. J Nucl Cardiol. 1995;2: $110-116$.
183. Taqueti VR, Dorbala S, Wolinsky D, et al. Myocardial perfusion imaging in women for the evaluation of stable ischemic heart disease-state-of-the-evidence and clinical recommendations. J Nucl Cardiol. 2017;24:1402-1426.

184. Weiser TG, Regenbogen SE, Thompson KD, et al. An estimation of the global volume of surgery: a modelling strategy based on available data. Lancet. 2008;372:139-144.

185. Smilowitz NR, Gupta N, Ramakrishna H, Guo Y, Berger JS, Bangalore S. Perioperative major adverse cardiovascular and cerebrovascular events associated with noncardiac surgery. JAMA Cardiol. 2017;2:181-187.

186. Puelacher C, Lurati Buse G, Seeberger D, et al. Perioperative myocardial injury after noncardiac surgery: incidence, mortality, and characterization. Circulation. 2018;137:1221-1232.

187. Beattie WS, Abdelnaem E, Wijeysundera DN, Buckley DN. A meta-analytic comparison of preoperative stress echocardiography and nuclear scintigraphy imaging. Anesth Analg. 2006;102:8-16.

188. Brown KA, Rowen M. Extent of jeopardized viable myocardium determined by myocardial perfusion imaging best predicts perioperative cardiac events in patients undergoing noncardiac surgery. J Am Coll Cardiol. 1993;21:325-330.

189. Bry JDL, Belkin M, O'Donnell TF, et al. An assessment of the positive predictive value and cost-effectiveness of dipyridamole myocardial scintigraphy in patients undergoing vascular surgery. J Vasc Surg. 1994;19:112-121.

190. Cohen MC. Perioperative and long-term prognostic value of dipyridamole Tc$99 \mathrm{~m}$ sestamibi myocardial tomography in patients evaluated for elective vascular surgery. J Nucl Cardiol. 2003;10:464-472.

191. Eagle KA, Coley CM, Newell JB, et al. Combining clinical and thallium data optimizes preoperative assessment of cardiac risk before major vascular surgery. Ann Intern Med. 1989;110:859-866.

192. Harafuji K, Chikamori T, Kawaguchi S, et al. Value of pharmacologic stress myocardial perfusion imaging for preoperative risk stratification for aortic surgery. Circ J. 2005;69:558-563.

193. Hendel RC, Whitfield SS, Villegas BJ, Cutler BS, Leppo JA. Prediction of late cardiac events by dipyridamole thallium imaging in patients undergoing elective vascular surgery. Am J Cardiol. 1992;70:1243-1249.

194. Lette J, Waters D, Cerino M, Picard M, Champagne P, Lapointe J. Preoperative coronary artery disease risk stratification based on dipyridamole imaging and a simple three-step, three-segment model for patients undergoing noncardiac vascular surgery or major general surgery. Am J Cardiol. 1992;69:1553-1558.

195. Marshall ES, Raichlen JS, Forman S, Heyrich GP, Keen WD, Weitz HH. Adenosine radionuclide perfusion imaging in the preoperative evaluation of patients undergoing peripheral vascular surgery. Am J Cardiol. 1995;76:817-821.

196. Stratmann HG, Younis LT, Wittry MD, Amato M, Miller DD. Dipyridamole technetium-99m sestamibi myocardial tomography in patients evaluated for elective vascular surgery: prognostic value for perioperative and late cardiac events. Am Heart J. 1996;131:923-929.

197. Younis LT, Aguirre F, Byers S, et al. Perioperative and long-term prognostic value of intravenous dipyridamole thallium scintigraphy in patients with peripheral vascular disease. Am Heart J. 1990;119:1287-1292.

198. Atkinson P, Chiu DY, Sharma R, et al. Predictive value of myocardial and coronary imaging in the long-term outcome of potential renal transplant recipients. Int J Cardiol. 2011;146:191-196.

199. Chew CG, Unger S, Shakib S. Value of myocardial perfusion imaging in renal transplant evaluation. Nephrology (Carlton). 2013;18:376-381.

200. Patel AD, Abo-Auda WS, Davis JM, et al. Prognostic value of myocardial perfusion imaging in predicting outcome after renal transplantation. Am J Cardiol. 2003;92:146-151

201. Singh N, Parikh S, Bhatt U, et al. Cardiac stress test as a risk-stratification tool for posttransplant cardiac outcomes in diabetic kidney transplant recipients. Transplantation. 2012;94:1224-1229. 